Check for updates

Cite this: Chem. Soc. Rev., 2021, 50, 2764

Received 28th September 2020

DOI: $10.1039 / \mathrm{d} 0 \operatorname{cs} 01160 f$

rsc.li/chem-soc-rev

\section{Two-dimensional conjugated metal-organic frameworks (2D c-MOFs): chemistry and function for MOFtronics}

\begin{abstract}
Mingchao Wang, (D) Renhao Dong* and Xinliang Feng (D)*
The 21st century has seen a reinvention of how modern electronics impact our daily lives; siliconelectronics and organic electronics are currently at the core of modern electronics. Recent advances have demonstrated that conductive metal-organic frameworks (MOFs), as another unique class of electronic materials, are emerging to provide additional possibility for multifunctional electronic devices that brings us "MOFtronics". Typically, two-dimensional conjugated MOFs (2D c-MOFs) are a novel class of layer-stacked MOFs with in-plane extended $\pi$-conjugation that exhibit unique properties such as intrinsic porosity, crystallinity, stability, and electrical conductivity as well as tailorable band gaps. Benefiting from their unique features and high conductivity, 2D c-MOFs have displayed great potential for multiple high-performance (opto)electronic, magnetic, and energy devices. In this review article, we introduce the chemical and synthetic methodologies of 2D c-MOFs, intrinsic influences on their electronic structures and charge transport properties, as well as multifunctional applications of this class of materials for MOFtronics and potential power sources for MOFtronics. We highlight the benefits and limitations of thus-far developed 2D c-MOFs from synthesis to function and offer our perspectives in regard to the challenges to be addressed.
\end{abstract}

Center for Advancing Electronics Dresden (cfaed) \& Faculty of Chemistry and Food Chemistry, Technische Universität Dresden, 01062 Dresden, Germany.

E-mail: xinliang:feng@tu-dresden.de, renhao.dong@tu-dresden.de

\section{Introduction}

Metal-organic frameworks (MOFs) represent crystalline coordination polymers, ${ }^{1-4}$ which are a class of porous materials with high surface area that were developed three decades ago.

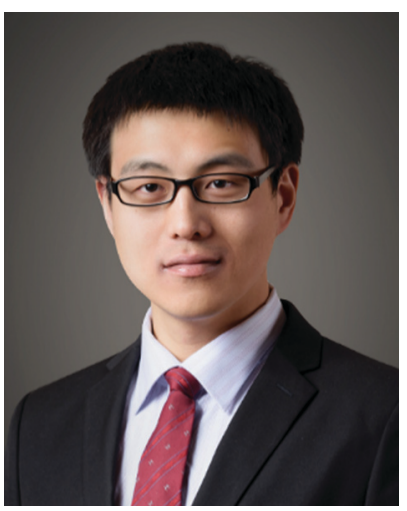

Mingchao Wang
Mingchao Wang is a PhD student under the supervision of Prof. Xinliang Feng in Technische Universität Dresden (TUD). He received his BSc from Shandong University in 2013 and MSc from TUD in 2016 in chemistry. His current research interests focus on the synthesis of novel twodimensional conjugated metalorganic frameworks and covalent organic frameworks as well as their applications in electronics and energy storage devices.

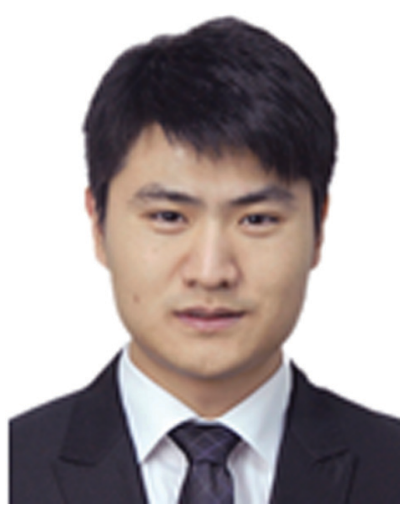

Renhao Dong
Renhao Dong is a TUD Young Investigator and leads an independent research group in Technische Universität Dresden. He received his Bachelor degree in chemistry in 2008 and then doctor degree in physical chemistry in 2013 from Shandong University. After that, he joined Max Planck Institute for Polymer Research and then TUD for postdoctoral research. In 01/ 2017, he was appointed as a group leader at TUD. His current scientific interests focus on chemistry of organic $2 D$ materials such as $2 D$ conjugated polymers, $2 D$ covalent organic frameworks, and $2 D$ metal-organic frameworks as well as their functions in electronics and energy storage/conversion. 
Chemical tunability in the porous structures ${ }^{5-7}$ allows various applications in gas storage, ${ }^{8,9}$ separation, ${ }^{10,11}$ catalysis $^{12,13}$ proton conduction, ${ }^{14}$ sensor, ${ }^{15,16}$ etc. The majority of traditional MOFs are electrical insulators which limit their utilization in multifunctional electronic devices. ${ }^{17,18}$ Twodimensional conjugated MOFs (2D c-MOFs) refer to layerstacked MOFs comprising ortho-substituted conjugated building blocks (e.g. benzene, triphenylene) and square-planar linkages with high in-plane conjugation and weak out-of-plane van der Waals interactions. The synthesis of 2D $c$-MOFs has been reported accompanied by intrinsic charge transport since $2012 .^{19}$ The extended $\pi$-conjugation in the $2 \mathrm{D}$ plane is capable of facilitating the delocalization of charge carriers within the network that is beneficial to high mobility and conductivity. ${ }^{20}$ Currently, over ten types of 2D c-MOFs have been developed based on conjugated ligands and linkages. ${ }^{21,22}$ Fig. 1 shows the timeline of the contribution to the synthesis of $2 \mathrm{D} c$-MOFs and the discovery of key properties from the community. Besides the preserved characteristics of traditional MOFs, such as tunable porosity, versatile structures and well-defined active sites, 2D $c$-MOFs have exhibited unique chemical and physical features such as high stability, electrochemical activity, photoactivity, tailorable band gaps, superior electrical conductivity, ferromagnetic ordering and topological state, ${ }^{23-27}$ thereby enabling 2D $c$-MOFs for broad applications in MOFtronics, such as (opto)electronics ${ }^{28-31}$ and spintronics, ${ }^{32-34}$ as well as the potential power sources for MOFtronics including battery ${ }^{35,36}$ and supercapacitor devices. ${ }^{37,38}$

This review article covers the chemistry and charge transport of 2D $c$-MOFs by consideration of structural diversity and structure-electronic property relationship based on the influence of molecular design (type and geometry of ligand, metal) and material structure (redox-activity in linkage, layer stacking and arrangement) on intrinsic charge transport properties.

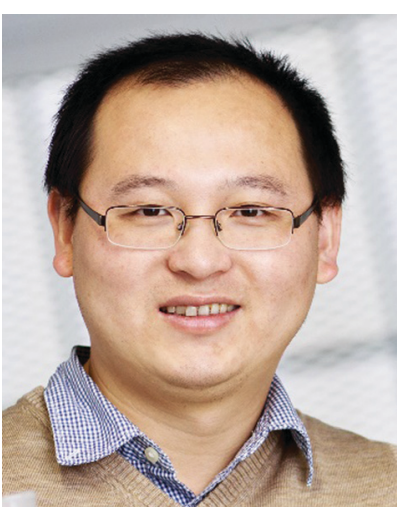

Xinliang Feng
Xinliang Feng is a full professor and has been the head of the Chair for Molecular Functional Materials at Technische Universität Dresden since 2014. His current scientific interests include organic synthetic methodology, organic synthesis, and supramolecular chemistry of $\pi$-conjugated systems, bottom-up synthesis of graphene and graphene nanoribbons, electrochemical exfoliation of $2 D$ crystals, $2 D$ polymers, and supramolecular polymers as well as $2 D$ carbon-rich conjugated polymers for optoelectronic applications, energy storage and conversion, and new energy devices and technologies. He has published more than 480 research articles which have attracted more than 52000 citations with an H-index of 109.
An overview of the synthetic methodologies including hydro-/ solvothermal methods and wet-interface-assisted strategies is further offered. Next, device fabrications and the applications of 2D $c$-MOFs for MOFtronics are presented covering (opto) electronics (e.g. field-effect transistors, superconductors, chemiresistors, photodetectors), spintronics, thermoelectrics, and energy storage devices with the discussion of their achievements and existing challenges. Critical directions for future research are proposed towards MOFtronics within the community.

\section{Chemical methodologies of 2D c-MOFs}

After the first 2D $c$-MOF reported by Yaghi group in $2012,{ }^{19}$ various ortho-substituted benzene- and triphenylene (TP)-based building blocks have been developed/employed for the formation of 2D $c$-MOFs, such as 1,2,3,4,5,6-hexahydroxybenzene (HHB), ${ }^{39}$ 1,2,3,4,5,6-hexaaminobenzene (HAB), ${ }^{40}$ 1,2,3,4,5,6benzenehexathiol (BHT, also denoted asHTB), ${ }^{41}$ 1,2,3,4,5,6benzenehexaselenol (HSeB), ${ }^{42}$ 2,3,6,7,10,11-hexahydroxytriphenylene (HHTP), ${ }^{19}$ 2,3,6,7,10,11-hexaaminotriphenylene (HATP), ${ }^{43,44}$ 2,3,6,7,10,11-triphenylenehexathiol (THT, also denoted as HTTP), ${ }^{45}$ 2,3,6,7,10,11-triphenylenehexaselenol (HSeTP). ${ }^{46}$ Larger conjugated monomers have been developed and explored for the synthesis of 2D c-MOFs as well (Fig. 2), e.g. 2,3,8,9,14,15-hexahydroxytrinaphthylene (HHTN), ${ }^{47}$ 2,3,7,8, 12,13-hexahydroxytruxene (HHTX), ${ }^{48} \operatorname{metal}(\mathrm{II}) \quad 2,3,8,9,14,16$ hexaaminohexaazatrinaphthylene HAHATNM$_{3}^{\prime}, \mathbf{M}^{\prime}=\mathrm{Cu}, \mathrm{Ni}$, Co), ${ }^{49}$ 1,2,3,4,5,6,7,8,9,10,11,12-perthiolated coronene (PTC), ${ }^{32}$ metal(II) 2,3,9,10,16,17,23,24-octahydroxyphthalocyanine $\left(\mathrm{M}^{\prime} \mathrm{Pc}(\mathrm{OH})_{8}, \quad \mathbf{M}^{\prime}=\mathrm{H}_{2}, \mathrm{Cu}, \mathrm{Fe}, \mathrm{Ni}, \mathrm{Zn}\right),{ }^{33,50-53} \operatorname{metal}(\mathrm{II})$ 2,3,9,10,16,17,23,24-octaaminophthalocyanine $\quad\left(\mathrm{M}^{\prime} \mathrm{Pc}\left(\mathrm{NH}_{2}\right)_{8}\right.$, $\left.\mathrm{M}^{\prime}=\mathrm{Cu}, \mathrm{Ni}\right){ }^{54,55} \operatorname{metal}(\mathrm{II}) 3,4,12,13,21,22,30,31$-octahydroxynaphthalocyanine $\left(\mathrm{M}^{\prime} \mathrm{NPc}(\mathrm{OH})_{8}, \mathrm{M}^{\prime}=\mathrm{Ni}\right) \cdot{ }^{51}$ Besides, a non-planar conjugated ligand, 2,3,6,7,10,11,14,15-octahydroxydibenzo[ $g, p]$ chrysene $\left(\mathrm{DBC}(\mathrm{OH})_{8}\right),{ }^{56}$ was included into the $2 \mathrm{D} c$-MOF family recently.

In fact, the ligands in most of the thus-far constructed $2 \mathrm{D}$ $c$-MOFs are partially oxidized to form charge-neutral frameworks, instead of the generally displayed structures which correspond to negatively charged linkages (e.g. $\mathrm{Cu}_{3}(\mathrm{HHTP})_{2}$, Fig. 10 in Section 4.1). ${ }^{19,23,25,40,55-58}$ Meanwhile, negatively charged 2D $c$-MOFs are also present in this emerging field, such as $\mathrm{Cu}_{3}(\mathrm{HHB})_{2}$ (Section 3.4). ${ }^{39,59}$ Among the reported 2D $c$-MOFs, three types of linkages containing chelating functional groups were usually adopted: metalbis(dioxolene) (abbreviated as $\mathbf{M O}_{4}$ ), metal-bis(diimine) $\left(\mathrm{MN}_{4}\right.$, also termed as metal-bis(iminobenzosemiquinoid)), and metal-bis(dithiolene) $\left(\mathrm{MS}_{4}\right)$ complexes $(\mathrm{M}=\mathrm{Fe}, \mathrm{Co}, \mathrm{Ni}$, $\mathrm{Cu}, \mathrm{Zn}, \mathrm{Pd}$, or $\mathrm{Pt}$ ). Due to the presence of both neutral and charged $\mathrm{MO}_{4}$-linked networks, the term of dioxolene instead of the normally applied semiquinoid is likely more reasonable..$^{25,60}$ In addition, metal-diimine-dithiolene $\left(\mathrm{MN}_{2} \mathrm{~S}_{2}\right)$ mixed linkage and metal-bis(diselenolene) $\left(\mathrm{MSe}_{4}\right)$ 

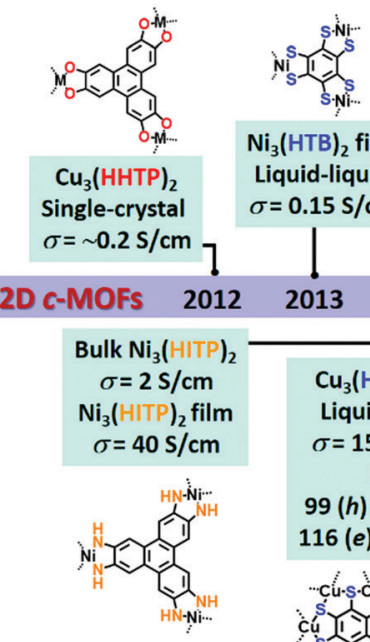

$\mathrm{Ni}_{3}(\mathrm{HTB})_{2}$ film

$\sigma=0.15 \mathrm{~S} / \mathrm{cm}$

$\left.\right|_{2013}$

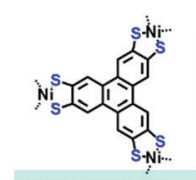

Bulk $\mathrm{Ni}_{3}(\mathrm{HITP})_{2}$

Thermoelectricity

$\mathrm{Ni}_{3}$ (HTTP) $)_{2}$ film

Single-layer

Gas-liquid (LB)

Water splitting

1
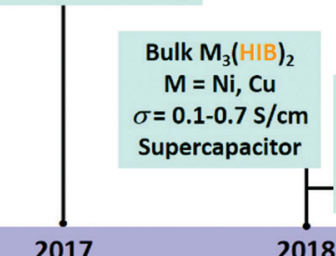

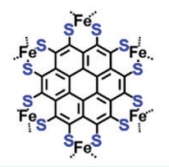

Bulk Fe (PTC) $_{3}$

$\sim 10 \mathrm{~s} / \mathrm{cm}$

Ferromagnetism

@ $\sim 15 \mathrm{~K}$

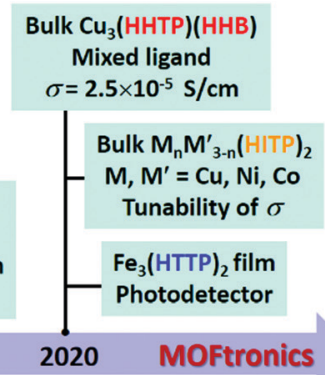

Bulk $\mathrm{Cu}_{3}$ (HHTP)(HHB)

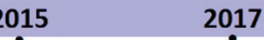

2019

2020 MOFtronics
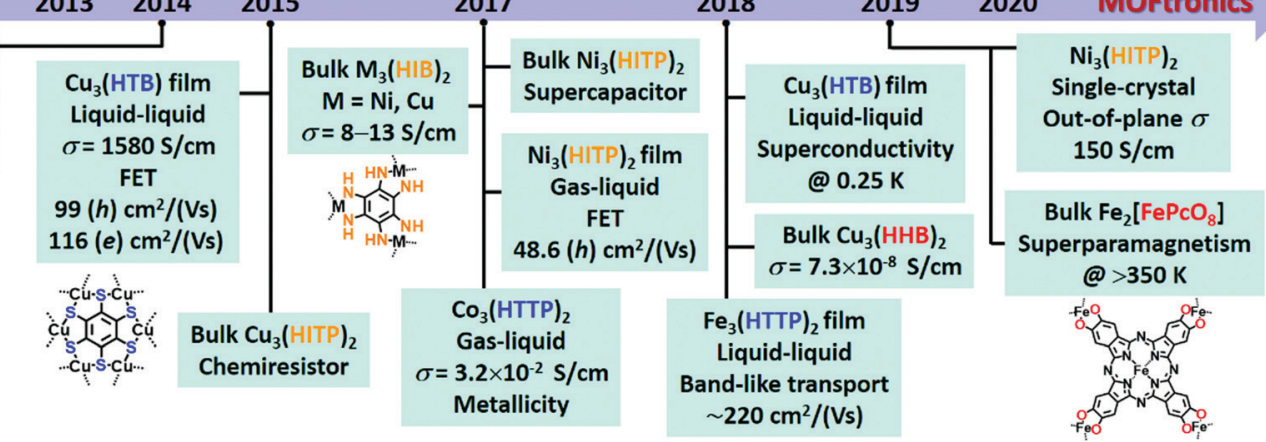

Fig. 1 Timeline in the development of 2D c-MOFs from synthesis to MOFtronics. The ligands containing $-\mathrm{OH}_{1}-\mathrm{NH}_{2}$, and $-\mathrm{SH}$ are marked in red, orange, and blue colour, respectively. The $\mathrm{Cu}_{3}(\mathrm{HHTP})(\mathrm{HHB}) 2 \mathrm{D} c-\mathrm{MOF}$ is also denoted as $\mathrm{Cu}_{3}(\mathrm{HHTP})(\mathrm{THQ})$ (THQ = tetraoxyquinone in $2 \mathrm{D} c-\mathrm{MOF}$ ). For 2D c-MOFs, HHTP represents 2,3,6,7,10,11-hexaoxytriphenylene instead of 2,3,6,7,10,11-hexahydroxytriphenylene for the monomer.

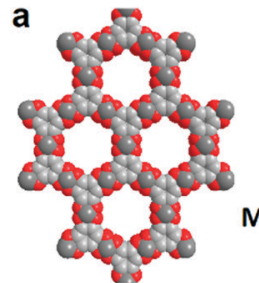

C
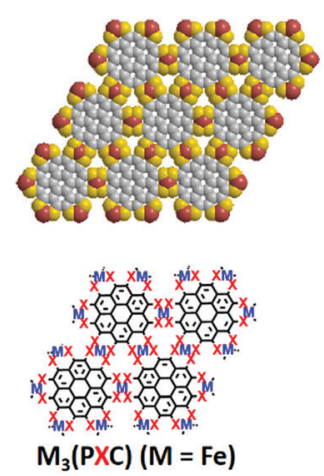

回

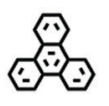

$\begin{array}{lll}M_{3}(H X B)_{2} & M_{3}(H X T P)_{2} & M_{3}(H X T N)_{2}\end{array}$ d

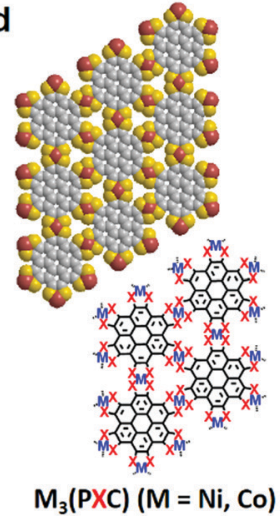

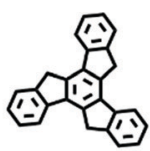

$M_{3}(\text { HXTX })_{2} \quad M_{3}\left(\text { HXHATNM }_{3}^{\prime}\right)_{2}$
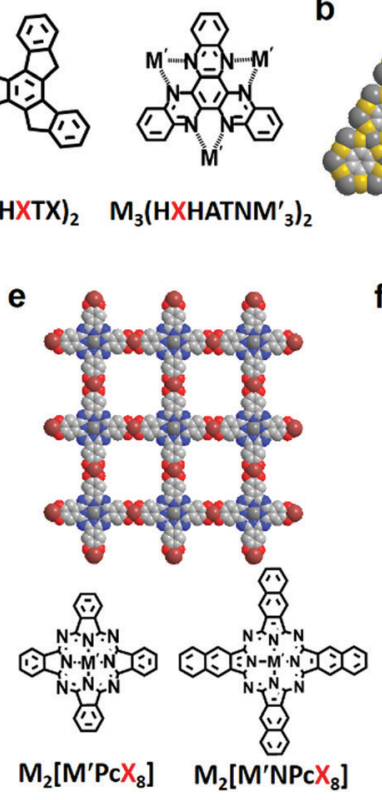

b

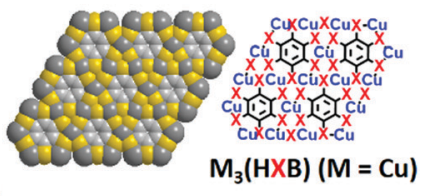

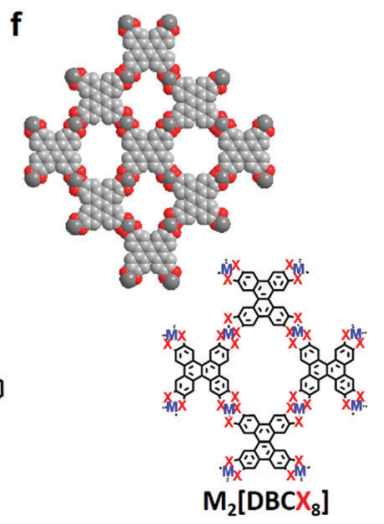

Fig. 2 Structure of various 2D c-MOFs. (a) 2D c-MOFs with honeycomb lattice. $M_{3}(H X B)_{2}, M_{3}(H X T P)_{2}, M_{3}(H X T N)_{2}, M_{3}(H X T X)_{2}, M_{3}\left(H X H A T N M{ }_{3}^{\prime}\right)_{2}$. $(b-d)$ Non-porous 2D c-MOFs. $M_{3}(H X B)\left(M=C u ; X=S\right.$, Se) and $M_{3}(P X C)(M=F e, N i, C u ; X=S)$. (e) $2 D$ c-MOFs with square lattice. $M_{2}\left[M^{\prime} P c X_{8}\right]$ $\left(M=F e, C o, N i, C u, Z n ; M^{\prime}=F e, N i, C u, Z n ; X=O, N H\right)$ and $M_{2}\left[M^{\prime} N P c X_{8}\right]\left(M=N i, C u ; M^{\prime}=N i ; X=O\right)$. (f) Non-planar 2D c-MOF. $M_{2}\left[D B C X_{8}\right]$ $(\mathrm{M}=\mathrm{Cu} ; \mathrm{X}=\mathrm{O})$.

linkage were also investigated in few reports. ${ }^{42,46,61-63}$ Among these various linkages, $\mathrm{Cu}$ and $\mathrm{Ni}$ represented the majority of metal nodes, while Fe and Co were widely utilized for the construction of $\mathrm{MS}_{4}$. By contrast, the utilization of $\mathrm{Zn}$, $\mathrm{Pd}$, and Pt remains very limited. ${ }^{53,64-67}$
Due to the increasing ligand library and the diverse compositions, a uniform description shall be helpful for the development of 2D $c$-MOF families. Based on the mostly accepted style, we label the benzene- and triphenylene-based 2D $c$-MOFs as $\mathbf{M}_{n}(\mathrm{HXL})_{m}$ (Fig. 2a), in which $\mathbf{M}$ and $\mathrm{L}$ represent the type of 
metal node and abbreviation of the building block (B for benzene, TP for triphenylene) respectively for a smallest repeating unit in their chemical structures, while $\mathrm{H}$ and $\mathrm{X}$ correspond to six substituent $\mathrm{X}$ in each ligand $(\mathrm{X}=\mathrm{O}, \mathrm{NH}, \mathrm{S}$, and Se). This can be also applied to trinaphthylene (TN)-, truxene (TX)- and hexaazatrinaphthylene (HATN)-based systems as $\mathbf{M}_{n}(\mathrm{HXTN})_{m}$, $\mathbf{M}_{n}(\mathrm{HXTX})_{m}$, and $\mathbf{M}_{n}\left(\mathrm{HXHATNM}_{3}\right)_{m}$ (Fig. 2a). Among all these systems, $n$ and $m$-the number of metal nodes and ligands-are confirmed to be 3 and 2 respectively in general, except for rare instances including $\mathrm{Ni}_{9}(\mathrm{HHTP})_{4}, \mathrm{Co}_{9}(\mathrm{HHTP})_{4} \quad$ (Fig. 10c), ${ }^{19}$ $\mathrm{Cu}_{3}$ (HTB), and $\mathrm{Cu}_{3}$ (HSeB) $\left(n=3, m=1\right.$, Fig. 2b). ${ }^{31,42,68}$ For a non-porous $2 \mathrm{D} c$-MOF based on the fully substituted coronene, ${ }^{32}$ $\mathrm{M}_{3}$ (PXC) is adopted (Fig. $2 \mathrm{c}$ and d), in which $\mathrm{P}$ and $\mathrm{C}$ represent full substitution in the ligand, and the abbreviation of coronene, respectively. However, the octa-substituted metallophthalocyanine $\left(\mathrm{M}^{\prime} \mathrm{Pc}\right)$ and dibenzo $[g, p]$ chrysene (DBC) are complicated systems. Instead of a scientific abbreviation for the ligands, the visualized one $\left(\mathrm{M}^{\prime} \mathrm{PcX}_{8}\right.$ for $\mathrm{M}^{\prime} \mathrm{Pc}{ }^{33,50-52,54,55} \mathrm{DBCX}_{8}$ for $\left.\mathrm{DBC}^{56}\right)$ is applied, thus leading to $\mathrm{M}_{2}\left[\mathrm{M}^{\prime} \mathrm{PcX}_{8}\right]$ (Fig. 2e) and $\mathrm{M}_{2}\left[\mathrm{DBCX}_{8}\right]$ (Fig. 2f) $2 \mathrm{D} c$ MOFs, respectively. This address is also applicable to the larger metallonaphthalocyanine ( $\mathrm{M}^{\prime} \mathrm{NPc}$ ) system (Fig. 2e). ${ }^{51}$ Notably, three-dimensional (3D) MOFs without layer-stacked structures could be also constructed based on planar ligands (e.g. HHTP, $\left.\mathrm{CoPc}(\mathrm{OH})_{8}\right)$ through metal-catecholate linkages. ${ }^{69-72}$ Therefore, the structure of 2D c-MOFs highly depends on the employed ligands, metal centers and synthetic conditions. ${ }^{73}$

\section{Chemical structure and electronic property relationship in 2D c-MOFs}

Conductivity $(\sigma)$ represents the transport of carriers of electrons $(e)$ and holes $(h)$, which can be quantified with mobility $(\mu)$ and density $(n): \sigma=e\left(n_{e} \mu_{e}+n_{h} \mu_{h}\right)$. Free charge carriers in 2D $c$-MOFs can be generated through thermal/photo-population or charge carrier injection through redox reactions. ${ }^{23,74,75}$ In this context, both the metal nodes (Frontier orbitals) and the ligands can serve as the sources. The potential charge transport pathways in 2D $c$-MOFs were ascribed as: through-bond, extended conjugation, through-space, and hopping. ${ }^{25}$ Particularly, through-bond or extended conjugation pathways in the 2D plane and throughspace channel (strong $\pi-\pi$ stacking and weak metal-metal interactions) dominate the intrinsic conductivity of $2 \mathrm{D} c$-MOFs, while hopping stems from the charge carrier migration through grain boundaries, defects, etc. Many inspiring review articles have provided in-depth understanding on the above transport mechanism as well as the description of current characterization methods toward conductivity measurement, such as twoprobe and four-probe (linear four-probe or van der Pauw) configurations. ${ }^{17,20,22-25,60,76}$ Table 1 lists the currently reported 2D $c$-MOFs and the related studies on charge transport, including the sample form, characterization method, conductivity and mobility. Hereby, we would like to discuss the influence of molecular design (type and geometry of ligand, metal) and material structure (redox-activity in linkage, layer stacking and arrangement) as well as crystallinity on the charge transport properties for thus-far reported materials.

\subsection{Effect of ligand type}

The current ligands utilized for the construction of 2D $c$-MOFs have been mainly based on the planar conjugated building blocks with symmetrical functional groups of $-\mathrm{OH},-\mathrm{NH}_{2}$, and -SH. Varying the functional groups results in increased bond strength between metals and linkers from $\mathrm{MO}_{4}$ to $\mathrm{MN}_{4}$ to $\mathrm{MS}_{4},{ }^{25}$ thus leading to the apparent enhancement of the inplane charge transport along the 2D network. Density functional theory (DFT) calculations indicated that the linker substitution in $\mathrm{MX}_{4}(\mathrm{X}=\mathrm{O}, \mathrm{NH}$, and $\mathrm{S})$ also affects the dynamic stability of the frameworks; $\mathrm{Zn}_{3}(\mathrm{HTB})$ is more stable than $\mathrm{Zn}_{3}$ (HSeB) whilst $\mathrm{Zn}_{3}(\mathrm{HHB})$ is the most unstable one. ${ }^{77}$ In addition, energy matching of the $\mathrm{d}$ orbitals of different transition metals with the $\pi$-conjugated ligands generally generates the difference in $\pi-d$ hybridization, hence tuning the delocalization of carriers in the $2 \mathrm{D}$ plane to a different extent (Section 3.3). Among these 2D $c$-MOFs, $\mathrm{MO}_{4}$-linked ones exhibited apparently inferior charge transport to the related $2 \mathrm{D}$ $c$-MOFs linked by the $\mathrm{MN}_{4}$ coordination complex. For instance, charge-neutral $\mathrm{MO}_{4}$-linked $\mathrm{Cu}_{3}(\mathrm{HHTP})_{2}$ could display broad conductivity values in the range of $10^{-4}$ to $1 \mathrm{~S} \mathrm{~cm}^{-1}$ measured by 2-/4-probe measurement in their pellet form at room temperature. By contrast, the pellet samples of $\mathrm{MN}_{4}$-linked $\mathrm{Cu}_{3}(\mathrm{HITP})_{2}$ could exhibit higher conductivity values in the range of 0.2-0.75 $\mathrm{S} \mathrm{cm}^{-1}$. Regarding Pc-based $\mathrm{M}_{2}\left[\mathrm{M}^{\prime} \mathrm{PcX}_{8}\right] 2 \mathrm{D}$ $c$-MOFs, the $\mathrm{MN}_{4}$-linked samples also showed superior conductivity than the $\mathrm{MO}_{4}$-linked ones. The $\mathrm{MS}_{4}$ complex presents the highest $\pi-d$ hybridization among these linkages, and therefore thus-far reported $\mathrm{MS}_{4}$-linked 2D $c$-MOFs have been demonstrated to exhibit excellent conductivity and mobility. For example, the $\mathrm{Ni}_{3}(\mathrm{HTB})_{2}$ film showed a conductivity of $\sim 160 \mathrm{~S} \mathrm{~cm}^{-1}$ and the non-porous $\mathrm{Cu}_{3}$ (HTB) film could even exhibit a conductivity as high as $\sim 2500 \mathrm{~S} \mathrm{~cm}^{-1}$ (shown in Table 1). Besides the conductivity, another typical $\mathrm{MS}_{4}$-linked sample, $\mathrm{Fe}_{3}(\mathrm{HTTP})_{2}$, was observed to present a notable band-like transport with record high charge mobility $>200 \mathrm{~cm}^{2} \mathrm{~V}^{-1} \mathrm{~s}^{-1}$ amongst the reported 2D $c$-MOFs due to the high $\pi-\mathrm{d}$ conjugation. ${ }^{78}$ The $\mathrm{Co}_{3}(\mathrm{HTTP})_{2} 2 \mathrm{D} \quad c$-MOF was recorded to possess a negative temperature-resistivity relationship in the high temperature region and a positive trend at low temperature for semiconducting and metallic behavior, respectively. ${ }^{79,80}$ Recently, $\mathrm{MSe}_{4}$-linked bulk 2D $c$-MOFs have been developed and displayed lower conductivity $\left(\right.$ e.g. $\mathrm{Cu}_{3}(\mathrm{H}-$ $\mathrm{SeB}): 110 \mathrm{~S} \mathrm{~cm}^{-1}, \mathrm{Co}_{3}(\mathrm{HSeTP})_{2}: 10^{-6} \mathrm{~S} \mathrm{~cm}^{-1}$ ) than those of $\mathrm{MS}_{4^{-}}$ linked bulk 2D $c$-MOFs (Table 1 ). ${ }^{42,46}$

Varying the functional groups will also vary the coordination bond length between metal nodes and linkers in the $\mathrm{MX}_{4}$ linkages due to the difference of the atom radius of the $\mathrm{O}, \mathrm{N}, \mathrm{S}$, and $\mathrm{Se}(\mathrm{Se}>\mathrm{S}>\mathrm{O}>\mathrm{N}$ ), which plays also an important role in the charge transport and other physical properties. For instance, for some selected square-planar Ni-coordinated complexes (Fig. 3A-C), the bond lengths are 1.866, 1.822-1.826, and 2.132-2.170 A for Ni-O, Ni-N, and Ni-S bonds, respectively. ${ }^{81-83}$ 
Table 1 Summary of the literature reported electrical conductivities and mobilities for 2D c-MOFs

\begin{tabular}{|c|c|c|c|c|c|c|}
\hline 2D $c-\mathrm{MOF}$ & Building block & Type & Method & $\sigma^{a}\left(\mathrm{~S} \mathrm{~cm}^{-1}\right)$ & $\mu\left(\mathrm{cm}^{2} \mathrm{~V}^{-1} \mathrm{~s}^{-1}\right)$ & Ref. \\
\hline \multicolumn{7}{|c|}{ Metal-bis(dioxolene) linked 2D $c$-MOFs } \\
\hline \multirow[t]{3}{*}{$\mathrm{Cu}_{3}(\mathrm{HHB})_{2}$} & B & Pellet & $\operatorname{vdP} P^{b}$ & $7.8 \times 10^{-8}$ & & 39 \\
\hline & & Pellet & - & $2.2 \times 10^{-9}$ & & 87 \\
\hline & & Pellet $^{c}$ & vdP & $1.5 \times 10^{-7}$ & $2.4\left(\right.$ Hall,$\left.h^{d}\right)$ & 88 \\
\hline \multirow[t]{13}{*}{$\mathrm{Cu}_{3}(\mathrm{HHTP})_{2}$} & $\mathrm{TP}$ & Pellet & 2-Probe & $1.0 \times 10^{-2}$ & & 89 \\
\hline & & Pellet & 2-Probe & $3.0 \times 10^{-3}$ & & 90 \\
\hline & & Pellet & 2-Probe & $2.0 \times 10^{-3}$ & & 91 \\
\hline & & Pellet & 4-Probe & $2.0 \times 10^{-2}$ & & 92 \\
\hline & & Pellet & vdP & $4.5 \times 10^{-2}$ & & 93 \\
\hline & & Pellet & vdP & $2.7 \times 10^{-2}$ & & 94 \\
\hline & & Pellet & - & $0.09-0.1$ & & 95 \\
\hline & & Film & 2-Probe & $2.0 \times 10^{-2}$ & & 96 \\
\hline & & Film & 4-Probe & 0.29 & & 97 \\
\hline & & Film & - & $10^{-4}$ & & 98 \\
\hline & & Nanorod $^{e}$ & 4-Probe & 0.21 & & 19 \\
\hline & & Nanorod $^{e}$ & 4-Probe & 1.5 & & 95 \\
\hline & & Nanoflake $^{e}$ & 2-Probe & 0.5 & & 95 \\
\hline \multirow{7}{*}{$\mathrm{Ni}_{9}(\mathrm{HHTP})_{4}$} & $\mathrm{TP}$ & Pellet & 2-Probe & $6.0 \times 10^{-3}$ & & 99 \\
\hline & & Pellet & 4-Probe & $1.0 \times 10^{-2}$ & & 100 \\
\hline & & Pellet & 4-Probe & 0.1 & & 92 \\
\hline & & Pellet & 4-Probe & 0.26 & & 101 \\
\hline & & Pellet & vdP & $6.8 \times 10^{-3}$ & & 94 \\
\hline & & Film & vdP & $1.1 \times 10^{-3}$ & & 94 \\
\hline & & Hybrid & 2-Probe & $1.6 \times 10^{-4}$ & & 102 \\
\hline \multirow[t]{4}{*}{$\mathrm{Co}_{9}(\mathrm{HHTP})_{4}$} & $\mathrm{TP}$ & Pellet & 2-Probe & $2.0 \times 10^{-3}$ & & 99 \\
\hline & & Pellet & 4-Probe & $2.7 \times 10^{-6}$ & & 92 \\
\hline & & Pellet & $\mathrm{vdP}$ & $3.2 \times 10^{-2}$ & & 94 \\
\hline & & Film & vdP & $3.3 \times 10^{-3}$ & & 94 \\
\hline $\mathrm{Cu}_{3}(\mathrm{HHTP})(\mathrm{HHB})$ & $\mathrm{TP} / \mathrm{B}$ & Pellet & 2-Probe & $2.5 \times 10^{-5}$ & & 59 \\
\hline $\mathrm{Cu}_{3}(\mathrm{HHTN})_{2}$ & $\mathrm{TN}$ & Pellet & 2-Probe & $9.6 \times 10^{-10}$ & & 47 \\
\hline $\mathrm{Cu}_{3}(\mathrm{HHTX})_{2}$ & $\mathrm{TX}$ & Pellet & 2-Probe & $8.3 \times 10^{-4}$ & & 48 \\
\hline $\mathrm{Cu}_{2}\left[\mathrm{DBCO}_{8}\right]$ & DBC & Pellet & - & $1 \times 10^{-2}$ & & 56 \\
\hline \multirow[t]{2}{*}{$\mathrm{Cu}_{2}\left[\mathrm{CuPcO}_{8}\right]$} & $\mathrm{Pc}$ & Pellet & 2-Probe & $9.4 \times 10^{-8}$ & & 50 \\
\hline & & Pellet & 2-Probe & $1.6 \times 10^{-6}$ & & 50 \\
\hline $\mathrm{Cu}_{2}\left[\mathrm{NiPcO}_{8}\right]$ & $\mathrm{Pc}$ & Pellet & 4-Probe & $1.4 \times 10^{-2}$ & & 51 \\
\hline $\mathrm{Ni}_{2}\left[\mathrm{NiPcO}_{8}\right]$ & Pc & Pellet & 4-Probe & $7.2 \times 10^{-4}$ & & 51 \\
\hline \multirow{2}{*}{$\mathrm{Fe}_{2}\left[\mathrm{FePcO}_{8}\right]$} & $\mathrm{Pc}$ & Pellet & vdP & $\sim 1 \times 10^{-5}$ & $\sim 0.1(\mathrm{Hall}, h)$ & 33 \\
\hline & & Pellet & - & - & 15 (TRTS) & 33 \\
\hline $\mathrm{Cu}_{2}\left[\mathrm{NiNPcO}_{8}\right]$ & $\mathrm{NPc}$ & Pellet & 4-Probe & $3.1 \times 10^{-2}$ & & 51 \\
\hline $\mathrm{Ni}_{2}\left[\mathrm{NiNPcO}_{8}\right]$ & $\mathrm{NPc}$ & Pellet & 4-Probe & $1.8 \times 10^{-2}$ & & 51 \\
\hline \multicolumn{7}{|c|}{ Metal-bis(diimine) linked 2D $c$-MOFs } \\
\hline \multirow[t]{2}{*}{$\mathrm{Cu}_{3}(\mathrm{HIB})_{2}$} & B & Pellet & 4-Probe & 0.1 & & 38 \\
\hline & & Pellet & vdP & 13 & & 40 \\
\hline \multirow[t]{2}{*}{$\mathrm{Ni}_{3}(\mathrm{HIB})_{2}$} & $\mathrm{~B}$ & Pellet & 4-Probe & 0.7 & & 38 \\
\hline & & Pellet & vdP & 8 & & 40 \\
\hline $\mathrm{Co}_{3}(\mathrm{HIB})_{2}$ & B & Pellet & 4-Probe & $0.1-1.57$ & & 35 \\
\hline \multirow[t]{2}{*}{$\mathrm{Cu}_{3}(\mathrm{HITP})_{2}$} & $\mathrm{TP}$ & Pellet & 2-Probe & 0.2 & & 28 \\
\hline & & Pellet & 4-Probe & 0.75 & & 58 \\
\hline $\mathrm{Ni}_{3}(\mathrm{HITP})_{2}$ & $\mathrm{TP}$ & Pellet & 2-Probe & 2 & & 57 \\
\hline & & Pellet & 4-Probe & 39 & & 101 \\
\hline & & Pellet & 4-Probe & 1.896 & & 103 \\
\hline & & Pellet & - & 0.5 & & 104 \\
\hline & & Pellet & vdP & 50 & & 37 \\
\hline & & Pellet & 4-Probe & 55.4 & & 58 \\
\hline & & Pellet & vdP & 58.8 & & 105 \\
\hline & & Film & - & 37.2 & & 104 \\
\hline & & Film & 2-Probe & $8.5 \times 10^{-3}$ & & 106 \\
\hline & & Film & 2-Probe & 0.23 & & 106 \\
\hline & & Film & vdP & 40 & & 57 \\
\hline & & Hybrid & 2-Probe & $2.6 \times 10^{-3}$ & & 102 \\
\hline & & Nanorod $^{e}$ & 4-Probe & 150 & & 95 \\
\hline & & Film & FET $^{g}$ & - & $48.6(h)$ & 29 \\
\hline & & Film & FET $^{g}$ & - & $45.4(h)$ & 107 \\
\hline $\mathrm{Co}_{3}(\mathrm{HITP})_{2}$ & $\mathrm{TP}$ & Pellet & 4-Probe & $8 \times 10^{-4}$ & & 103 \\
\hline & & Pellet & 4-Probe & 0.024 & & 58 \\
\hline $\mathrm{Co}_{\mathrm{a}} \mathrm{Cu}_{\mathrm{b}}(\mathrm{HITP})_{2}^{f}$ & $\mathrm{TP}$ & Pellet & 4-Probe & $5.8 \times 10^{-3}$ & & 58 \\
\hline $\left.\mathrm{Ni}_{3}(\mathrm{HXHATNNi})_{2}\right)_{2}$ & HATN & Pellet & 4-Probe & 2 & & \\
\hline $\mathrm{Ni}_{2}\left[\mathrm{NiPc}(\mathrm{NH})_{8}\right]$ & $\mathrm{Pc}$ & Film & 4-Probe & 0.2 & & 54 \\
\hline $\mathrm{Ni}_{2}\left[\mathrm{CuPc}(\mathrm{NH})_{8}\right]$ & $\mathrm{Pc}$ & Pellet $^{c}$ & vdP & $\sim 1 \times 10^{-4}$ & $1.6($ Hall, $h)$ & 55 \\
\hline
\end{tabular}


Table 1 (continued)

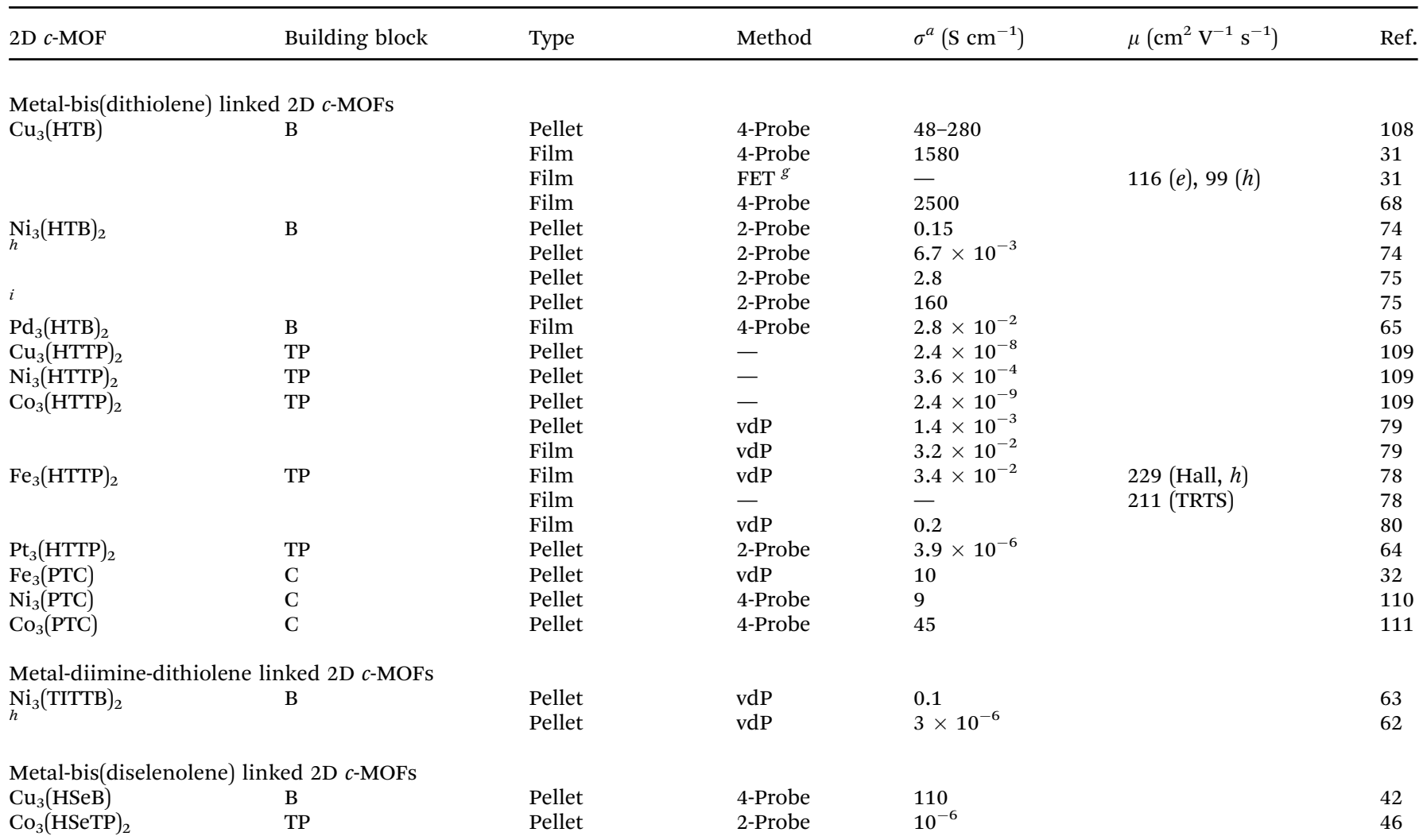

${ }^{a}$ Electrical conductivity at room temperature. ${ }^{b}$ Van der Pauw. ${ }^{c}$ Nanosheets. ${ }^{d}$ Hall effect. ${ }^{e}$ Device based on single-crystal. ${ }^{f} a=2.47, b=0.53$. ${ }^{g}$ Field-effect transistor. ${ }^{h}$ Reduced 2D $c$-MOF with charge state of $-1{ }^{i}$ Oxidized 2D $c$-MOF with charge state of 0 .

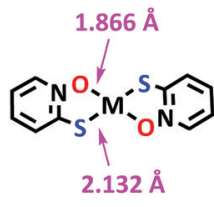

A

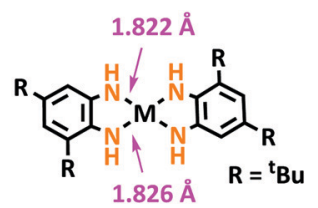

B

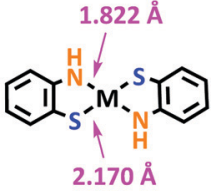

C
Metal-linker bond length

Fig. 3 The bond length of metal-linkers $(\mathrm{O}, \mathrm{NH}, \mathrm{S})$ in the $M \mathrm{X}_{4}$ linkage. Selected structures of $A,{ }^{75} B,{ }^{74}$ and $C^{76}$ refer to reported metal-complex small molecules.

While for $2 \mathrm{D} c$-MOFs, $\mathrm{Ni}_{3}(\mathrm{HITP})_{2}$ and $\mathrm{Ni}_{3}(\mathrm{HTTP})_{2}$, powder X-ray diffraction (PXRD) indicated prominent (100) peak $(\mathrm{Cu}-\mathrm{K} \alpha$ radiation) with $2 \theta=4.7^{\circ}(1.88 \mathrm{~nm})$ and $4.5^{\circ}$ (1.96 nm) respectively, indicative of larger lattice constant of $\mathrm{Ni}_{3}(\mathrm{HTTP})_{2}$ than $\mathrm{Ni}_{3}(\mathrm{HITP})_{2}$ due to the presence of its longer Ni-S bond. ${ }^{29,45,57}$ A similar phenomenon was revealed by the DFT calculations: $\sim 0.1 \mathrm{~nm}$ smaller lattice constant of $\mathrm{Mn}_{3}(\mathrm{HIB})_{2}$ than $\mathrm{Mn}_{3}(\mathrm{HTB})_{2} \cdot{ }^{84,85}$ In a monolayer of $\mathrm{Mn}_{3}(\mathrm{HIB})_{2}$, the shorter distance between $\mathrm{Mn}^{2+}$ ions is expected to enhance the magnetic coupling; compared with $\mathrm{MS}_{4}, \mathrm{~N}$ atoms in the $\mathrm{MN}_{4}$ linkage are more effective at mediating the magnetic couplings. As a result, the monolayer of $\mathrm{Mn}_{3}(\mathrm{HIB})_{2}$ is predicted to possess a transition temperature for ferromagnetic coupling at $\sim 450 \mathrm{~K}$, which considerably surpasses that of $212 \mathrm{~K}$ for a single-layer of $\mathrm{Mn}_{3}(\mathrm{HTB})_{2} \cdot{ }^{84}$ Nevertheless, it remains not sufficient to quantitatively compare the influence of the $\mathrm{MX}_{4}$ linkages on conductivity, due to the few examples and the heterogeneous sample morphologies, potential doping, as well as the lack of unique features of chemical composition and structural geometry for individual samples.

\subsection{Effect of ligand geometry}

Ligand geometry and symmetry are of key significance for the construction of regular frameworks of 2D c-MOFs. Currently, three types of networks have been developed for 2D c-MOFs, including hexagonal, square, and honeycomb lattices with polygons of triangles, squares, and hexagons, respectively (Fig. 4). For the construction of hexagonal, square, or honeycomb frameworks, symmetric ligands with C6 (e.g. PTC), C4 (e.g. $\mathrm{M}^{\prime} \mathrm{PcX} \mathrm{X}_{8}$ ), or $\mathrm{C} 3$ (e.g. HXTP) geometry respectively are required. Importantly, the resulting framework geometry is relevant to the electronic structure of the achieved 2D $c$-MOF. For example, the hexagonal and square geometries correlate with a structure, where only one type of vertex exists in each unit cell with six neighboring triangles or four neighboring squares (Fig. 4a and b, unit cells are marked red in the middle figures). DFT calculations indicated that only one type of band is presented in the band structures for both geometries. ${ }^{86} \mathrm{On}$ the other hand, each unit cell contains two vertices in different environments in a honeycomb network (Fig. 4c). As a result, 
a
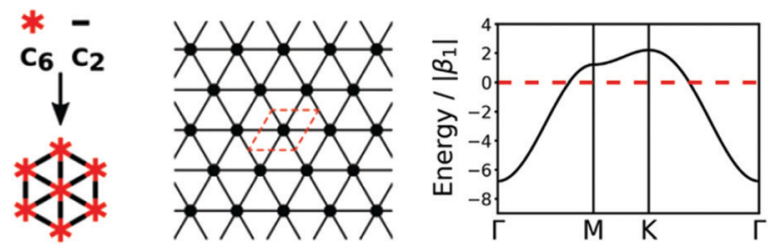

b
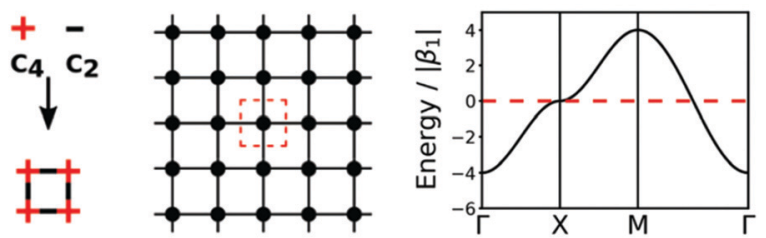

C
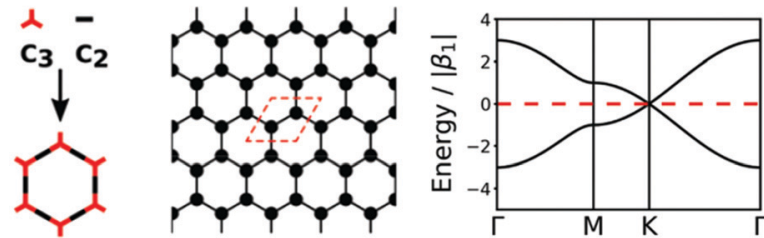

Fig. 4 Topological diagrams of the networks and 2D representations with different geometries (unit cells are marked in red) as well as corresponding band structures. (a) Hexagonal. (b) Square. (c) Honeycomb. C2 monomer refers to metal ions here ref. 86 . Copyright 2020, the Royal Society of Chemistry.

two bands appear in the energy band diagram forming the Dirac point.

Though a dual-porous kagome network (Fig. 5a, left) is not presented among thus-far reported $2 \mathrm{D} c$-MOFs, the metal atoms in the honeycomb frameworks (e.g. $\left.\mathrm{M}_{3}(\mathrm{HXB})_{2}, \mathrm{M}_{3}(\mathrm{HXTP})_{2}\right)$ are arranged in the kagome lattice (Fig. 5a, middle), which exhibits a band structure including a Dirac point and a flat band above the Dirac bands (Fig. 5a, right). ${ }^{86}$ The framework with extended $\pi$-conjugation in a kagome lattice is predicted to exhibit nontrivial physical properties. For instance, first-principles calculations indicated that a single-layer of $\mathrm{Ni}_{3}(\mathrm{HTB})_{2}$ possesses the typical kagome bands: a flat band above two Dirac bands (Fig. 5b and c). The local density of state of a semi-infinite $\mathrm{Ni}_{3}(\mathrm{HTB})_{2}$ framework for spin-up and spin-down components indicated nontrivial topological edge states (Fig. 5d and e), indicative of the possible existence of an organic topological insulator. ${ }^{112}$ Besides, a monolayer of $\mathrm{Ni}_{3}(\mathrm{HITP})_{2}$ (Fig. 8b) or $\mathrm{Cu}_{3}(\mathrm{HITP})_{2}$ also exhibited Dirac bands near the Fermi level. ${ }^{113}$

In addition to the type and geometry of the ligand, the ligand size (e.g. benzene, triphenylene, trinaphthylene), which correlates with diverse densities of building blocks and linkages as well as the $\pi$-conjugation degree in the network, is also an important parameter for the electronic structures. However, accompanied by other factors, such as the lattice, layer distance, porous density, etc., the influences of ligand size remain elusive. Besides, the control of $\pi$-conjugation degree in the building blocks (e.g. benzene, triphenylene, trinaphthylene, coronene, phthalocyanine) is expected to efficiently vary the conductivity and mobility of $2 \mathrm{D} c$-MOFs in both in-plane and cross-plane directions. Nonetheless, because of the differences in the geometry and size of the ligand, a proper relationship between $\pi$-conjugation and conductivity has been so far elusive.
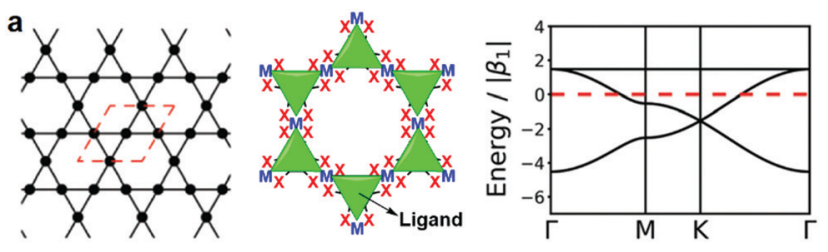

b
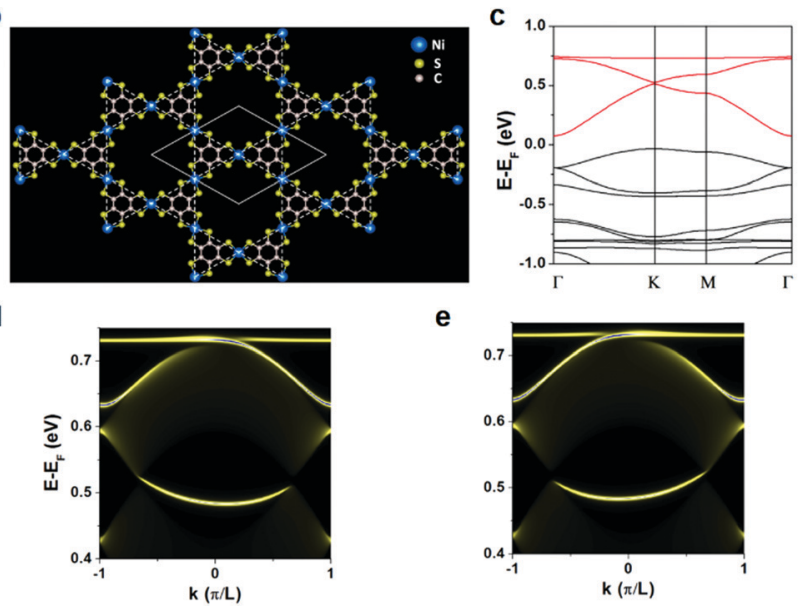

Fig. 5 Kagome lattice in 2D c-MOFs. (a) Topological diagram of the kagome network (left), the kagome lattice of metal atoms in a honeycomb network (middle), and the band structure for a kagome network (right). ${ }^{86}$ Copyright 2020, the Royal Society of Chemistry. (b) Kagome lattice of the $\mathrm{Ni}_{3}(\mathrm{HTB})_{2}$ monolayer. The dashed lines outline the kagome lattice. (c) The band structure of the $\mathrm{Ni}_{3}(\mathrm{HTB})_{2}$ monolayer. ( $d$ and e) The semi-infinite edge states of the spin-up and spin-down components for the $\mathrm{Ni}_{3}(\mathrm{HTB})_{2}$ monolayer, respectively. Reproduced with permission ref. 112. Copyright 2013, American Chemical Society.

\subsection{Effect of metals}

The metal centers have been demonstrated to play an important role in the electronic properties of the 2D c-MOFs. It is already known that the conductive 3D MOFs with mixed valence $\mathrm{Fe}$ atoms $\left(\mathrm{Fe}^{2 / 3+}\right)$ show superior electrical conductivity to the pure $\mathrm{Fe}^{2+}$-bridged ones. ${ }^{114-117}$ For instance, fractional reduction of $\mathrm{Fe}^{3+}$ atoms in $\mathrm{Fe}_{2}(\mathrm{BDP})_{3}(\mathrm{BDP}=1$,4benzenedipyrazolate) to access mixed valence state of $\mathrm{Fe}^{2 / 3+}$ in the framework resulted in a nearly four orders of magnitude higher conductivity along a single crystallographic axis. ${ }^{115}$ For 2D $c$-MOF systems, many examples possess mixed valence as well, such as $\mathrm{Fe}^{2 / 3+}, \mathrm{Co}^{2 / 3+}$, and $\mathrm{Cu}^{1 / 2+} \cdot 33,39,56,80,118$ Nevertheless, the valence influence on the electronic properties has not been systematically investigated due to the lack of control on the metal valence. For the moment, we would assume a similar conclusion in 2D $c$-MOFs compared with conductive 3D MOFs.

On the other hand, the metal type also plays an important role in the charge transport performance of $2 \mathrm{D} c$-MOFs. ${ }^{58,119}$ For instance, first-principles calculations indicated that the band gaps of $\mathrm{M}_{3}(\mathrm{HTB})(\mathrm{M}=\mathrm{Mg}, \mathrm{Ca}, \mathrm{Zn}, \mathrm{Cd}, \mathrm{Ge}$, and $\mathrm{Sn})$ ranged from 1.7 to $3.2 \mathrm{eV}^{120}$ Besides, the $\pi-\mathrm{d}$ hybridization between $\mathrm{d}$ (metal) and $\pi$ (HTB) bands can considerably delocalize the wave function of band edge states and is of importance in determining their hole effective masses. More specifically, as the $\pi-\mathrm{d}$ coupling increases from the elements of group IIA $(\mathrm{Mg}, \mathrm{Ca})$ to 


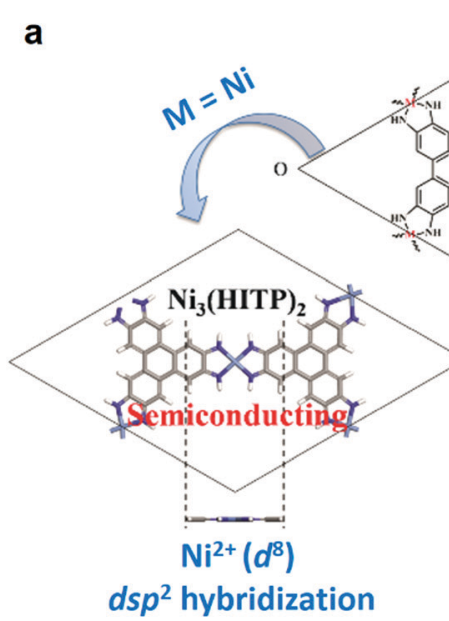

C

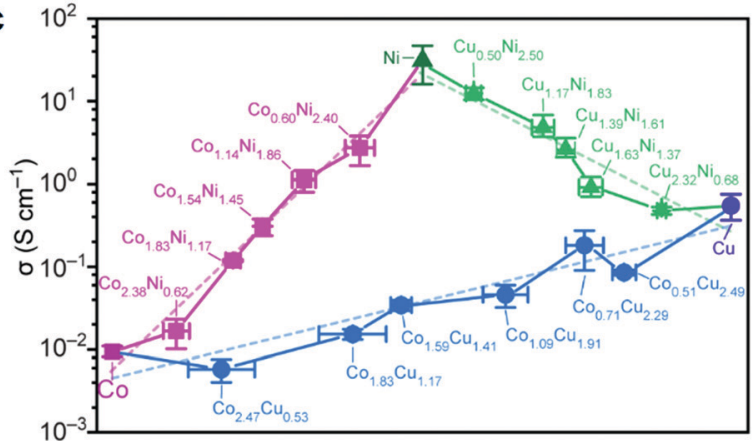

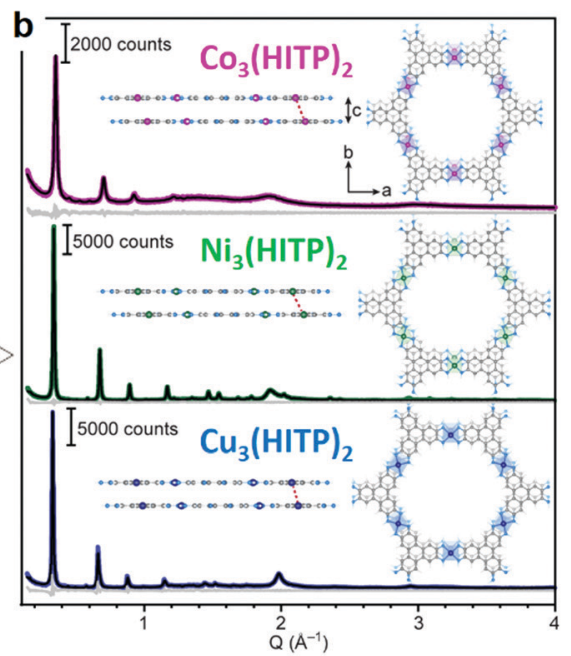

d

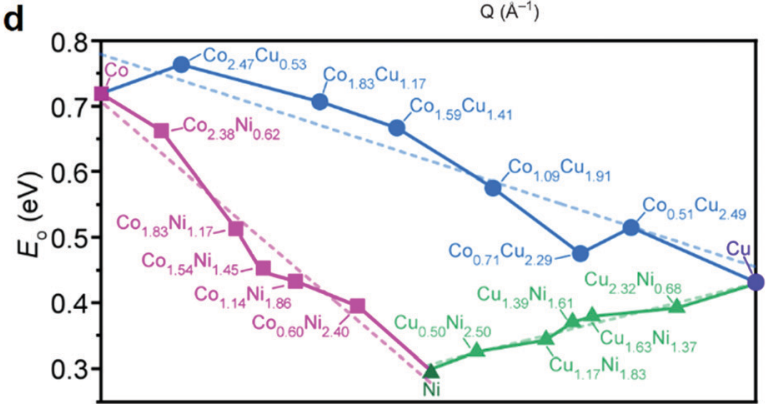

Fig. 6 Metal substitution in 2D c-MOFs. (a) Illustration of the geometry of $M_{3}(\mathrm{HITP})_{2}\left(\mathrm{M}=\mathrm{Ni}\right.$, Cu) by substituting Ni (left) with Cu (right). ${ }^{113}$ Copyright 2015, the PCCP Owner Societies. (b) Synchrotron X-ray diffraction patterns and Pawley refinements for $M_{3}(H I T P)_{2}$. The insets show the bilayer of $M_{3}(\mathrm{HITP})_{2}$ with reference to the interlayer displacement. (c and d) Electrical conductivity and optical bandgap of $M_{n} M_{3-n}^{\prime}(\mathrm{HITP})_{2}$, respectively. Dashed lines refer to linear fitting. Reproduced with permission ref. 58. Copyright 2020, American Chemical Society.

IVA (Ge, Sn) to IIB (Zn, Cd), the hole effective masses decrease significantly. While for electron transport, from group IIB (Zn, Cd) to IIA ( $\mathrm{Mg}, \mathrm{Ca})$ to IVA (Ge, Sn), the electron effective masses increase. ${ }^{120}$ Nonetheless, these 2D $c$-MOFs have so-far not been constructed in the laboratory. In addition, great efforts from DFT calculations have been also devoted to the well-known $\mathrm{M}_{3}(\mathrm{HITP})_{2}(\mathrm{M}=\mathrm{Ni}, \mathrm{Cu})$ 2D $c$-MOFs regarding the metal substitution. The substitution from $\mathrm{Ni}$ to $\mathrm{Cu}$ in this system will change both the geometries and the electronic band structures (Fig. 6a). The Ni atoms in $\mathrm{Ni}_{3}$ (HITP) $)_{2}$ adopt the dsp ${ }^{2}$ hybridization in a square-planar geometry, thus forming a planar $2 \mathrm{D}$ framework. ${ }^{113}$ Nevertheless, for $\mathrm{Cu}_{3}(\mathrm{HITP})_{2}$, the $\mathrm{Cu}$ atoms adopt the $\mathrm{sp}^{3}$ hybridization leading to a distorted network in a specific square-grid coordination geometry. In addition, a single-layer of $\mathrm{Cu}_{3}(\mathrm{HITP})_{2}$ is metallic whilst the monolayer of $\mathrm{Ni}_{3}(\text { HITP })_{2}$ is an indirect semiconductor. ${ }^{113}$

Experimentally, varying the metal center in the chargeneutral $\mathrm{M}_{3}(\mathrm{HITP})_{2} 2 \mathrm{D} c$-MOF system could readily change the layer distance, which was determined as 3.30, 3.16, and $3.29 \AA$ for $\mathrm{Ni}_{3}(\mathrm{HITP})_{2}, \mathrm{Cu}_{3}(\mathrm{HITP})_{2}$ and $\mathrm{Co}_{3}(\mathrm{HITP})_{2}$, respectively (Fig. 6b). ${ }^{58}$ The layer distance refers to the strength of $\pi-\pi$ stacking interactions, thereby enabling to define the electronic structure in the cross-plane direction (Section 3.5). According the PXRD analysis, the substitution of the metal center is also relevant to the interlayer displacement, which was recorded as 1.56, 0.86, and $1.39 \AA$ respectively for the above 2D $c$-MOFs. Unsurprisingly, conductivities of these 2D $c$-MOF samples differed greatly, which were determined as 55.4, 0.75, and $0.024 \mathrm{~S} \mathrm{~cm}^{-1}$, respectively. In this work, Dincă and coworkers further demonstrated a series of binary alloys of $\mathbf{M}_{n} \mathbf{M}_{3-n}^{\prime}(\mathrm{HITP})_{2}$ consisting of two metal species $(\mathrm{Co} / \mathrm{Cu}, \mathrm{Co} /$ $\mathrm{Ni}$, or $\mathrm{Cu} / \mathrm{Ni}$ ) within one network. ${ }^{58}$ Continuous shifts of the interlayer distance and displacement as well as conductivity were determined with an increasing amount of one metal. For instance, increasing the amount of $\mathrm{Ni}$ in the $\mathrm{Co}_{n} \mathrm{Ni}_{3-n}(\mathrm{HITP})_{2}$ system shifts the values of layer distance and displacement in such a way as to enhance the $\pi-\pi$ interactions, which results in a continuous increment of conductivity over 3 orders of magnitude from 0.024 to $55.4 \mathrm{~S} \mathrm{~cm}^{-1}$ (Fig. 6c) and a continuously tuned bandgap over $0.4 \mathrm{eV}$ in fine-scale (Fig. 6d). While for the fully substituted coronene (PTC), the metal substitution from Fe to Ni/ $\mathrm{Cu}$ results in not only changes in the conductivity but also in the variation of the chemical structures of 2D $c$-MOFs (Fig. 2c and d). ${ }^{32,110,111}$ In addition, the metal atoms play also an important role in the magnetic properties of $2 \mathrm{D} c$-MOFs. In particular, due to the unique spin properties of Fe and the strong $\pi-\mathrm{d}$ hybridization between the $\mathrm{d} / \mathrm{p}$ orbitals in the $\mathrm{FeX}_{4}$ complex, the resultant $2 \mathrm{D}$ $c$-MOFs exhibit nontrivial ferromagnetism (Section 6.3). ${ }^{32,33}$ 


\subsection{Effect of redox-activity in linkage}

Here, the redox activity majorly refers to the charge state of the linkages. Each linkage is able to undergo a two-electron transfer through redox reactions (Fig. 7), which corresponds to diverse charge state of the linkage as 0 (existence of monoradical), -1 (presence of counter cation), and 1 (presence of counter anion). Among the reported 2D $c$-MOFs, the ligands are generally partially oxidized providing free radicals as charge carriers (charge-neutral framework with a charge state of 0 ), which correlates with the density $(n)$ of charge carriers in the frameworks. ${ }^{19,36,51,56,65,73-75}$ For example, $\mathrm{Cu}_{3}(\mathrm{HHB})_{2}$ possesses a network with denser distributed ligands and metals as well as smaller layer distance $(\sim 3 \AA)^{39}$ compared with $\mathrm{Cu}_{3}(\mathrm{HHTP})_{2}$ $(\sim 3.3 \AA) ;{ }^{95}$ the former one was expected to display higher conductivity due to the stronger cross-plane $\pi-\pi$ interactions (Section 3.5). However, conductivity experiments indicated inferior values in the range of $(0.22-15) \times 10^{-8} \mathrm{~S} \mathrm{~cm}^{-1}$ for $\mathrm{Cu}_{3}(\mathrm{HHB})_{2}$, making it like an insulator. The huge difference would be attributed to the localized charge carriers due to the absence of free radicals with respect to a charge state of -1 . While for $\mathrm{Ni}_{3}(\mathrm{HTB})_{2}$, X-ray photoelectron spectroscopy (XPS) analysis revealed mixed charge states of 0 and -1 (ratio of $26: 74$, corresponding to an average charge state of $-3 / 4$ ) in the nickel-bis(dithiolene) linkages for the as-synthesized sample (conductivity in the range of $0.15-2.8 \mathrm{~S} \mathrm{~cm}^{-1}$ ), indicative of the existence of few free radicals. It was demonstrated that additionally generated radicals upon oxidation offer abundant free carriers, thereby leading to enhanced conductivity values. ${ }^{74,75}$ For instance, the amount of radicals in $\mathrm{Ni}_{3}(\mathrm{HTB})_{2}$ was further increased through oxidation reactions (charge state of 0 ), leading to significantly advanced conductivity up to $160 \mathrm{~S} \mathrm{~cm}^{-1}$. By comparison, the reduction reaction on the as-synthesized $\mathrm{Ni}_{3}(\mathrm{HTB})_{2}$ led to a decreased conductivity of $6.7 \times 10^{-3} \mathrm{~S} \mathrm{~cm}^{-1}$, which can be ascribed to the disappearance of those few radicals after chemical reduction (charge state of -1 ).

\subsection{Effect of layer stacking and arrangement}

As is seen in 2D c-MOFs, linking the ortho-substituted conjugated ligands by square-planar metal coordination complexes yields an extended $\pi$-conjugated network, thus enhancing the delocalization of charge carriers within the 2D plane. The in-plane charge carrier migration was considered as the dominant pathway of the intrinsic charge transport in 2D $c$-MOFs. Nevertheless, in recent studies, DFT calculations and charge transport characterization based on single-crystals revealed the significance of the through-space pathway in the electronic properties. As mentioned in Section 3.3, a single-layer of $\mathrm{Ni}_{3}(\mathrm{HITP})_{2}$ is predicted to be a semiconductor (bandgap of $0.13 \mathrm{eV}$ ) (Fig. 8a and b). The increased dimensionality from a

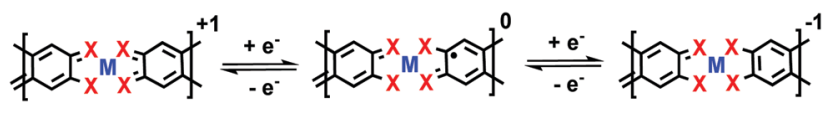

Fig. 7 Redox activity of the linkage of 2D c-MOFs through electron transfer. monolayer to layer-stacked $\mathrm{Ni}_{3}(\mathrm{HITP})_{2}$ leads to a significant change from semiconducting to metallic in the electronic band structure (Fig. 8c), which can be attributed to the strong $\pi-\pi$ $(d=3.3 \AA)$ and weak Ni-Ni $(d=3.809 \AA)$ interactions in the direction perpendicular to the $\mathrm{Ni}_{3}(\mathrm{HITP})_{2}$ layers. ${ }^{113}$ Very recently, the Dincă group characterized the out-of-plane conductivity of $\mathrm{Ni}_{3}(\mathrm{HITP})_{2}$ up to $150 \mathrm{~S} \mathrm{~cm}^{-1}$ and demonstrated that interlayer $\pi-\pi$ interactions indeed considerably contribute to the conductivity in layer-stacked 2D c-MOFs based on singlecrystalline samples and related devices. ${ }^{95}$ Thus, it raised up an open question whether high-performance electrically conductive MOFs necessarily require the incorporation of in-plane conjugated structures. Another work from the same group reported layered lanthanide-HHTP MOFs with metal centers located in between the linker layers. ${ }^{121}$ The lanthanide-HHTP MOFs do not possess in-plane $\pi$-d conjugation but nevertheless display high electrical conductivity (up to $0.05 \mathrm{~S} \mathrm{~cm}^{-1}$ ).

Rational tuning the interlayer spacing and modification of layer arrangement can induce significant changes in the electronic band structures as well as conductivities. Great efforts from DFT calculations have been devoted to the calculation of band structures and electrical properties for $2 \mathrm{D}$ $c$-MOFs. ${ }^{31-33,40,78-80,112,113,122-127}$ Combining experimental and computational analysis, the reported 2D $c$-MOFs preferred to stack in an AA-eclipsed (AAAA) symmetry (Fig. 8d), rather than the staggered $\mathrm{AB}(\mathrm{ABAB})$ mode. ${ }^{46,48,50,51,54}$ Taking carefully in the $\mathrm{AA}$ symmetry, the AA-serrated $\left(\mathrm{AA}^{\prime} \mathrm{AA}^{\prime}\right)$ stacking (Fig. 8e), so called "slipped-parallel $\mathrm{AB}$ stacking" with slightly slipped arrangement in every two layers, ${ }^{19,28,32,33,36,39,40,42,52,53,57,59,68,96,97,99}$ and AAinclined $\left(\mathrm{AA}^{\prime} \mathrm{A}^{\prime \prime} \mathrm{A}^{\prime \prime \prime}\right.$, that is, the layers are continuously shifted in one direction) stacking (Fig. 8f) ${ }^{78}$ were suggested to be energetically more favored than the AA-eclipsed one in these highly conjugated systems. The stacking-refers to the arrangement of layers-affects the pore geometries ${ }^{128}$ and the electronic properties $^{129}$ of $2 \mathrm{D}$ framework materials. Taking a representative 2D covalent organic framework (COF), COF- $5,{ }^{130}$ as an example, the band structure of a single-layer shows flat conduction and valence bands as well as typical signature of the kagome lattice $^{68,112,113}$ along the $\Gamma-K-M-\Gamma$ path (in-plane), while stacking the layers with different arrangements varies not only the out-ofplane charge transport, but also the in-plane one (Fig. $8 \mathrm{~d}-\mathrm{g}$, bottom). ${ }^{129}$ The AA-eclipsed stacked COF-5 retains almost unchanged band structures with preserved signature of the kagome lattice in the in-plane direction (for details see ref. 129), while interlayer interactions induce strong cross-plane band dispersion hence narrowing the band gap from $\sim 2.5$ (monolayer) to $1.3 \mathrm{eV}$. However, the AA-serrated and AA-inclined stacking modes strongly alter the in-plane electronic structure, leading to the absence of the kagome characteristic (for details see ref. 121). Compared with the eclipsed layer arrangement, both the serrated and inclined cases exhibit smaller out-of-plane dispersion due to the weaker $\pi-\pi$ interactions, which corresponds to a larger band gap of $2.2 \mathrm{eV}$ for both cases. In another instance, $\mathrm{Fe}_{3}(\mathrm{HTTP})_{2} 2 \mathrm{D}$ $c$-MOF, the band gap of a monolayer was calculated as $0.3 \mathrm{eV}$; it opens up to 0.35 and $0.65 \mathrm{eV}$ for the AA-inclined and AA-eclipsed arrangements, respectively. ${ }^{78}$ Furthermore, the obtained effective 

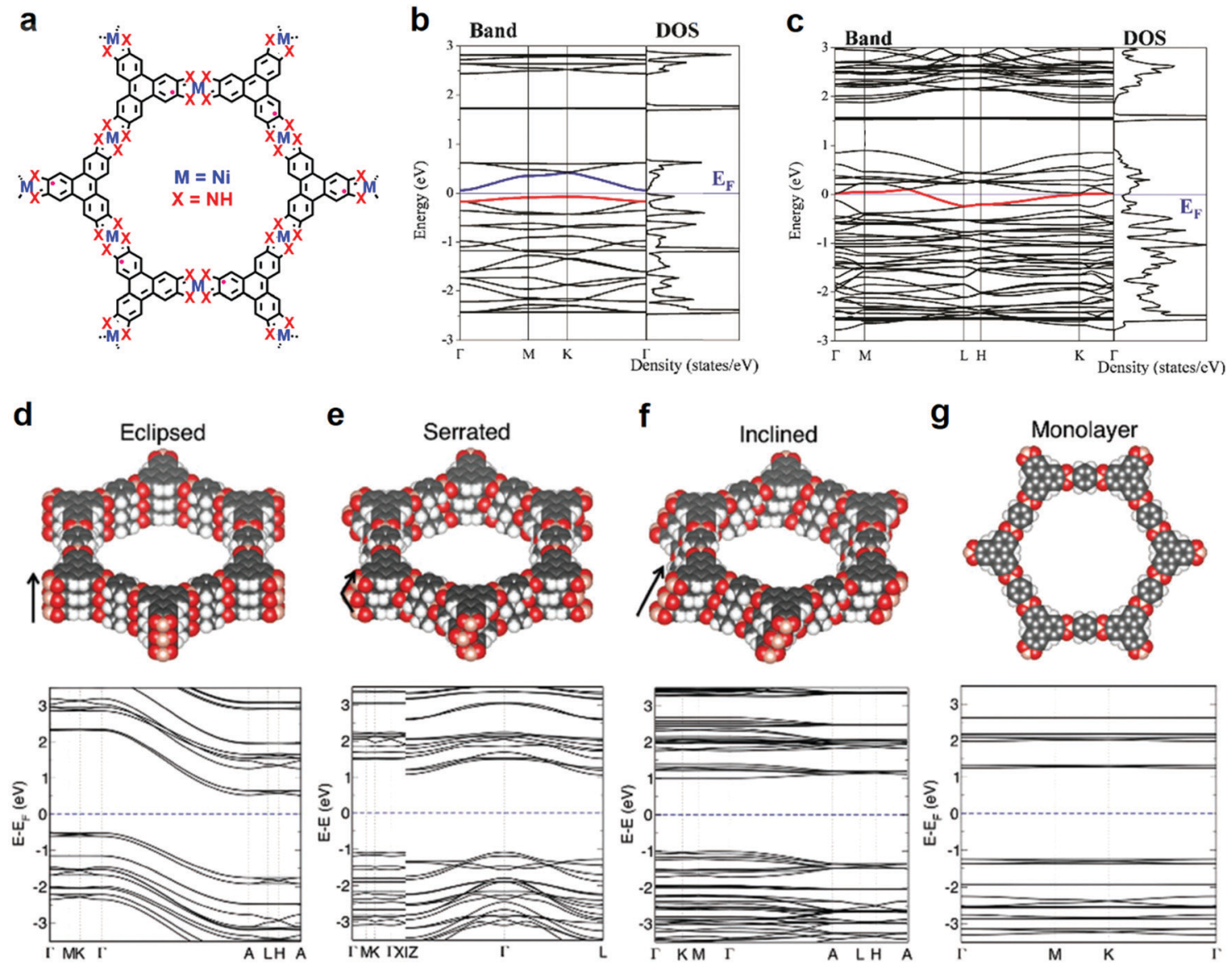

Fig. 8 Electronic band structure with respect to layer stacking and arrangement. (a-c) Band structure for monolayer (b) and layer-stacked (c) $\mathrm{Ni}_{3}(\mathrm{HITP})_{2} .{ }^{113}$ Copyright 2015, the PCCP Owner Societies. (d-f) AA-eclipsed, AA-serrated, and AA-inclined stacking of COF-5 as well as related band structures shown at the bottom. (g) Monolayer and related band structures of COF-5. ${ }^{129}$ Copyright 2020, Wiley-VCH.

mass $\left(m^{*}\right)$ values for AA-inclined stacking $\left(m_{h}^{*}=0.78, m_{e}^{*}=0.98\right)$ and AA-eclipsed stacking $\left(m_{h}^{*}=1.07, m_{e}^{*}=2.08\right)$ differ significantly from those for a monolayer $\left(m_{h}^{*}=0.45, m_{e}^{*}=1.34\right)$ of $\mathrm{Fe}_{3}(\mathrm{HTTP})_{2}$. Therefore, to evaluate the band diagrams in the absence of high structural resolution, several potential stacking sequences including not merely $\mathrm{AA}$-eclipsed and $\mathrm{AB}$ arrangements merit thorough consideration.

In addition to the foregoing points, high crystallinity or even single-crystal not only offers a high-quality sample but also allows precise structural analysis, ${ }^{71,131-133}$ thus ensuring the establishment of a reliable structure-property relationship. The polycrystalline $\mathrm{Cu}_{3}(\mathrm{HITP})_{2}$ possessed a mixed valence of $\mathrm{Cu}^{1 / 2+}$ in the as-synthesized sample. ${ }^{28}$ However, all $\mathrm{Cu}$ atoms presented to be $\mathrm{Cu}^{2+}$ in the single-crystalline sample hence ruling out the contribution of mixed valences to conductivity. ${ }^{58}$ The mixed valence of $\mathrm{Cu}^{1 / 2+}$ could be ascribed to the presence of defects or amorphous regions in the less crystalline $\mathrm{Cu}_{3}(\mathrm{HITP})_{2}$ sample. Furthermore, the conductivity value of the compressed pellet of $\mathrm{Cu}_{3}(\mathrm{HITP})_{2}$ single-crystals increased from $0.2 \mathrm{~S} \mathrm{~cm}^{-1}$ for the polycrystalline $\mathrm{Cu}_{3}(\mathrm{HITP})_{2}$ to $0.75 \mathrm{~S} \mathrm{~cm}^{-1}$. In another example, the highly crystalline $\mathrm{Cu}_{3}(\mathrm{HTB})$ film exhibits a conductivity reaching $2500 \mathrm{~S} \mathrm{~cm}^{-1},{ }^{68}$ while the conductivity of the one with low crystallinity (according to the X-ray diffraction and transmission electron microscopy analysis) was merely determined to be $1580 \mathrm{~S} \mathrm{~cm}^{-1} \cdot{ }^{31,134}$ In addition, the synthesized $\mathrm{M}_{3}(\mathrm{HIB})_{2}(\mathrm{M}=\mathrm{Cu}, \mathrm{Ni}, \mathrm{Co})$ films were reported as an electrical insulator in the early work from Lahiri et al. ${ }^{135}$ which is likely attributed to the low crystallinity in these samples. By comparison, their intrinsic metallicity was confirmed in the highly crystalline bulk samples $(\mathrm{M}=\mathrm{Cu}, \mathrm{Ni})$ by ultravioletphotoelectron spectroscopy. Conductivity measurements on the pellets revealed characteristic values of 8 and $13 \mathrm{~S} \mathrm{~cm}^{-1}$ for $\mathrm{Ni}_{3}(\mathrm{HIB})_{2}$ and $\mathrm{Cu}_{3}(\mathrm{HIB})_{2}$, respectively. ${ }^{40}$ The polycrystalline $\mathrm{Ni}_{3}(\mathrm{HITP})_{2} 2 \mathrm{D} c$-MOF displayed temperature-dependence suggesting a semiconducting behavior and a conductivity up to $\sim 60 \mathrm{~S} \mathrm{~cm}{ }^{-1},{ }^{57}$ while single-crystalline $\mathrm{Ni}_{3}(\mathrm{HITP})_{2}$ nanorod sample presented metallicity with a higher conductivity of $\sim 150 \mathrm{~S} \mathrm{~cm}^{-1}$ through the cross-plane direction. ${ }^{95}$ Thus, single-crystalline devices that can minimize the influence of grain boundary and disorder on the charge transport are urgently required for characterizing 2D $c$-MOFs. ${ }^{29,57}$ Besides conductivity, few reports attempted to probe the type of carriers and charge mobility as well as density for $2 \mathrm{D} c$-MOFs in the direct current (DC) limits including field-effect transistors and Hall effect measurements, as well as in the alternating current (AC) limits such as contact-free time-resolved terahertz 
spectroscopy (TRTS) and flash photolysis-time-resolved microwave conductivity. ${ }^{33}$ An in-depth understanding of the mechanism underlying the conductivity and the establishment of a reliable structure-property relationship through combining multi-scaled physical characterization methods merit further exploration.

\section{Synthetic methodologies of 2D c-MOFs}

Currently, various synthetic methodologies have been applied to develop 2D $c$-MOFs as bulk or film samples. Hydro-/solvothermal syntheses represent a representative strategy (Fig. 9) that allows diversity in the reaction conditions including the solvent, temperature, volume and pressure, and offers advantages in scalable and high-yield synthesis. They also provide the possibility to achieve moderate-sized $(1-10 \mu \mathrm{m})$ single-crystals. ${ }^{19,95}$ Nevertheless, the obtained bulk materials generally comprise nanocrystals smaller than 100 nanometers (nm), associating with difficulties in sample processing, device fabrication, and charge transport characterization. Recently, the wet-interface-assisted synthesis ${ }^{21,136-142}$ shows promise in preparing crystalline, large-area, free-standing 2D $c$-MOF thin films. These films ensure facile device integration (notably for electronic devices) and sufficient charge transport ${ }^{29,31,36,41,57,68,74,75,78-80,96,97,143}$ that holds potential to realize the practical applications of these materials for MOFtronics. Nonetheless, the universality of this interfacial strategy towards large $\pi$-conjugated systems, the further improvement of the crystallinity and the mechanical strength of the film as well as the control of the film thickness are still synthetic challenges. In the following sections, we will introduce these

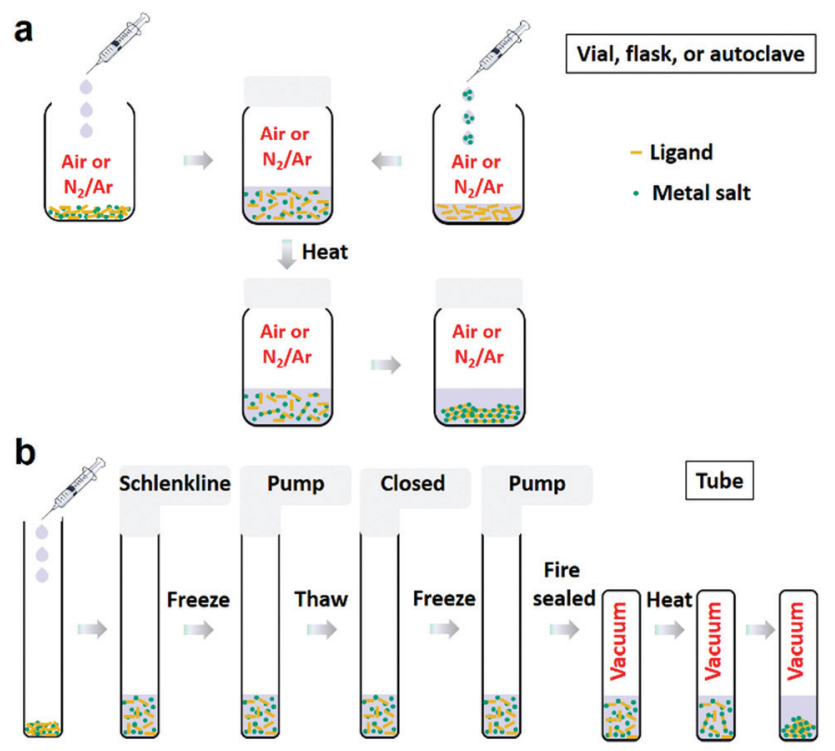

Fig. 9 Schematic illustration of various hydro-/solvothermal syntheses methods. (a) Under ambient or inert atmosphere. (b) Under vacuum. synthetic methodologies toward 2D $c$-MOFs and discuss the current achievements and challenges.

\subsection{Bulk 2D c-MOFs through hydro-/solvothermal syntheses}

In 2012, Hmadeh et al. reported a series of TP-based $\mathrm{MO}_{4}$ linked 2D $c$-MOFs by heating an aqueous solution of HHTP and metal(II) acetate $(\mathrm{M}=\mathrm{Cu}, \mathrm{Ni}, \mathrm{Co})$ at $85{ }^{\circ} \mathrm{C}$ for 24 hours (Fig. 9a and 10a). ${ }^{19}$ Scanning electron microscopy (SEM) revealed uniform morphology of the obtained samples as nanorods with length less than $10 \mu \mathrm{m}$ and diameter of $\sim 100 \mathrm{~nm}$ (Fig. 10e). Their crystal structures were resolved by single-crystal X-ray diffraction, PXRD, and high-resolution transmission electron microscopy (HR-TEM). These 2D $c$-MOFs possess two different structures: (1) $\mathrm{Cu}_{3}(\mathrm{HHTP})_{2}$ (Fig. 10b) with a layered honeycomb network and (2) $\mathbf{M}_{9}(\text { HHTP })_{4}$ (discounting water molecules, $\mathbf{M}=$ Co, Ni) (Fig. 10c) comprising alternatively layered honeycomb frameworks of $\mathrm{M}_{3}(\mathrm{HHTP})_{2}\left(\mathrm{H}_{2} \mathrm{O}\right)_{6}$ (each $\mathrm{M}^{2+}$ is coordinated to the linkers in two adjacent deprotonated HHTP molecules and two additional aqua ligands in an octahedral environment) and trinuclear $\mathrm{M}_{3}(\mathrm{HHTP})\left(\mathrm{H}_{2} \mathrm{O}\right)_{12}$ clusters (one $\mathrm{M}^{2+}$ is coordinated to the linkers in one HHTP molecules and four additional aqua ligands) (Fig. 10d). ${ }^{99}$ Notably, the $\mathrm{Cu}^{2+}$ ions in $\mathrm{Cu}_{3}(\mathrm{HHTP})_{2}$ are in a square-planar environment without the presence of water ligands. The Brunauer-Emmett-Teller (BET) measurements
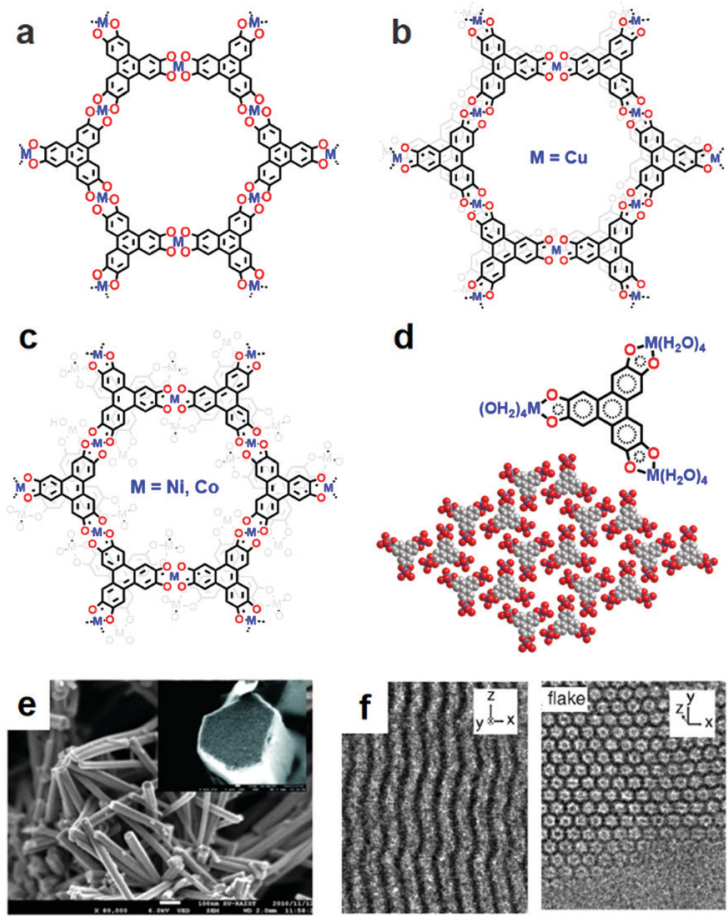

Fig. 10 TP-based 2D c-MOFs through hydro-/solvothermal syntheses. (a) Generally displayed structure of $\mathrm{Cu}_{3}(\mathrm{HHTP})_{2}$ and $\mathrm{M}_{9}(\mathrm{HHTP})_{4}(\mathrm{M}=\mathrm{Ni}, \mathrm{Co})$. (b) Radical structure of bilayer $\mathrm{Cu}_{3}(\mathrm{HHTP})_{2}$. (c) Radical structure of layered $\mathrm{M}_{9}(\mathrm{HHTP})_{4}(\mathrm{M}=\mathrm{Ni}, \mathrm{Co})$ without counting the water molecules. (d) Structure of trinuclear $\mathrm{M}_{3}(\mathrm{HHTP})\left(\mathrm{H}_{2} \mathrm{O}\right)_{12}$ clusters. (e) SEM image of the nanorod sample. Reproduced with permission ref. 19. Copyright 2012, American Chemical Society. (f) HR-TEM images of $\mathrm{Cu}_{3}(\mathrm{HHTP})_{2}$ nanorods (left) and nanoflakes (right). Reproduced with permission ref. 95. Copyright 2019, American Chemical Society. 
displayed the specific surface area (SSA) values of these porous materials in the range of 400 to $500 \mathrm{~m}^{2} \mathrm{~g}^{-1}$. Electron paramagnetic resonance (EPR) suggested a ligand-centered monoradical (Fig. 7, charge state of 0) and confirmed the charge-neutral nature (Fig. 10b and c). In a later report from Day et al., $\mathrm{Cu}_{3}(\mathrm{HHTP})_{2}$ layers stacked perpendicular to the axis along each nanorod in a slightly offset fashion with a continuous shift in the c direction (Fig. 10f, left). ${ }^{95}$ Combining liquid-phase exfoliation, ${ }^{144,145}$ the authors further synthesized single-crystalline nanoflake-like $\mathrm{Cu}_{3}(\mathrm{HHTP})_{2}$ (Fig. 10f, right).

In addition to $\mathrm{Cu}_{3}(\mathrm{HHTP})_{2}, \quad \mathrm{NiN}_{4}$-linked $\mathrm{Ni}_{3}(\mathrm{HITP})_{2}$ is another representative 2D c-MOF sample. In 2014, Sheberla et al. reported the hydrothermal synthesis of $\mathrm{Ni}_{3}(\mathrm{HITP})_{2}$ based on amine-functionalized TP. ${ }^{57}$ Upon heating an aqueous solution of HATP and $\mathrm{NiCl}_{2}$ with the existence of $\mathrm{NH}_{3} \cdot \mathrm{H}_{2} \mathrm{O}$ in air, $\mathrm{Ni}_{3}(\mathrm{HITP})_{2}$ was obtained as bulk nanoparticles with an average crystal size less than $200 \mathrm{~nm}$. Monoanionic $o$-diiminobenzosemiquinonate moieties were confirmed by XPS and elemental analysis, indicative of a charge-neutral network. The further structural analysis revealed the crystal structure of $\mathrm{Ni}_{3}(\mathrm{HITP})_{2}$ with unit cell parameters of $a=b=21.75 \AA$. In the later studies from the same group, employing NaOAc for the deprotonation, the obtained $\mathrm{Ni}_{3}(\mathrm{HITP})_{2}$ showed superior crystallinity in a rod-like morphology with length of up to $2 \mu \mathrm{m} .{ }^{58,95}$ The HR-TEM image of the individual crystal suggested that the $\mathrm{Ni}_{3}(\mathrm{HITP})_{2}$ layers stacked in an almost eclipsed configuration with a periodic interlayer spacing of $3.30 \AA$. This optimization was also applied to the structurally related charge-neutral $\mathrm{Cu}_{3}(\mathrm{HITP})_{2}$ and $\mathrm{Co}_{3}(\mathrm{HITP})_{2} \quad 2 \mathrm{D} \quad c$-MOFs. ${ }^{28,58}$ Meanwhile, HTTP-based $\mathrm{MS}_{4}$-linked ( $\left.\mathrm{M}=\mathrm{Ni}, \mathrm{Co}, \mathrm{Fe}, \mathrm{Pt}\right)$ 2D $c$-MOFs were also developed (mainly as films, see Section 4.2), ${ }^{45,64,118}$ and subsequently great efforts have been devoted to the TP-based 2D $c$-MOF family with the aim of exploring their multifunctional properties. ${ }^{28,46,59,90,100,106,109,146,147}$

Towards frameworks with denser distributed ligands and metals, benzene-based ligands were developed. ${ }^{31,39,40,42,74,135}$ Typically, in 2017, Dou et al. synthesized $\mathrm{MN}_{4}$-linked $\mathrm{M}_{3}(\mathrm{HIB})_{2}$ $(\mathrm{M}=\mathrm{Cu}, \mathrm{Ni}) 2 \mathrm{D} c$-MOFs by mixing $\mathrm{HAB}, \mathrm{M}^{2+}$, and $\mathrm{NH}_{3} \cdot \mathrm{H}_{2} \mathrm{O}$ in DMSO, followed by heating at $60{ }^{\circ} \mathrm{C}$ for 2 hours. ${ }^{40}$ The obtained samples were formed by irregularly shaped nanoparticles smaller than $100 \mathrm{~nm}$. The backbones were suggested to be charge-neutral, indicative of the existence of same monoanionic $o$-diiminobenzosemiquinonate moieties as $\mathrm{Ni}_{3}(\mathrm{HITP})_{2}$. Unsurprisingly, $\mathrm{N}_{2}$ adsorption revealed low BET SSA values in the range of $110-150 \mathrm{~m}^{2} \mathrm{~g}^{-1}$ due to the increment of the density of ligands and metals in the backbone. Using ethylenediamine as the base under an inert atmosphere, Park et al. reported $\mathrm{CuO}_{4}$-linked $\mathrm{Cu}_{3}(\mathrm{HHB})_{2} 2 \mathrm{D} c$-MOF nanocrystals (estimated to be $<200 \mathrm{~nm}){ }^{39}$ The authors showed synthetic diversity through the coordination reaction between $\mathrm{Cu}^{2+}$ and either HHB or its derivative tetrahydroxyquinone (THQ). Elemental analysis suggested the presence of protonated ethylenediamine $\left(\mathrm{NH}_{3} \mathrm{CH}_{2} \mathrm{CH}_{2} \mathrm{NH}_{3}{ }^{2+}\right)$ as the counter cation to balance the negative charge of the backbones, which corresponds to a charge state of -1 in each linkage. Analogous to $\mathrm{M}_{3}(\mathrm{HIB})_{2}$, $\mathrm{Cu}_{3}(\mathrm{HHB})_{2}$ exhibited a low BET SSA value of $\sim 143 \mathrm{~m}^{2} \mathrm{~g}^{-1}$.
A larger $\pi$-conjugated coronene-based building block was introduced for the construction of 2D $c$-MOFs by our group in 2016. ${ }^{32}$ The fully thiol-substituted ligand PTC was incorporated into the 2D $c$-MOF through the coordination with $\mathrm{Fe}(\mathrm{OAc})_{2}$ in an ammoniacal mixture containing deoxygenated water and dimethylformamide (DMF) at $120{ }^{\circ} \mathrm{C}$ for 48 hours. Thus, $\mathrm{FeS}_{4}$ linked $\mathrm{Fe}_{3}(\mathrm{PTC})_{2}$ was achieved as an almost non-porous network with crystalline domains up to a dozen of nm. XPS and Mössbauer spectroscopy suggested the presence of $\mathrm{Fe}^{3+}$ species. In addition, we inferred a unit cell with $a=b=\sim 11.7 \AA$ and an interlayer spacing of $\sim 3.9 \AA$ for this $2 \mathrm{D} c$-MOF depending on thorough characterization with the support of PXRD, TEM, XPS, and X-ray absorption spectroscopy. Very recently, Yao et al. established a dual-ligand $\mathrm{Cu}_{3}(\mathrm{HHTP})(\mathrm{HHB}) 2 \mathrm{D} c$-MOF with two kinds of ligands of HHTP and THQ in the network. ${ }^{59}$ Definitely, it was a challenge for the controlled synthesis of this dualligand 2D $c$-MOF by varying various reaction conditions including the temperature, reaction time, ratio of two ligands, and metal salts, in terms of stronger coordination capability of $\mathrm{Cu}^{2+}$ with HHTP than THQ. As a result, the obtained $\mathrm{Cu}_{3}$ (HHTP)(HHB) 2D $c$-MOF presented a morphology of hundreds of nm long nanowires and a unit cell with parameters of $a=b=17.154 \AA$ and $c=6.266 \AA$ for a honeycomb structure. In addition, the charge state of the linkage was -1 with $\mathrm{NH}_{3} \mathrm{CH}_{2} \mathrm{CH}_{2} \mathrm{NH}_{3}{ }^{2+}$ as counter ions. The dual-ligand structure shows the great potential of tunability of the porosity and electronic properties.

Utilizing hydro-/solvothermal methodologies, many other 2D $c$-MOFs based on various building blocks have been constructed, such as $\mathrm{Cu}_{3}(\mathrm{HHTN})_{2}, \mathrm{Cu}_{3}(\mathrm{HHTX})_{2}, \mathrm{Ni}_{3}\left(\mathrm{HIHATNM}_{3}\right)_{2}$, $\mathrm{Cu}_{2}\left[\mathrm{DBCO}_{8}\right], \mathrm{M}_{2}\left[\mathrm{M}^{\prime} \mathrm{PcX}_{8}\right]$, and $\mathrm{Ni}_{2}\left[\mathrm{NiNPcO}_{8}\right] .^{33,47-51,53-56,148}$ In 2019, we employed a modified solvothermal protocol applying vacuum for the synthesis of an oxygen-sensitive system (Fig. 9b): $\mathrm{Fe}_{2}\left[\mathrm{FePcO}_{8}\right]$ 2D $c$-MOF. ${ }^{33}$ This method has also been widely employed for vacuum-promoted synthesis of $2 \mathrm{D}$ $\mathrm{COFs}^{148-150}$ and 2D non-conjugated MOFs. ${ }^{151}$ To avoid airinduced oxidation of iron(II) salts, $\mathrm{Fe}_{2}\left[\mathrm{FePcO}_{8}\right]$ was synthesized by mixing $\mathrm{FePc}(\mathrm{OH})_{8}, \mathrm{Fe}(\mathrm{OAc})_{2}$, and KOAc (base) in $N$-methyl-2pyrrolidone $(\mathrm{NMP}) / \mathrm{H}_{2} \mathrm{O}(\mathrm{v} / \mathrm{v}=3 / 1)$ in a vacuum-sealed tube after thorough deoxygenation by three cycles of freeze-pump-thaw, followed by heating at $150{ }^{\circ} \mathrm{C}$ for 3 days. Thus, the obtained $\mathrm{Fe}_{2}\left[\mathrm{FePcO}_{8}\right]$ is comprised of rectangle-shaped crystals in the range of 10-100 nm with a square lattice of $a=b=\sim 18 \AA$.

Though hydro-/solvothermal syntheses have been demonstrated as the most effective and simplest strategy for expanding the 2D $c$-MOF family, this method generally allows barely monitoring or controlling the crystal growth and provides small-sized bulk polycrystals. As a result, integrating the MOF particles into nanodevices and the relevant performance of the thus-far fabricated devices have been largely impeded by the lack of process-capability and accessible active sites as well as sufficient charge carrier transport.

\subsection{D $c$-MOF films through wet-interface-assisted synthesis}

Recently, various bottom-up wet interface-assisted synthetic strategies including gas-liquid (GL), liquid-liquid (LL), and 


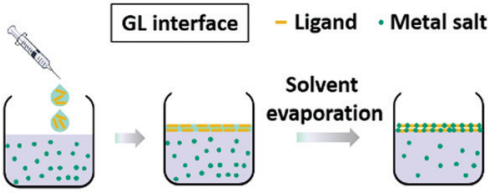

b

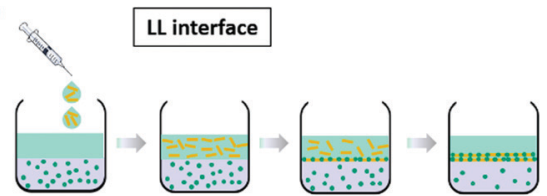

c

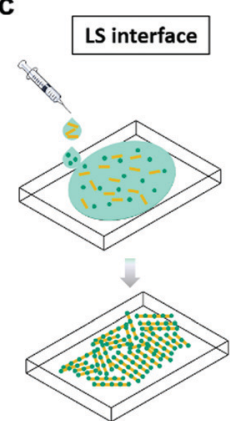

Fig. 11 The interfacial strategies for the synthesis of 2D c-MOF films. (a and b) Schematic illustration of gas-liquid and liquid-liquid interfacial methods. (c) Schematic illustration of liquid-solid interfacial synthesis.

liquid-solid (LS) interfacial approaches have been developed to prepare large-area thin 2D $c$-MOF films (Fig. 11). ${ }^{21,152-154}$

One representative work on the synthesis of $2 \mathrm{D} c$-MOF films was reported initially by Kambe et al. in 2013 using a LL interfacial strategy (Fig. 12a). ${ }^{74,75}$ An organic phase containing HTB in dichloromethane (DCM) was overlaid with an aqueous solution of $\mathrm{Ni}(\mathrm{OAc})_{2}$ and $\mathrm{NaBr}$ under an argon atmosphere. The water/DCM interface allowed the formation of a 1-2 $\mu \mathrm{m}$ thick $\mathrm{Ni}_{3}(\mathrm{HTB})_{2} \quad 2 \mathrm{D} \quad c$-MOF film with a lateral size of $\sim 100 \mu \mathrm{m}$ (Fig. 12b). PXRD and TEM analysis (Fig. 12c) indicated a crystal model of $\mathrm{Ni}_{3}(\mathrm{HTB})_{2}$ with parameters of $a=b=14.1 \AA$ and $c=7.6 \AA$ A. The same group also reported an isostructural $\mathrm{Pd}_{3}(\mathrm{HTB})_{2}$ using the above LL interfacial method. Additionally, $\mathrm{K}_{3}\left[\mathrm{Fe}(\mathrm{CN})_{6}\right]$ was added as a redox buffer to prevent the reduction
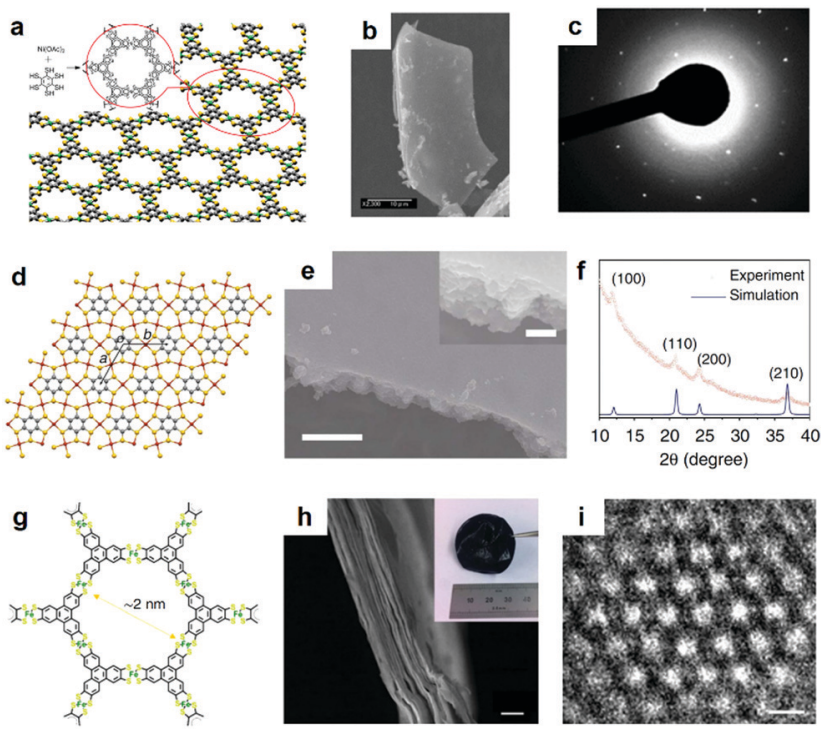

Fig. 12 Selected 2D c-MOFs synthesized through the LL interfacial method. (a-c) $\mathrm{Ni}_{3}(\mathrm{HTB})_{2} 2 \mathrm{D}$ c-MOF and its SEM and HR-TEM images. Reproduced with permission ref. 74. Copyright 2013, American Chemical Society. $(d-f)$ Lattice structure of $\mathrm{Cu}_{3}(\mathrm{HTB}) 2 \mathrm{D}$ c-MOF and its SEM image as well as in-plane GIXRD pattern. ${ }^{31}$ Copyright 2015, Nature Publishing Group. ( $\mathrm{g}-\mathrm{i}) \mathrm{Fe}_{3}(\mathrm{HTTP})_{2} 2 \mathrm{D} \mathrm{c}-\mathrm{MOF}$ and its SEM image from cross-side view and HR-TEM image. ${ }^{78}$ Copyright 2018, Nature Publishing Group. of $\mathrm{Pd}^{+}$to the formation of $\mathrm{Pd}^{0}{ }^{65}$ In 2015, Huang et al. reported a non-porous $2 \mathrm{D} c$-MOF network $\mathrm{Cu}_{3}(\mathrm{HTB})$ (Fig. $12 \mathrm{~d}-\mathrm{f}$ ) by coordination of HTB and $\mathrm{Cu}\left(\mathrm{NO}_{3}\right)_{2}$ at the water/DCM interface. ${ }^{31}$ The $\mathrm{Cu}_{3}$ (HTB) 2D $c$-MOF was obtained as a continuous film with a lateral size larger than $1 \mathrm{~cm}$ and thickness in the range of 20 to $140 \mathrm{~nm}$. In the $\mathrm{Cu}_{3}(\mathrm{HTB})$ network, each sulfur atom is coordinated to two copper atoms leading to a non-porous structure with denser ligands and metals. SEM and atomic force microscopy (AFM) images showed a continuously smooth upside in the film whilst the downside composed of plate-like nanosheets was fairly rough, especially for the thick films. The authors suggested that the water/DCM interface functioned initially as a platform for the formation of 2D $c$-MOF thin films (the upside in the final film), while the HTB ligand functioned as the transporter of the Cu-ions from the interface to the DCM phase below the interface. Then, the Cu-ions coordinated with the HTB molecules below the interface in a less order due to the weaker interface-confining effect here than that at the interface. Grazing incident X-ray diffraction (GIXRD) revealed a face-on orientation of $\mathrm{Cu}_{3}(\mathrm{HTB})$ in a $60 \mathrm{~nm}$ thick film whilst no preferred orientation was observed for the sample thicker than $200 \mathrm{~nm}$, indicative of randomly accumulated nanocrystals on the downside of the film near the water/DCM interface. Lahiri et al. also employed GL/LL interfacial methods for the construction of $\mathrm{M}_{3}(\mathrm{HIB})_{2}(\mathrm{M}=\mathrm{Co}, \mathrm{Ni}, \mathrm{Cu})$ thin and thick films with thicknesses of 10-13 nm and $\sim 1 \mu \mathrm{m}$, respectively. ${ }^{135}$

In 2016, Wu et al. reported a modified solution synthesis of large-area $\mathrm{Ni}_{3}(\mathrm{HITP})_{2}$ films in a homogeneous aqueous solution of HATP and $\mathrm{NiCl}_{2}$ using trimethylamine as the base under no perturbation. ${ }^{29}$ Upon heating the mixture to $60{ }^{\circ} \mathrm{C}, \mathrm{Ni}_{3}(\mathrm{HITP})_{2}$ nanoparticles self-assembled at the air/water interface and packed further closely to afford a continuous smooth film. The film thickness could reach $\sim 100 \mathrm{~nm}$ after $3 \mathrm{~min}$ of polymerization. In 2018, our group demonstrated a LL interfacial synthesis of the $\mathrm{Fe}_{3}(\mathrm{HTTP})_{2} 2 \mathrm{D} c$-MOF film (Fig. 12g). ${ }^{78}$ Organic salt of iron(II) acetylacetonate was dissolved in chloroform, which was overlaid by an aqueous phase containing the ligand HTTP and ammonia. Large-area $\left(\mathrm{cm}^{2}\right)$, free-standing $\mathrm{Fe}_{3}(\mathrm{HTTP})_{2}$ films were obtained with a tunable thickness from $20 \mathrm{~nm}$ to $\sim 2 \mu \mathrm{m}$ by increasing the reaction time (Fig. $12 \mathrm{~h}$ and i). TEM imaging revealed that the samples comprised of crystalline domains of ten to hundreds of $\mathrm{nm}$. A honeycomb structure with a pore size of $\sim 1.9 \mathrm{~nm}$ was observed by high-resolution TEM. PXRD displayed (100) and (200) peaks at $2 \theta=4.5^{\circ}$ and $9.1^{\circ}$, respectively, which demonstrated a honeycomb packing within the ab planes. The (001) reflection (peak at $2 \theta=27.3^{\circ}$ ) suggested an ordered stacking along the c direction with an interlayer distance of $\sim 0.33 \mathrm{~nm}$. Experimental PXRD patterns agreed well with the simulated inclined AA stacking models. The BET SSA was measured to be $\sim 526 \mathrm{~m}^{2} \mathrm{~g}^{-1}$. In 2019, the above $2 \mathrm{D} c$-MOF film with the thickness ranged from $\sim 84$ to $\sim 410 \mathrm{~nm}$ was also reported by Clough et al. using an ethyl acetate (containing 1\% NMP)/water interface. ${ }^{80}$

Apart from GL and LL approaches, LS interface-assisted synthetic methodology (also termed as on-substrate synthesis, Fig. 11c) has recently emerged for preparing $2 \mathrm{D} c$-MOF films 
with a preferred face-on orientation, for example, for HHTPbased systems. ${ }^{94,96,155,156}$ The preliminary on-substrate synthesis was explored by Sheberla et al. by placing a quartz substrate in the reaction mixture for the synthesis of bulk $\mathrm{Ni}_{3}(\mathrm{HITP})_{2} 2 \mathrm{D}$ $c$-MOFs. ${ }^{57} \mathrm{Ni}_{3}(\mathrm{HITP})_{2}$ was obtained as a $\sim 500 \mathrm{~nm}$ thick crystallite-accumulated macroscopic film on quartz surface. In 2017, Yao et al. reported a spray layer-by-layer liquid-phase epitaxial method for preparing face-on orientated $\mathrm{Cu}_{3}(\mathrm{HHTP})_{2}$ thin films. ${ }^{96}$ Typically, $-\mathrm{OH}$ functionalized substrates were alternatively exposed to a solution of HHTP and copper(II) acetate in ethanol by a spray method (Fig. 13a and b). The thickness could be controlled from 20 to $100 \mathrm{~nm}$ with an average increment of $\sim 2 \mathrm{~nm}$ after each growing cycle. Topand side-view SEM images reveal a dense and continuous thin film with a moderately smooth surface on the substrate (Fig. 13c). Later on, Mahringer et al. explored a vapor-assisted conversion $^{106}$ as an effective way for the synthesis of face-on oriented $\mathrm{Cu}_{3}(\mathrm{HHTP})_{2}$ and $\mathrm{M}_{9}(\mathrm{HHTP})_{4}(\mathrm{M}=\mathrm{Ni}, \mathrm{Co})$ thin films on various substrates. ${ }^{94}$ The authors utilized an additive of 1-propanol as a modulator for enlarging the crystal size (up to $10 \mu \mathrm{m}$ for $\mathrm{Cu}_{3}(\mathrm{HHTP})_{2}, 1-2 \mu \mathrm{m}$ for $\mathrm{Ni}_{9}(\mathrm{HHTP})_{4}$, and 3-4 $\mu \mathrm{m}$ for $\left.\mathrm{Co}_{9}(\mathrm{HHTP})_{4}\right)$. In a typical synthesis, a water/1-propanol $(\mathrm{v} / \mathrm{v}=1 / 1)$ solution of precursor was spread onto an elevated gold substrate in a reaction vessel containing the vapor source of the same water/1-isopropnaol mixture, and heated at $85{ }^{\circ} \mathrm{C}$ for $3 \mathrm{~h}$ (Fig. 13d). GIXRD patterns in the in-plane and out-of-plane directions indicated a preferential orientation of $\mathrm{Cu}_{3}(\text { HHTP })_{2}$ or $\mathrm{M}_{9}(\text { HHTP })_{4}$ layers with stacking reflections aligned orthogonally to the surface of the substrates. These samples are free of crack over a large area with thicknesses of $\sim 200 \mathrm{~nm}$ (Fig. 13e). Cross-section SEM images revealed packed pillared crystallites, corresponding to the observation of GIXRD analysis. The LS interfacial synthesis of high quality thin films allows direct device fabrication by adopting conductive or insulating substrates for electronic devices. ${ }^{94,96,97}$
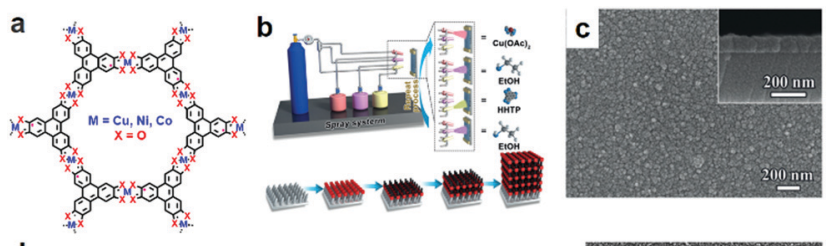

d
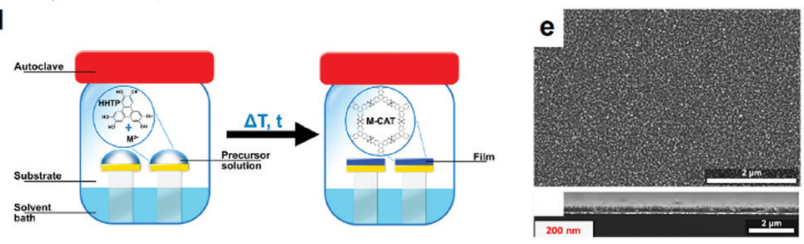

Fig. 13 Oriented HHTP-based 2D c-MOF films synthesized through various LS interfacial methods. (a) HHTP-based 2D c-MOFs. (b and c) Schematic illustration of the spray layer-by-layer liquid-phase epitaxial method and the SEM image of the obtained $\mathrm{Cu}_{3}(\mathrm{HHTP})_{2}$ film. Reproduced with permission ref. 96. Copyright 2017, Wiley- $\mathrm{VCH}$. (d and e) Schematic illustration of the vapor-assisted conversion method and the top-/sideview SEM images. Reproduced with permission ref. 94. Copyright 2019, American Chemical Society.
Apart from 2D c-MOF thin/thick films and thin nanosheets, the construction of a single-layer sample with high structural control at the atomic level has remained a synthetic challenge. The single-layer sample will offer distinguished properties due to the 2D material nature, ${ }^{157-159}$ which include, but are not limited to size effects on the intrinsic properties, varied electronic and optical properties due to the absence of interlayer interactions, high exposure of surface active sites, high mechanical flexibility, facile device design and processing, and further construction of multicomponent van der Waals heterostructures. ${ }^{45,61,98,160}$ In 2015 , our group reported the preparation of the first example of large-area, free-standing, single-layer 2D $c$-MOFs through Langmuir-Blodgett (LB) assisted interfacial synthesis (Fig. 14), ${ }^{161}$ by controlling the surface pressure (Fig. 15a and b). ${ }^{45}$

Typically, to produce a single-layer $\mathrm{Ni}_{3}(\mathrm{HTTP})_{2}$, a mixed solution of ligand HTTP and DMF/chloroform $(v / v=1 / 3)$ was spread over the water surface in a LB trough. Subsequently, the HTTP molecules were compressed into a dense self-assembled monolayer through monitoring and increasing the surface pressure to $10 \mathrm{mN} \mathrm{m}^{-1}$, and an aqueous solution of $\mathrm{Ni}\left(\mathrm{NO}_{3}\right)_{2}$ was injected into the water phase. With the diffusion of the $\mathrm{Ni}^{2+}$ ions to the air/water interface, a coordination reaction was triggered between HTTP and $\mathrm{Ni}^{2+}$ ions. A free-standing singlelayer $\mathrm{Ni}_{3}$ (HTTP $)_{2}$ film was obtained and could be directly transferred onto various substrates for further characterization. The thickness of the film was measured to be $\sim 0.7 \mathrm{~nm}$ by AFM (Fig. 15c and d). As the single-layer sheets showed low stability under electron irradiation, selected area electron diffraction (SAED) was performed by cryogenic TEM at $-175^{\circ} \mathrm{C}$, exhibiting a typical hexagonal diffraction pattern. This SAED result at least suggested local crystallinity presenting in the single-layer $2 \mathrm{D}$ $c$-MOF with an ordered honeycomb network having a cell size of $\sim 2 \mathrm{~nm}$. Such a $\mathrm{Ni}_{3}(\mathrm{HTTP})_{2}$ 2D $c$-MOF was transferred onto glassy carbon electrodes for electrocatalytic water splitting. Currently, due to their well-defined structure and abundant active sites as well as intrinsic conductivity, 2D $c$-MOFs have been rising as a promising electrode material for electrochemical energy devices.

These efforts from various interfacial approaches allow the integration of 2D $c$-MOFs into functional devices and thus exploring their multifunctional properties. However, the

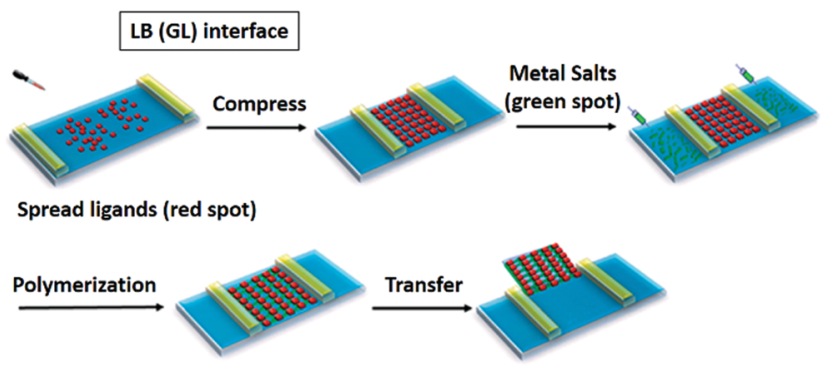

Fig. 14 Schematic illustration of Langmuir-Blodgett air/water (a special $\mathrm{GL}$ interface) interface-assisted synthesis. Reproduced with permission ref. 21. Copyright 2018, American Chemical Society. 
a

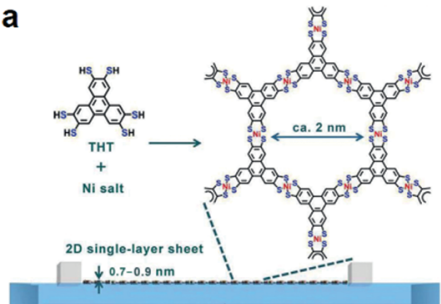

C

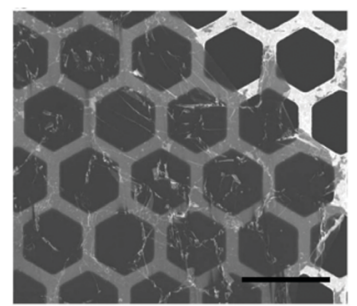

b

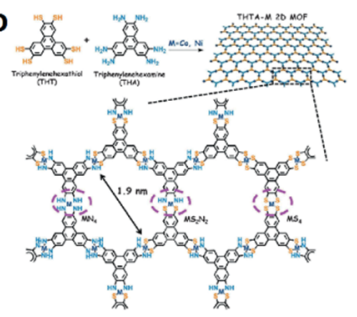

d

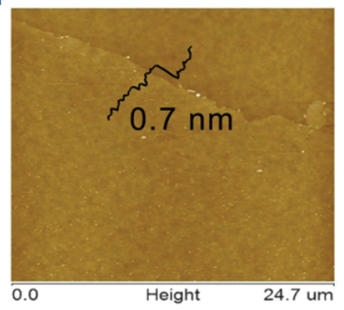

a

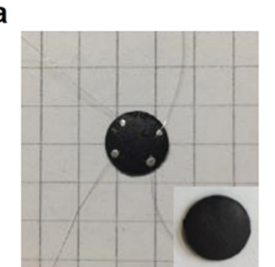

C

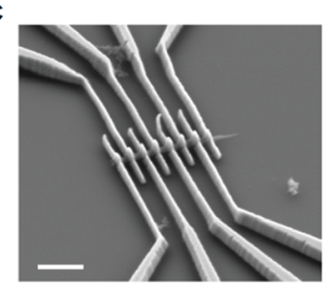

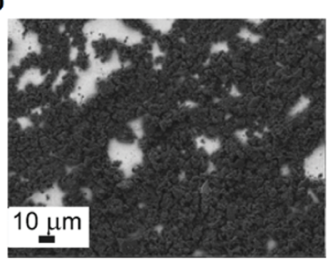

d

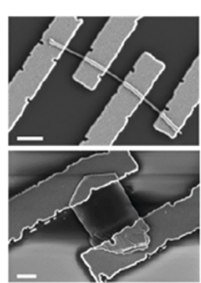

Fig. 16 Device integration of bulk 2D c-MOFs. (a) Pellet of 2D c-MOFs and device fabrication in the van der Pauw geometry. ${ }^{55}$ Copyright 2020 , Wiley-VCH. (b) SEM image of drop-cast $\mathrm{Cu}_{3}(\mathrm{HITP})_{2}$ on an indium-tin-oxide glass slide. Reproduced with permission ref. 28. Copyright 2015, Wiley$\mathrm{VCH}$. (c) SEM image of the fabricated device based on isolated singlecrystalline $\mathrm{Ni}_{3}(\mathrm{HITP})_{2}$ nanorods. (d) SEM images of fabricated devices based on isolated single-crystalline $\mathrm{Cu}_{3}(\mathrm{HHTP})_{2}$ nanorods (top) and nanoflakes (down). Reproduced with permission ref. 95. Copyright 2019, American Chemical Society.

interfacial strategies have been so far rather limited; the successful synthesis strongly relied on the association of high solubility of selective building blocks in water or organic solvents. In addition, accessibility on controlling/tuning orientation remains to be explored. In addition to wet-surface, the on-surface synthesis using $\mathrm{Cu}(111)$ or $\mathrm{Au}(111)$ substrates under ultrahigh vacuum provides another pathway to develop a single-layer 2D $c$-MOF that guarantees a molecular structural resolution with the support of a scanning tunneling microscope (STM). ${ }^{162-165}$ In 2018, Lischka et al. reported a topologically diverse framework upon annealing HATP on $\mathrm{Cu}(111)$. A metalorganic trimer composed of three ligand molecules linked by a $\mathrm{Cu}_{3}$ cluster instead of a single $\mathrm{Cu}$ atom was observed, while ring-fused $\mathrm{Ni}_{3}(\mathrm{HITP})_{2}$ with a limited number of repeating units appeared in the presence of additional $\mathrm{Ni}$ on $\mathrm{Cu}(111) .{ }^{163}$ Later on, by varying $\mathrm{Cu}(111)$ surface to $\mathrm{Au}(111)$, Gao et al. obtained a monolayer of $\mathrm{Ni}_{3}(\mathrm{HITP})_{2}$ with a domain size of $\sim 40 \mathrm{~nm}$ and resolved its structure at sub-molecular level by STM. ${ }^{162}$ Recently, Zhang et al. applied the on-surface method for bonding $\mathrm{HHB}$ with $\mathrm{Cu}$ atoms; however, a single-layer 2D nonconjugated MOF was determined. ${ }^{165}$ Despite the preliminary success of the on-surface synthesis, many synthetic challenges remain to be addressed, such as the synthesis of large-area (from $\mu \mathrm{m}^{2}$ to $\mathrm{cm}^{2}$ ), highly-ordered monolayer networks, the complete lift of the monolayer from the solid surface for device integration, and so on.

\section{Device integration based on multi- dispersed 2D c-MOFs}

As is shown, 2D $c$-MOFs have been synthesized by various synthetic methodologies and have shown diverse morphologies,

such as bulk powder (polycrystalline nanoparticles or singlecrystals) and large-area thin films. Regarding the bulk powders, compressed pellets (Fig. 16a) have been widely employed for device integration (other method e.g. drop-casting, Fig. 16b) and charge transport characterization. Nonetheless, these pellet samples are generally comprised of nanocrystals with no preferred orientation, co-existing with numerous grain boundaries and defects, ${ }^{166}$ which limited the conductivity performance and the unambiguous understanding on the transport mechanism. By comparison, the single-crystalline 2D c-MOF samples offer the opportunity for device investigation based on each crystal (nanorods or nanoflakes) and allow precise and reliable analysis of the intrinsic electronic properties (Fig. 16c and d). However, the isolated single-crystalline nanorod or nanoflake sample suffers from synthetic challenges and limitation in practical functional studies.

In regard to addressing the above challenges with respect to application of the bulk crystals (polycrystals and single-crystals) obtained by the hydro-/solvothermal syntheses, top-down exfoliation through methods such as ball-milling ${ }^{167,168}$ and sonication $^{144,145,169}$ (Fig. 17a) is expected to be an efficient approach to achieve thin nanosheets, ${ }^{169-172}$ which can maintain the intrinsic conductivity and porosity, allow high exposure of active sites, as well as facile solution-processible device integration. ${ }^{173}$ Currently, few top-down methods have been applied to the delamination of bulk 2D $c$-MOFs.

Very recently, we employed ball milling exfoliation for the synthesis of $\mathrm{Ni}_{2}\left[\mathrm{CuPc}(\mathrm{NH})_{8}\right] 2 \mathrm{D} c$-MOF nanosheets. ${ }^{55}$ Firstly, a highly crystalline bulk $\mathrm{Ni}_{2}\left[\mathrm{CuPc}(\mathrm{NH})_{8}\right]$ sample was prepared with an average domain size of $\sim 200 \mathrm{~nm}$ via a solvothermal protocol. Then, via a NaCl-assisted low energy ball milling strategy (Fig. 18a), bulk $\mathrm{Ni}_{2}\left[\mathrm{CuPc}(\mathrm{NH})_{8}\right]$ nanocrystals were 


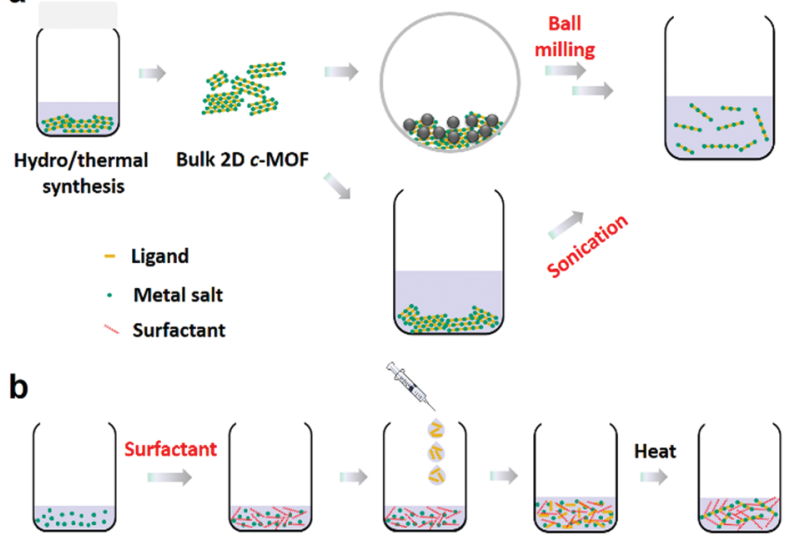

Fig. 17 Schematic illustration of the synthesis of 2D c-MOF nanosheets through top-down and bottom-up method. (a) Top-down ball milling and sonication exfoliation. (b) Bottom-up surfactant-assisted synthesis.

mechanically exfoliated into nanosheets with a yield of $40-50 \%$. PXRD patterns revealed a shift of the layer distance from 3.24 to $3.33 \AA$, indicative of swollen layers after milling. SEM, AFM and HR-TEM together indicated an average lateral size of $\sim 160 \mathrm{~nm}$ and a thickness of $\sim 7 \mathrm{~nm}$ for these nanosheets (Fig. 18b). The achieved nanosheets could be homogeneously dispersed in DMF for more than six months that allows facile solutionprocessible micro-supercapacitor device fabrication through filtration (further discussion is in Section 6.5.2).

Even though the top-down exfoliation method offers a possibility in scalable synthesis of nanosheets with accessible active sites and solution-processibility, it remains limited in the control of the morphology, the layer numbers and the lateral domain size. By contrast, bottom-up methods that refer to the direct synthesis of the nanosheets from metal nodes and ligands hold promise in the synthesis of highquality 2D c-MOF nanosheets. Recently, our group introduced a surfactant-mediated synthetic method toward single-crystalline $\mathrm{Cu}_{3}(\mathrm{HHB})_{2}$ nanosheets (Fig. 17b). ${ }^{88}$ The sodium dodecyl sulfate

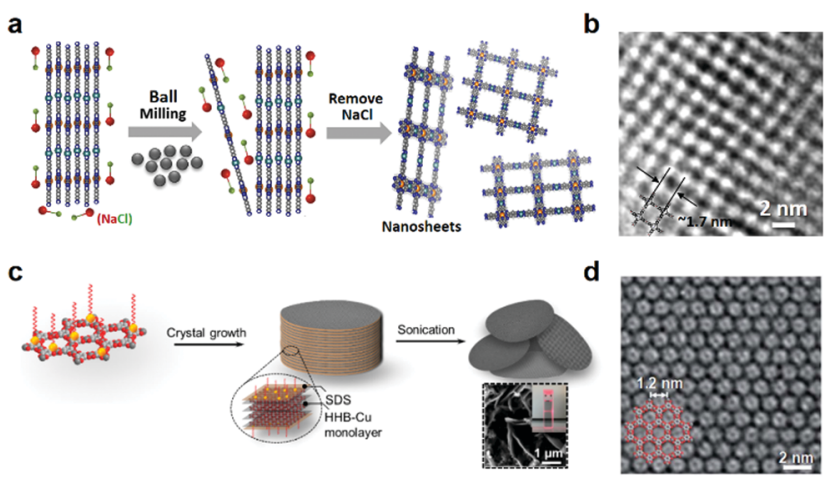

Fig. 18 Synthesis of 2D c-MOF nanosheets. (a and b) Schematic illustration of the $\mathrm{NaCl}$-assisted ball-milling exfoliation of $\mathrm{Ni}_{2}\left[\mathrm{CuPc}(\mathrm{NH})_{8}\right]$ and HR-TEM image of the synthesized nanosheets. ${ }^{55}$ Copyright 2020 , Wiley$\mathrm{VCH}$. (c and d) Schematic illustration of surfactant-assisted synthesis of $\mathrm{Cu}_{3}(\mathrm{HHB})_{2} 2 \mathrm{D} \mathrm{c}-\mathrm{MOF}$ nanosheets and the related HR-TEM image. ${ }^{88}$ Copyright 2020, the Royal Society of Chemistry.
(SDS) anionic surfactant was used as the directing agent for restricting the growth of $2 \mathrm{D} c$-MOFs along the $\mathrm{c}$ direction and weakening the interlayer interactions. ${ }^{174,175}$ In a typical surfactant-mediated hydrothermal synthesis, the precursors of THQ, $\mathrm{Cu}(\mathrm{OAc})_{2}$, and $\mathrm{NaOH}$ (base) in a SDS aqueous solution were ultrasonicated at $50{ }^{\circ} \mathrm{C}$ to prepare the nanosheets with an ultrahigh yield of $80-90 \%$ (Fig. 18c). As a consequence, the achieved nanosheets presented a thickness of $4.2 \pm 1.1 \mathrm{~nm}$ and a lateral size of $0.30-0.65 \mu \mathrm{m}^{2}$, as well as a much higher BET SSA $\left(385 \mathrm{~m}^{2} \mathrm{~g}^{-1}\right)$ than the contrast powder sample $\left(119 \mathrm{~m}^{2} \mathrm{~g}^{-1}\right)$ obtained in the absence of SDS. HR-TEM and related SAED analysis demonstrated the achievement of single-crystalline nanosheets without noticeable defects (Fig. 18d). The high exposure of active sites in the nanosheet sample allows fast ion/electron diffusion hence ensuring superior performance than the related bulk $\mathrm{Cu}_{3}(\mathrm{HHB})_{2}$ as an electrode material (Section 6.5.1).

Synthesis of solution-processible nanosheets will be facile for the fabrication of 2D $c$-MOF based devices via spin-coating, filtration, ink-printing, etc. In this respect, it requires to address the key questions on the homogeneous dispersion in solution and the control of size, thickness and crystallinity as well as avoiding the re-stacking during solidification. Definitely, another choice is to utilize the free-standing, large-area, highly crystalline 2D $c$-MOF thin films with tunable thickness, which will simplify the device fabrication by direct deposition onto the substrates. To this end, an extraordinary property and a reliable structure-property relationship are expected, thus pushing the functional development for MOFtronics.

\section{Functions for MOFtronics}

Conductive 2D c-MOFs possess a series of unique features, such as large surface to volume ratio, defined active sites, and tailored electronic structure that enables a set of intriguing properties from semiconductor and redox activity to metallicity, ferromagnetism, photoswitching, etc. High conductivities in 2D $c$-MOFs have attracted great attention in various MOFtronics including (opto)electronics, spintronics, and thermoelectrics, as well as energy storage devices as potential power sources for MOFtronics.

\subsection{Electronics}

The emerging 2D $c$-MOFs featuring semiconductor properties, high conductivity, and electrically-transduced sensing have been expected to be outstanding substitutes for potential applications in electronics, such as field-effect transistors, superconductors, chemiresistors, etc.

6.1.1 Field-effect transistors (FETs). FETs are a class of three-terminal semiconductor electronic devices with high integration and mass production ${ }^{29}$ that have played key roles in the current logic and memory devices and sensors. In addition, FETs represent an efficient tool for the study of charge transport properties including the type of charge carries and their mobility values. In 2015, Huang et al. fabricated a FET based on the $\mathrm{Cu}_{3}(\mathrm{HTB})$ film in a bottom-gate bottom-contact 

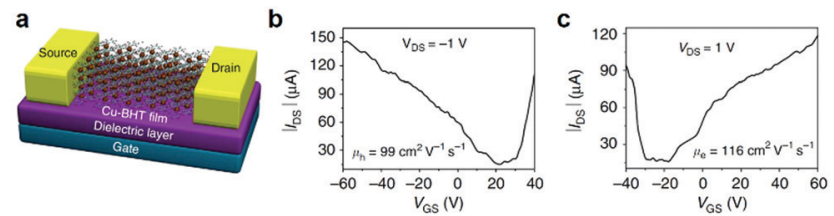

d
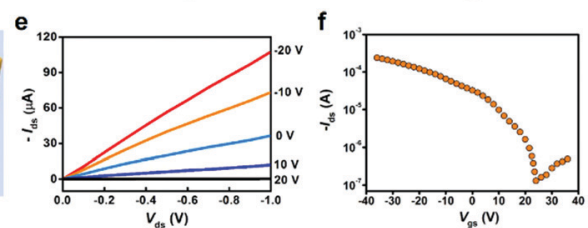

Fig. 19 2D c-MOF based FET devices. (a-c) Illustrative schematic of FET based on $\mathrm{Cu}_{3}(\mathrm{HTB})$ and related transfer characteristics. ${ }^{31}$ Copyright 2015 , Nature Publishing Group. (d) $\mathrm{Ni}_{3}(\mathrm{HITP})_{2}$-based FET. (e and f) Output curves and transfer characteristic plot of $\mathrm{Ni}_{3}(\mathrm{HITP})_{2}$, respectively. Reproduced with permission ref. 29. Copyright 2017, American Chemical Society.

geometry (Fig. 19a). ${ }^{31}$ The drain-source current $\left(I_{\mathrm{ds}}\right)$ versus gatesource voltage $\left(V_{\mathrm{gs}}\right)$ curves indicated ambipolar behavior with outstanding hole and electron mobility of 99 and $116 \mathrm{~cm}^{2} \mathrm{~V}^{-1} \mathrm{~s}^{-1}$ respectively as well as a current on/off ratio of 10 (Fig. 19b and c). The low on/off ratio correlates with the metallic nature of $\mathrm{Cu}_{3}(\mathrm{HTB})$. Later on, Wu et al. applied the $\mathrm{Ni}_{3}(\mathrm{HITP})_{2} 2 \mathrm{D} c$-MOF film in a bottom-gate top-contact geometry (Fig. 19d). ${ }^{29}$ The curve of $I_{\mathrm{ds}}$ as a function of $V_{\mathrm{gs}}$ showed an increased channel current with a declined $V_{\mathrm{gs}}$, suggestive of a p-type semiconducting behavior for the as-synthesized $\mathrm{Ni}_{3}$ (HITP $)_{2}$ film sample with a mobility up to $48.6 \mathrm{~cm}^{2} \mathrm{~V}^{-1} \mathrm{~s}^{-1}$ (holes as the majority of carriers) and a high current on/off ratio of $\sim 2000$ (Fig. 19e and f). Recently, Wang et al. integrated the $\mathrm{Ni}_{3}(\mathrm{HITP})_{2}$ film synthesized by the LS interfacial method into FETs with bottom-contact device geometry. ${ }^{107}$ The FET showed similar performance to the previous report, with a mobility of $45.4 \mathrm{~cm}^{2} \mathrm{~V}^{-1} \mathrm{~s}^{-1}$. Using the FET as a liquid-gated device, this $2 \mathrm{D} c$-MOF was further explored in FET-based biosensors.

The porous and tailored structure of $2 \mathrm{D} c$-MOFs ensures the diversity of their FET devices. However, many challenges remain to date, such as the formation of the $2 \mathrm{D} c$-MOF film with high smoothness and controllable thickness, tunability of band gaps, optimized integration for good contacts, etc.

6.1.2 Superconductors. The zero resistance and the Meissner effect characterizing superconductivity has been considered as an important parameter in power transmission, NMR imaging, maglev, and superconducting quantum interference devices (SQUIDs). ${ }^{68} \mathrm{Cu}_{3}$ (HTB) exhibiting a zero band gap and extraordinary conductivity could function as an excellent material candidate for this application..$^{31,68,126}$ Through electrical resistivity, AC magnetic susceptibility, and specific heat measurements, Zhu and co-workers detected a superconducting transition at $0.25 \mathrm{~K}$ for $\mathrm{Cu}_{3}$ (HTB) (Fig. 20a), which remains the only example in this field. The transition depended on the magnetic field and disappeared at an applied field of 2500 Oe (Fig. 20b). Moreover, a second superconductivity phase with a transition temperature of $\sim 3 \mathrm{~K}$ in the magnetic susceptibility measurement was determined and it is ascribed to the superconductivity of monolayers or few-layers of $\mathrm{Cu}_{3}(\mathrm{HTB})$.
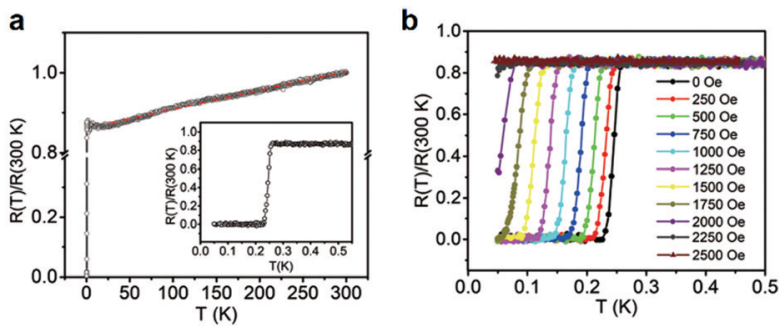

Fig. 20 Superconductivity of $\mathrm{Cu}_{3}(\mathrm{HTB})$. (a) Temperature-dependence of the normalized resistance from 0.05 to $300 \mathrm{~K}$ of $\mathrm{Cu}_{3}(\mathrm{HTB})$. (b) Temperature-dependence of the normalized resistance of $\mathrm{Cu}_{3}(\mathrm{HTB})$ in applied magnetic fields up to 2500 Oe. Reproduced with permission ref. 68. Copyright 2018, Wiley-VCH.

This observation is in accordance with the calculated results; the monolayer of $\mathrm{Cu}_{3}(\mathrm{HTB})$ has a predicted transition temperature of $4.43 \mathrm{~K}$, while it decreased to $1.58 \mathrm{~K}$ for the bulk one. ${ }^{126}$

6.1.3 Chemiresistors. Gas sensors are desirable for disease diagnosis and prevention as well as environmental protection. ${ }^{24}$ Benefiting from the large surface to volume ratio, MOFs are able to serve as active materials. Nonetheless, due to the lack of intrinsic electrical conductivity in traditional MOFs, the fabricated sensors have been limited to devices with a complicated transducing mechanism such as luminescence quenching or enhancement. ${ }^{28}$ Conductive $2 \mathrm{D} c$-MOFs hold considerable promise for fast signal transduction in chemiresistive sensors, which represent one of the most intensively studied MOFtronics. The preliminary results reported to date indicate the immerse potential of these materials for efficient detection of various gas analytes.

2D $c$-MOF based chemiresistors were demonstrated by

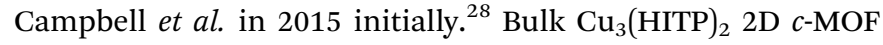
was transferred onto interdigitated gold electrodes by dropcasting an acetone dispersion of $\mathrm{Cu}_{3}(\mathrm{HITP})_{2}$ to construct the device. The chemiresistor was capable of detection of $\mathrm{NH}_{3}$ in parts per million ( $\mathrm{ppm}$ ) concentration level at room temperature, in spite of the existence of $60 \%$ relative humidity (RH) (Fig. 21a and b). However, the isostructural $\mathrm{Ni}_{3}(\mathrm{HITP})_{2} 2 \mathrm{D} c$ MOF did not produce any reliable response under identical conditions, indicating the impact of rational modification on metal nodes on the functionality. Later on, Mirica's group reported the utilization of $\mathrm{Cu}_{3}(\mathrm{HHTP})_{2}$ and $\mathrm{M}_{9}(\mathrm{HHTP})_{4}$ $(\mathrm{M}=\mathrm{Ni}, \mathrm{Co}) 2 \mathrm{D} c$-MOFs for the detection of $\mathrm{NH}_{3}$ by preparing the $2 \mathrm{D} c$-MOF/graphite hybrids ${ }^{92}$ or assembling the $2 \mathrm{D} c$-MOFs on graphitic electrodes ${ }^{176}$ through the typical hydrothermal method. In 2018, combining experiment with simulation, Rubio-Gimenez et al. ascribed the origin of response of $\mathrm{Cu}_{3}(\mathrm{HHTP})_{2}$ toward $\mathrm{NH}_{3}$ to the coordination of $\mathrm{NH}_{3}$ to $\mathrm{Cu}^{2+}$ in the network. ${ }^{122}$ Kelvin probe measurements revealed the difference of work function after exposing $\mathrm{Cu}_{3}(\mathrm{HHTP})_{2}$ to $\mathrm{NH}_{3}$, and infrared (IR) reflection absorption spectroscopy indicated the appearance of $\mathrm{NH}_{3}$ stretching bands and the coordination between $\mathrm{NH}_{3}$ and $\mathrm{Cu}$ atom in $\mathrm{Cu}_{3}(\mathrm{HHTP})_{2}$. According to the simulation, the coordination leads to slight distortions of the electronic structure and triggers an increase in the band gap from 0.33 to $0.42 \mathrm{~V}$. Yao et al. integrated the $\mathrm{Cu}_{3}(\mathrm{HHTP})(\mathrm{HHB})$ 
a
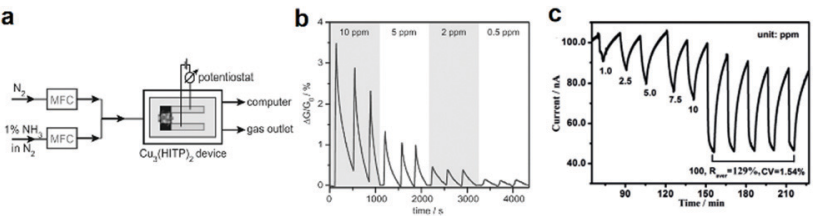

d
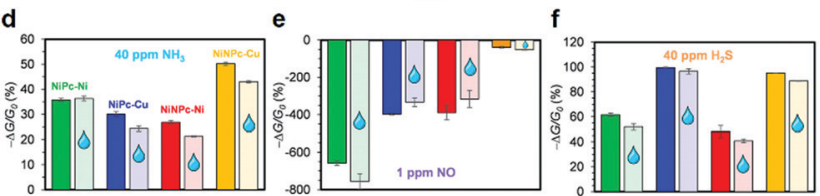

Fig. $212 \mathrm{D}$ c-MOF based chemiresistors for the detection of $\mathrm{NH}_{3}, \mathrm{NO}$ and $\mathrm{H}_{2} \mathrm{~S}$. ( $a$ and b) Schematic of the experimental apparatus and relative response curve of $\mathrm{Cu}_{3}(\mathrm{HITP})_{2}$ toward $\mathrm{NH}_{3}$ at different concentrations. Reproduced with permission ref. 28. Copyright 2015, Wiley-VCH. (c) The response-recovery curve of the $\mathrm{Cu}_{3}(\mathrm{HHTP})_{2}$ thin film toward $\mathrm{NH}_{3}$ at different concentrations. Reproduced with permission ref. 96. Copyright 2017, Wiley$\mathrm{VCH}$. (d-f) Sensing responses of $\mathrm{M}_{2}\left[\mathrm{NiPcO}_{8}\right]$ and $\mathrm{M}_{2}\left[\mathrm{NiNPCO}_{8}\right](\mathrm{M}=\mathrm{Cu}, \mathrm{Ni})$ upon 30 min exposure to $40 \mathrm{ppm}$ of $\mathrm{NH}_{3}, \mathrm{H}_{2} \mathrm{~S}$, and $1 \mathrm{ppm}$ of $\mathrm{NO}$ in dry nitrogen and in the presence of $\mathrm{H}_{2} \mathrm{O}$. Reproduced with permission ref. 51 . Copyright 2019, American Chemical Society.

2D $c$-MOF into a gas sensor by the drop-casting method which showed good sensing performance for $\mathrm{NH}_{3}$ in the concentration range of $1-100 \mathrm{ppm} .{ }^{59}$ IR spectroscopy and PXRD spectroscopy revealed the $\mathrm{NH}_{3}-\mathrm{Cu}^{+/ 2+}$ interaction with an expanded c lattice upon exposure to $\mathrm{NH}_{3}$. Important advancement was reported by $\mathrm{Xu}$ and co-workers using the high quality $\mathrm{Cu}_{3}(\mathrm{HHTP})_{2}$ thin film prepared through the LS interfacial approach. ${ }^{96}$ The resulting sensor exhibited a high response of $129 \%$ and $>10 \%$ toward 100 and $1 \mathrm{ppm}$ of $\mathrm{NH}_{3}$ vapor, respectively (Fig. 21c). The authors calculated the theoretical limit of detection (LOD) to be $\sim 0.5 \mathrm{ppm}$ by setting the response as $10 \%$. In addition, the response and recovery were accelerated by $54 \%$ and $10 \%$ respectively compared with those of the corresponding bulk $\mathrm{Cu}_{3}(\mathrm{HHTP})_{2}$ based sensor.

Besides $\mathrm{NH}_{3}$, Mirica and co-workers demonstrated $\mathrm{Cu}_{3}(\mathrm{HHTP})_{2}$ and $\mathrm{Ni}_{9}$ (HHTP $)_{4} 2 \mathrm{D} c$-MOFs as the active materials for the chemiresistive response to other toxic gases including NO and $\mathrm{H}_{2} \mathrm{~S} .{ }^{176}$ The same group integrated $\mathrm{Ni}_{9}(\mathrm{HHTP})_{4}$ or $\mathrm{Ni}_{3}(\mathrm{HITP})_{2}$ into textile-supported gas sensors for the detection of $\mathrm{NO}$ and $\mathrm{H}_{2} \mathrm{~S}$, which exhibited good mechanical flexibility. ${ }^{102}$ The sensors were able to detect $\mathrm{NO}$ and $\mathrm{H}_{2} \mathrm{~S}$ with high performance in the presence of humidity $(18 \% \mathrm{RH})$ and were fully recoverable after washing the devices with water. Recently, Meng et al. reported chemiresistive sensors based on a series of phthalocyanine derivatives-based $2 \mathrm{D} c$-MOFs $\left(\mathrm{M}_{2}\left[\mathrm{NiPcO}_{8}\right]\right.$ and $\left.\mathrm{M}_{2}\left[\mathrm{NiNPcO}_{8}\right], \mathrm{M}=\mathrm{Cu}, \mathrm{Ni}\right){ }^{51}$ The resulting devices displayed outstanding humidity-independent ability during the detection and differentiation of $\mathrm{NH}_{3}, \mathrm{H}_{2} \mathrm{~S}$, and $\mathrm{NO}$, and delivered sub-ppm or part-per-billion (ppb) level theoretical LODs at room temperature (Fig. 21d-f).

In 2015, Campbell et al. contributed to a cross-reactive sensor array, which was capable of distinguishing five categories of volatile organic compounds (VOCs) (alcohols, amines, ketones/ethers, aromatic and aliphatic hydrocarbons) (Fig. 22a and b). ${ }^{91}$ Structurally analogous $\mathrm{Cu}_{3}(\mathrm{HHTP})_{2}$ and $\mathrm{M}_{3}(\mathrm{HITP})_{2}(\mathrm{M}=\mathrm{Cu}, \mathrm{Ni}) 2 \mathrm{D} c$-MOFs were applied for the
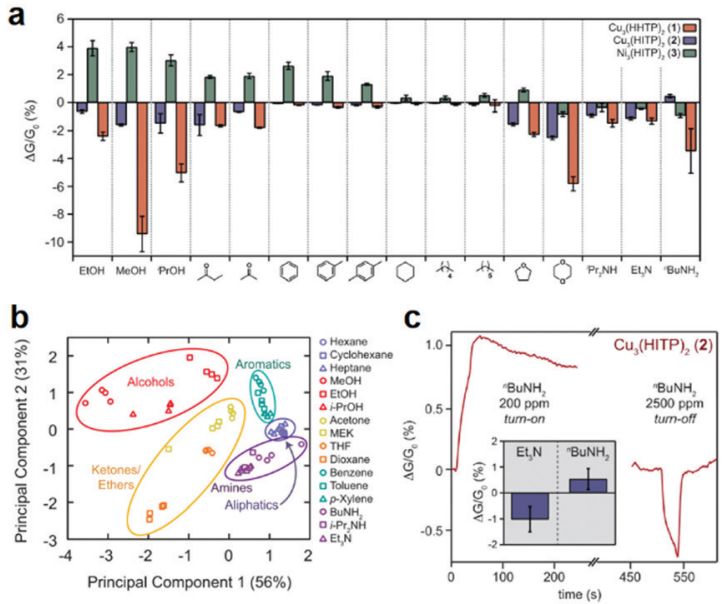

Fig. 22 2D c-MOF based chemiresistors for the detection of VOCs. (a and b) Sensing responses and related principal component analysis to various 200 ppm of VOC vapors for the 2D c-MOF array: $\mathrm{M}_{3}(\mathrm{HXTP})_{2}(\mathrm{M}=\mathrm{Cu}, \mathrm{Ni}$; $X=O, N H)$. (c) Sensing response for $\mathrm{Cu}_{3}(\mathrm{HITP})_{2}$ to ${ }^{n} \mathrm{BuNH}_{2}$. Reproduced with permission ref. 91. Copyright 2015, American Chemical Society.

fabrication of sensors through drop-casting. These $2 \mathrm{D} c$-MOF based devices exhibited diverse sensing behaviors toward various VOC vapors at a concentration of $200 \mathrm{ppm}$. Both varying the metal nodes and ligands led to changes in the response to each gas. Furthermore, all samples delivered a high response to polar VOCs, while no appreciable signals were recorded for the aliphatic hydrocarbons. The authors noted both "turn-on" and "turn-off" responses of $\mathrm{Cu}_{3}(\mathrm{HITP})_{2}$ for ${ }^{\mathrm{n}} \mathrm{BuNH}_{2}$ at 200 and $2500 \mathrm{ppm}$ respectively (Fig. 22c) and speculated a multiple sensing mechanism including charge transfer and hydrogen bonding among these 2D $c$-MOFs. Later on, Hoppe et al. prepared a 1-3 $\mu \mathrm{m}$ thick $\mathrm{Cu}_{3}(\mathrm{HHTP})_{2}$ film by spray-coating its dispersion on glass slides or flexible polycarbonate foils and fabricated chemiresistive sensors for methanol sensing accordingly. ${ }^{93}$

Besides conventional analytes, the groups of Mirica and Dincă have applied 2D $c$-MOFs for the detection of ions $\left(\mathrm{Cu}_{3}(\mathrm{HHTP})_{2} \text {, and } \mathrm{M}_{9}(\mathrm{HHTP})_{4}\right)^{177}$ and $\mathrm{CO}_{2} \quad\left(\mathrm{Cu}_{3}(\mathrm{HIB})_{2}\right){ }^{178}$ respectively. Despite numerous successful examples of $2 \mathrm{D}$ $c$-MOFs for sensors, the thus-far fabricated gas sensors through the compressing or drop-casting method based on bulk 2D $c$-MOFs obtained by solvothermal synthesis, have brought difficulties in realizing the mass production of devices and exhibited limited performance. Analogous to FETs, the rational tuning of the electronic and chemical structures, improving the quality of thin 2D $c$-MOF films, and further optimizing the integration methods thus leading to high performance remain to be explored. Moreover, fundamental understanding of the sensing mechanisms such as analyte-metal coordination, hydrogen bonding interaction, and guest-host interaction to ascertain neat structure-property relationship requires under investigation.

\subsection{Optoelectronics}

Conductive 2D $c$-MOFs with tailorable band gaps are appealing materials for optoelectronics, such as photodetectors, organic 
a
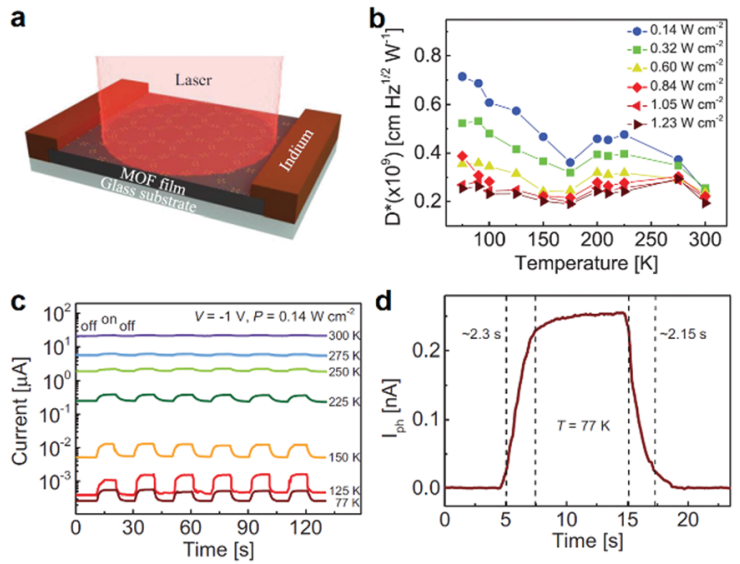

Fig. $23 \mathrm{Fe}_{3}(\mathrm{HTTP})_{2} 2 \mathrm{D}$ c-MOF based photodetector. (a and b) Schematic of a two-terminal photodetector device based on $1.7 \mu \mathrm{m}$ thick $\mathrm{Fe}_{3}(\mathrm{HTTP})_{2}$ and its dependence of detectivity at $-1 \mathrm{~V}$ bias. (c) Temperature-dependent photoresponse versus time. (d) Zoomed time-resolved response at $77 \mathrm{~K}$ showing a response time of $\sim 2 \mathrm{~s} .{ }^{30}$ Copyright 2020, Wiley- $\mathrm{VCH}$.

light-emitting devices, solar cells, etc. ${ }^{30,143,179}$ Very recently, we and cooperation partner demonstrated a $\mathrm{Fe}_{3}(\mathrm{HTTP})_{2}$ based twoterminal photodetector device operated in photoconductive mode (Fig. 23a). ${ }^{30}$ The record-high mobility and band-like charge transport of $\mathrm{Fe}_{3}(\mathrm{HTTP})_{2}$ allow effective transduction of generated signals. The related device was capable of detecting light ranging from UV to NIR range (400-1575 nm). At room temperature, due to thermally activated band-to-band population of charge carriers in this active material, the $\mathrm{Fe}_{3}(\mathrm{HTTP})_{2}$ possessing a narrow IR band gap of $\sim 0.45 \mathrm{eV}$ displayed limited performance. Cooling the devices to $77 \mathrm{~K}$ suppressed this thermal excitation hence leading to a stronger response and higher sensitivity (Fig. 23b-d). Upon $785 \mathrm{~nm}$ excitation at $77 \mathrm{~K}$, a lower noise equivalent power was achieved with a specific detectivity of $7 \times 10^{8} \mathrm{~cm} \mathrm{~Hz}^{1 / 2} \mathrm{~W}^{-1}$.

\subsection{Spintronics}

Merging high conductivity and long range magnetic ordering are highly desirable in spintronics for data storage and processing, such as spin transistors, ${ }^{180}$ spin Hall effect transistors, ${ }^{181}$ spin photovoltaic, ${ }^{182}$ spin valves, ${ }^{183}$ etc. The traditional active materials such as inorganic compounds or organic/molecular film semiconductors have displayed high performance. Nevertheless, the inorganic compounds and organic/molecular film semiconductors are facing the challenge of lack of tunability in magnetic/conductive properties or adequacy in sufficient mobilities, respectively. ${ }^{32}$ By contrast, in-plane fully $\pi$-conjugated $2 \mathrm{D}$ $c$-MOFs with structural diversity and high charge transport enable the interaction between the carriers and localized spins, hence providing promise of tunable magnetic ordering. ${ }^{32,67,184}$

6.3.1 Ferromagnetic semiconductors based on 2D c-MOFs. Ferromagnetic (FM) semiconductors as emerging materials have attracted considerable attention as FM electrodes in the next generation logic and memory devices. ${ }^{151,183,185-189}$ In 2018 , our group reported the FM properties of the 2D $c$-MOF. ${ }^{32} \mathrm{~A}$ FM semiconductor based on the non-porous coronene-based
$\mathrm{Fe}_{3}$ (PTC) 2D c-MOF (Fig. 24a) presented a FM ground state at $\sim 15 \mathrm{~K}$ in the magnetic susceptibility measurements, which is in accordance with the exchange energy $\left(E_{\text {exchange }}=E_{\text {anti-FM }}-\right.$ $E_{\mathrm{FM}}$ ) of $1.22 \mathrm{meV}$ and an estimated Curie point of $\sim 16 \mathrm{~K}$ by DFT simulation (Fig. 24b and c). The distances of neighboring Fe atoms in $\mathrm{Fe}_{3}$ (PTC) were estimated as 0.54-1.20 and $\sim 0.5 \mathrm{~nm}$ in the in-plane and out-of-plane direction respectively, which excludes the strong exchange between the $\mathrm{d}$ orbitals from $\mathrm{Fe}$ atoms directly. Therefore, we proposed an indirect exchange through the network for the observed ferromagnetism and highlighted the impact of high $\pi-\mathrm{d}$ hybridization between the $\mathrm{d} / \mathrm{p}$ orbitals of the $\mathrm{FeS}_{4}$ complex and coronene on the indirect exchange interaction. Later on, we developed another FM semiconductor based on the $\mathrm{Fe}_{2}\left[\mathrm{FePcO}_{8}\right]$ 2D $c$-MOF (Fig. 24d). ${ }^{33}$ DFT calculations revealed a higher exchange energy of $\sim 300 \mathrm{meV}$ for FM coupling and suggested again an indirect exchange interaction benefiting from the strong $\pi / d$ hybridization of the orbitals between $\mathrm{FeO}_{4}$ and iron phthalocyanine. SQUID magnetometry showed a blocked superparamagnetic nature in $\mathrm{Fe}_{2}\left[\mathrm{FePcO}_{8}\right]$ due to the limited crystal size in the achieved sample and maintained magnetic ordering even at $350 \mathrm{~K}$ (Fig. 24e and f).

The contribution of strong $\pi-\mathrm{d}$ hybridization and strong $\pi$-conjugation to the charge transport along the entire network remains an open question. Nevertheless, we noted their influence on the extraordinary magnetic ordering among 2D $c$-MOFs. We also consider that both studies utilizing bulk materials did not exclude the impact of the grain boundaries, defects, edges, or the crystallite tilting on the magnetic properties. Improving the crystallinity to achieve single-crystals or single domain films will benefit the understanding of intrinsic magnetic properties and realizing ferromagnetism even at/ above room temperature.

6.3.2 Nonmagnetic 2D c-MOFs as spacers. In addition to the compelling FM properties, nonmagnetic 2D $c$-MOFs are able to function as channel materials for spacers in spin valves or spin transistors. ${ }^{183}$ In 2019, Song et al. applied the $\mathrm{Cu}_{3}(\mathrm{HHTP})_{2}$ film to fabricate organic spin valves (OSVs) with vertical architecture (Fig. 24g) and studied its spin injection and transport properties. ${ }^{97}$ The authors selected initially the $100 \mathrm{~nm}$ thick $\mathrm{Cu}_{3}(\mathrm{HHTP})_{2}$ as the channel material between the top (Co) and bottom $\left(\mathrm{La}_{0.67} \mathrm{Sr}_{0.33} \mathrm{MnO}_{3}, \mathrm{LMSO}\right) \mathrm{FM}$ electrodes to minimize the penetration of Co into the 2D $c$-MOF network in this device configuration and prevent possible short circuit. The fabricated OSV exhibited a large negative magnetoresistance (MR) of $25 \%$ at $10 \mathrm{~K}$ (Fig. $24 \mathrm{~h}$ ), and this negative MR could still be observed at temperature up to $200 \mathrm{~K}$.

However, the porous structure of $\mathrm{Cu}_{3}(\mathrm{HHTP})_{2}$ as a spacer in a vertical spin device may affect its long-term practicability due to the potential inter-diffusion of the upper electrode material of Co through the $1 \mathrm{D}$ channel of $\mathrm{Cu}_{3}(\mathrm{HHTP})_{2}$ to the LMSO electrode at the bottom. ${ }^{190}$ Therefore, non-porous networks for preventing the penetration are more reasonable. Instead, the lateral architecture represents another approach for the integration of porous $2 \mathrm{D} c$-MOFs into spintronic devices (Fig. 24i). 
a
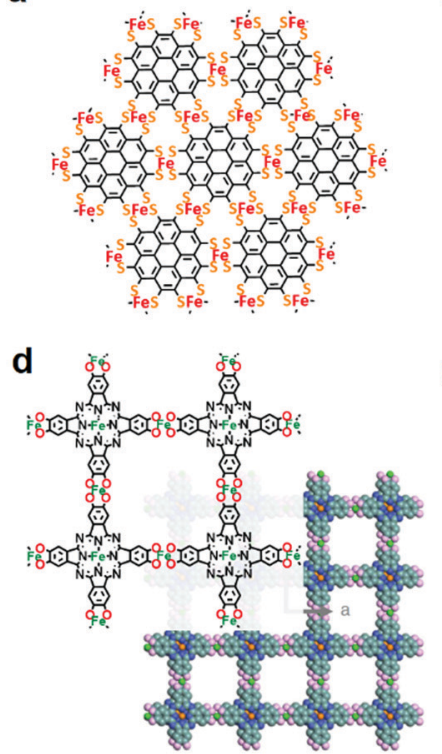

g

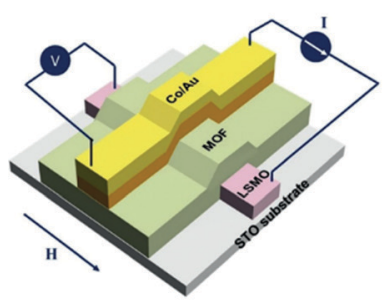

b

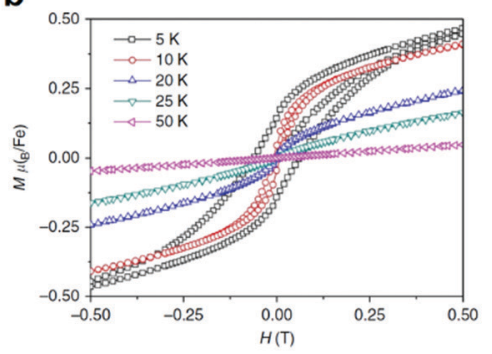

e

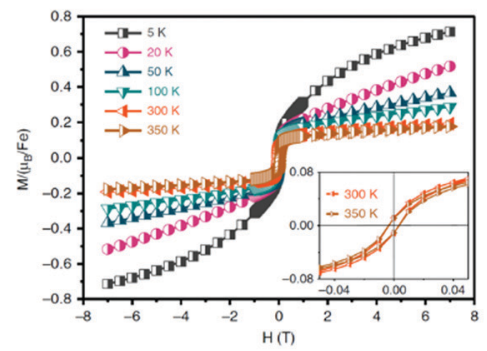

h

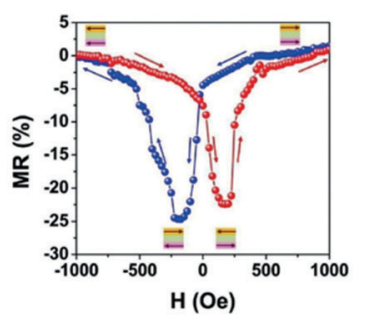

C

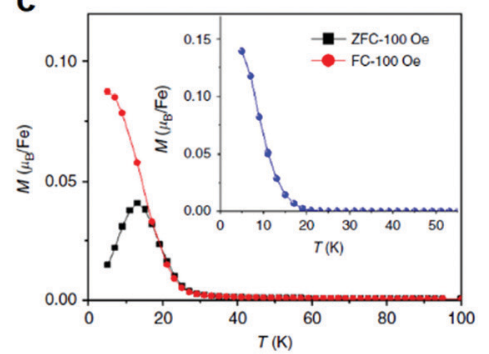

f

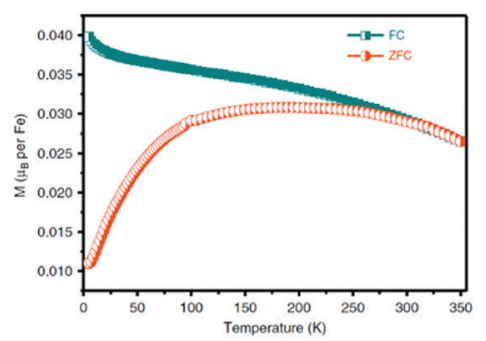

i

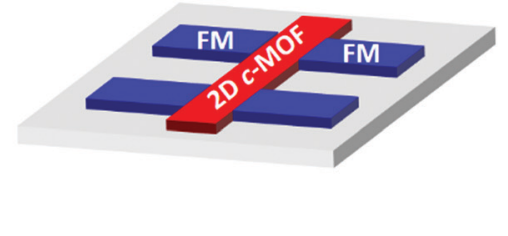

Fig. 24 Spin-properties of 2D c-MOFs. (a-c) $\mathrm{Fe}_{3}(\mathrm{PTC}) 2 \mathrm{D} \mathrm{c}$-MOF and its ferromagnetic ground state, magnetic hysteresis loops at different temperatures, as well as field-cooled (FC)/zero-field-cooled (ZFC) magnetization as a function of temperature at an applied field of 100 Oe. ${ }^{32}$ Copyright 2018, Nature Publishing Group. (d-f) $\mathrm{Fe}_{2}\left[\mathrm{FePcO}_{8}\right] 2 \mathrm{D} \mathrm{c}-\mathrm{MOF}$ and its magnetic hysteresis loops at different temperatures, ZFC/FC magnetization at 100 Oe. ${ }^{33}$ Copyright 2019, Nature Publishing Group. (g and h) Diagram of the $\mathrm{Cu}_{3}(\mathrm{HHTP})_{2}$ based vertical OSV and its magnetoresistance loop for at $10 \mathrm{~K}$. Reproduced with permission ref. 97. Copyright 2020, Wiley-VCH. (i) Schematic illustration of lateral spin valves.

\subsection{Thermoelectrics}

The porous structure of 2D $c$-MOFs makes them great candidate for thermoelectric applications because the pores are capable of scattering phonons strongly. ${ }^{105,110}$ In 2017, Sun et al. applied $\mathrm{Ni}_{3}(\mathrm{HITP})_{2}$ to the investigation of its thermoelectric properties though a pellet sample. ${ }^{105}$ The device of the pellet showed an ultralow thermal conductivity of $0.21 \mathrm{~W} \mathrm{~m}^{-1} \mathrm{~K}^{-1}$ and a high thermoelectric figure of merit $(Z T)$ of $1.19 \times 10^{-3}$ at $25{ }^{\circ} \mathrm{C}$. The $Z T$ value increased 17 -fold compared with traditional 7,7,8,8-tetracyanoquinodimethane-infiltrated $\mathrm{Cu}_{3}$ (benzene-1,3,5-tricarboxylate $)_{2} \quad\left(\mathrm{Cu}_{3}\right.$ (BTC) ${ }_{2}$-TCNQ) MOF based thermoelectric device. The room temperature conductivity of $58.8 \mathrm{~S} \mathrm{~cm}^{-1}$ correlates to an electronic $\left(\kappa_{\mathrm{e}}\right)$ contributed thermal conductivity of $4.28 \times 10^{-3} \mathrm{~W} \mathrm{~m}^{-1} \mathrm{~K}^{-1}$, hence suggesting a lattice dominated thermal transport in this $2 \mathrm{D} c$-MOF.

$\mathrm{Up}$ to here, despite the great progress of $2 \mathrm{D} c$-MOFs for (opto)electronics, spintronics, and thermoelectrics, the current studies in these fields rely largely on TP-based 2D $c$-MOFs, due to the contributions of diverse synthetic methodologies for the TP-based systems. The development of MOFtronics towards other 2D $c$-MOF systems is still in its infancy. In-depth understanding on producing/integrating methods and exploring active materials with appropriate conductivity and band structure merits further exploration.

\subsection{Electrochemical energy storage devices}

Conductive 2D $c$-MOFs incorporating triphenylene-type monomers and abundant quinone-species, such as $o$-benzosemiquinonate and $o$-diiminobenzosemiquinonate (Fig. 7), ${ }^{36,40,51,56,57,191}$ have emerged as a novel class of multifunctional electrode materials for electrochemical energy storage. Among plenty of technologies, ${ }^{104,192-196}$ metal-ion batteries and supercapacitors represent two major energy storage devices. ${ }^{195}$ Benefiting from the intrinsically conductive and porous properties, 2D $c$-MOFs have shown excellent performance in metal-ion batteries and supercapacitors, which could function as the power sources for the above mentioned MOFtronics. ${ }^{197-201}$

6.5.1 Metal-ion batteries. Benzene-based 2D $c$-MOFs with dense redox active sites have been widely utilized as active cathode materials for lithium/sodium-ion batteries (Fig. 25a). ${ }^{35,36,87,88,202}$ As a typical example, Wada et al. reported the utilization of $\mathrm{Ni}_{3}(\mathrm{HIB})_{2}$ 2D $c$-MOFs in Li-ion batteries in $2018 .^{36}$ As a cathode material, $\mathrm{Ni}_{3}(\mathrm{HIB})_{2}$ delivered a high specific capacity of $155 \mathrm{~mA} \mathrm{~h} \mathrm{~g}{ }^{-1}$ in a $1 \mathrm{M} \mathrm{LiPF}_{6}$ electrolyte (Fig. 25b). Based on XPS and DFT, the authors proposed a 
charge re-arrangement between $\mathrm{N}$ and $\mathrm{Ni}$ atoms once electron was injected, which shifts $\mathrm{Ni}_{3}(\mathrm{HIB})_{2}$ to a more stable structure. The bottom-up synthesized $\mathrm{Cu}_{3}(\mathrm{HHB})_{2}$ nanosheets (Section 5) hold promise for lithium-ion batteries owing to the high exposure of active sites. ${ }^{8}$ The discharge capacity was determined as $153 \mathrm{~mA} \mathrm{~h} \mathrm{~g}^{-1}$ for the nanosheet samples in $1 \mathrm{M} \mathrm{LiPF}_{6}$ at $0.1 \mathrm{~A} \mathrm{~g}^{-1}$ in $1.3-2.6 \mathrm{~V} \mathrm{vs.} \mathrm{Li}^{+} / \mathrm{Li}$, and it was further pushed to $400 \mathrm{~mA} \mathrm{~h} \mathrm{~g}{ }^{-1}$ in the potential window of 1.3-4.0 V (Fig. 25c). The prepared battery exhibited outstanding cycling stability with a capacity retention of $90 \%$ after 1000 cycles. The ultrathin feature from the nanosheets for fast ion diffusion and high utilization of active sites was highlighted, while bulk $\mathrm{Cu}_{3}(\mathrm{HHB})_{2}$ powder provided limited performance. Park et al. utilized $\mathrm{Co}_{3}(\mathrm{HIB})_{2}$ for Na-ion storage in 2018. ${ }^{35}$ This $2 \mathrm{D} c$-MOF was found to be capable of storing three $\mathrm{Na}^{+}$per HIB ligand (corresponding to two $\mathrm{Na}^{+}$per $\mathrm{CoN}_{4}$ linkage) with $1 \mathrm{M}$ $\mathrm{NaPF}_{6}$ electrolyte. As a result, an ultrahigh specific capacity of $291 \mathrm{~mA} \mathrm{~h} \mathrm{g^{-1 }}$ was recorded. XPS analysis suggested that the reversible redox reactions mainly proceeded on the $o$-diiminobenzosemiquinonate moiety, since no obvious valence change for $\mathrm{Co}^{2+}$ was detected. $\mathrm{Ni}_{3}(\mathrm{HIB})_{2}$ exhibited a similar capacity of $295 \mathrm{~mA} \mathrm{~h} \mathrm{~g}{ }^{-1}$ under identical conditions.

Although Li/Na-ion batteries have been extensively studied, the high cost and safety issues impede further development. Instead, less toxic and cheaper aqueous zinc batteries are a good alternative. Recently, Nam et. al. developed a $\mathrm{Cu}_{3}(\mathrm{HHTP})_{2}$ based $\mathrm{Zn}$-ion battery (Fig. 25d) with a high capacity of $228 \mathrm{~mA} \mathrm{~h} \mathrm{~g}^{-1}$. $^{89}$ The authors identified the role of $\mathrm{H}_{2} \mathrm{O}$ in the performance. In an aqueous electrolyte of a $3 \mathrm{M}$ aqueous solution of $\mathrm{Zn}\left(\mathrm{CF}_{3} \mathrm{SO}_{3}\right)_{2}$, the hydrated $\mathrm{Zn}^{2+}$ was allowed to be

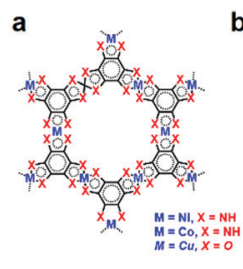

d

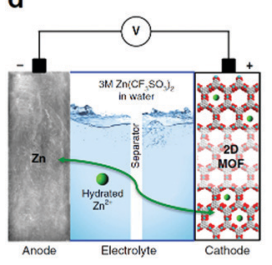

b
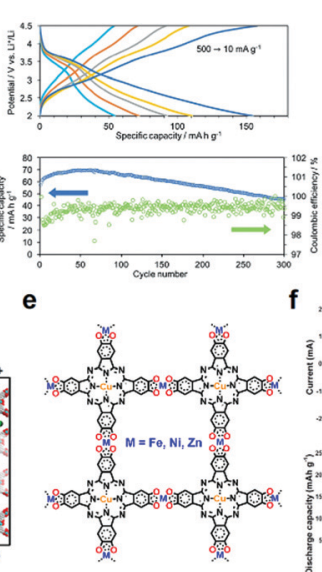

C

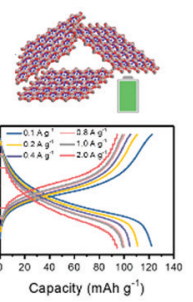

f

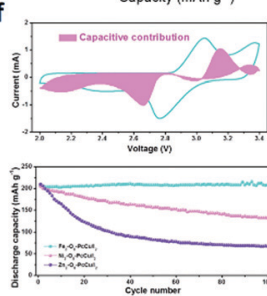

Fig. 25 Selected 2D C-MOF based metal-ion batteries. (a) Applied $\mathrm{M}_{3}(\mathrm{HXB})_{2} 2 \mathrm{D}$ c-MOFs for metal-ion batteries. When $M=\mathrm{Cu}, \mathrm{X}=\mathrm{O}$, the charge state is -1 . (b) The charge/discharge curves and cycling stability test of $\mathrm{Ni}_{3}(\mathrm{HIB})_{2}$ in a Li-ion battery. Reproduced with permission ref. 36 . Copyright 2018, Wiley-VCH. (c) Utilization of $\mathrm{Cu}_{3}(\mathrm{HHB})_{2}$ thin nanosheets in $\mathrm{Li}$-ion batteries and the charge/discharge curves. ${ }^{88}$ Copyright 2020, the Royal Society of Chemistry. (d) Illustration of $\mathrm{Cu}_{3}(\mathrm{HHTP})_{2}$ based aqueous Zn-ion battery. ${ }^{89}$ Copyright 2019, Nature Publishing Group. (e and f) $\mathrm{M}_{2}\left[\mathrm{CuPCO}_{8}\right]$ as the host in a $\mathrm{Na}-\mathrm{I}_{2}$ battery and its electrochemical performance, the calculated capacitive contribution of the $\mathrm{Fe}_{2}\left[\mathrm{CuPcO}_{8}\right] /$ $\mathrm{I}_{2}$ electrode as well as the cycling stability. ${ }^{203}$ Copyright 2020 , Wiley-VCH. inserted into the 1D channels in the backbone with a high diffusion rate during discharge. As contrast, significantly higher interfacial resistance and a lower capacity of $144 \mathrm{~mA} \mathrm{~h} \mathrm{~g}^{-1}$ were recorded for the organic electrolyte.

Apart from serving as active materials, our group has explored 2D $c$-MOFs as the host for the non-conductive cathode material of iodine $\left(\mathrm{I}_{2}\right)$ in Na- $\mathrm{I}_{2}$ batteries. ${ }^{203} \mathrm{M}_{2}\left[\mathrm{CuPcO}_{8}\right](\mathrm{M}=\mathrm{Fe}$, $\mathrm{Ni}, \mathrm{Zn}$ ) were selected for hosting iodine and resolving the poor cycle stability of the $I_{2}$ cathode due to the dissolution of polyiodide in the electrolyte. The $\mathrm{MO}_{4}$ linkages were confirmed to be effective for iodine adsorption; amongst, $\mathrm{FeO}_{4}$ possessed the highest efficacy. The constructed $\mathrm{Fe}_{2}\left[\mathrm{CuPcO}_{8}\right] / \mathrm{I}_{2}$ electrode delivered a high discharge capacity of $208 \mathrm{~mA} \mathrm{~h} \mathrm{~g}{ }^{-1}$ and outstanding cycling stability with almost no capacity decay after 3200 cycles (Fig. 25e and f).

6.5.2 Supercapacitors. 2D $c$-MOFs have also represented promising electroactive materials for electrical double layer (EDL) capacitors, ${ }^{37,101,146,199}$ which possess a fast charge/ discharge process, excellent cyclability, and superior power density with respect to metal-ion batteries. ${ }^{204}$ The capacitance of EDL capacitors arises from physical ion adsorption and charge separation on the electrode surface. In 2016, Sheberla et al. reported the exploration of $\mathrm{Ni}_{3}(\mathrm{HITP})_{2}$ for $\mathrm{EDL}$ capacitors. ${ }^{37}$ Pelletized $\mathrm{Ni}_{3}(\mathrm{HITP})_{2}\left(\sigma=50 \mathrm{~S} \mathrm{~cm}^{-1}\right)$ with unique 1D channels with diameters of $\sim 1.5 \mathrm{~nm}$ exhibited ideal EDL charge storage with a high gravimetric capacitance of $\sim 110 \mathrm{~F} \mathrm{~g}^{-1}$ and an areal capacitance of $\sim 18 \mu \mathrm{F} \mathrm{cm} \mathrm{cm}^{-2}$ at a discharge rate of $0.05 \mathrm{~A} \mathrm{~g}^{-1}$. The authors highlighted the role of the $1 \mathrm{D}$ channel in the diffusion and accommodation of the electrolyte ions to the EDL storage (Fig. 26a). To access insights into the relationship between the pore diameters and the performance, Bi et al. performed constant-potential molecular dynamic simulations on three types of 2D $c$-MOFs with room-temperature ionic liquids (RTILs) as the electrolyte. ${ }^{205}$ The $2 \mathrm{D} c$-MOFs, $\mathrm{Ni}_{3}(\mathrm{HHB})_{2}, \mathrm{Ni}_{3}(\mathrm{HITP})_{2}$, and $\mathrm{Ni}_{3}(\mathrm{HITN})_{2}(\mathrm{HITN}=$ 2,3,8,9,14,15-hexaiminotrinaphthylene), feature different pore sizes in the range of 0.8 to $2.4 \mathrm{~nm}$ (Fig. 26b). Among them, $\mathrm{Ni}_{3}(\mathrm{HITN})_{2}$ with the largest pore was estimated to exhibit the highest gravimetric capacitance and energy density. However, theory inferred highest values with respect to volumetric capacitance and energy density for $\mathrm{Ni}_{3}(\mathrm{HHB})_{2}$ with the narrowest $1 \mathrm{D}$ channel. On further reducing the pore size, ions could barely enter the channels leading to smaller or zero capacitance. In addition, the less conductive $\mathrm{Cu}_{3}(\mathrm{HHTP})_{2}$ was assembled directly onto a carbon fiber paper (as current collector) to function as electrodes in a EDL capacitor, ${ }^{90}$ which exhibited a gravimetric capacitance of $120 \mathrm{~F} \mathrm{~g}^{-1}$ and an areal capacitance of $\sim 22 \mathrm{mF} \mathrm{cm}^{-2}$ at $0.5 \mathrm{~A} \mathrm{~g}^{-1}$ in polyvinyl alcohol (PVA)/KCl gel electrolyte (Fig. 26c). The authors highlighted the mechanical adhesion between the current collector and the assembled 2D cMOF on top for effective charge transport. Unsurprisingly, the electrode composed of coated bulk $\mathrm{Cu}_{3}(\mathrm{HHTP})_{2}$ with a polymer binder on the carbon paper is not that effective.

Apart from the conventional supercapacitors, ${ }^{206,207}$ efforts have been devoted to flexible micro-supercapacitors (MSCs) as well, which are promising micro-power sources for next-generation 


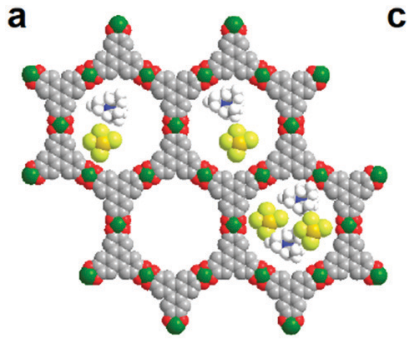

C

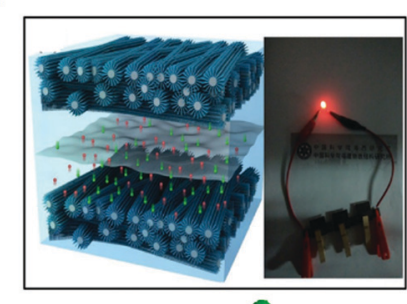

b

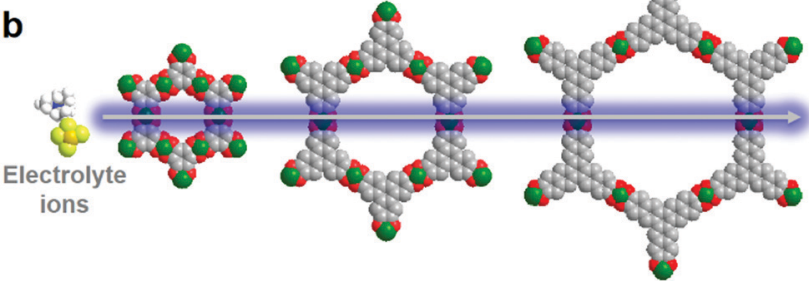

Fig. 26 EDL capacitors based on 2D c-MOFs. (a) Schematic of the accommodation of electrolyte ions in 2D c-MOF. (b) Schematic of the diffusion of electrolyte ions into various 2D c-MOFs with different sized 1D channels. (c) $\mathrm{Cu}_{3}(\mathrm{HHTP})_{2}$ on carbon fiber paper for supercapacitors, which powered a red light-emitting-diode. Reproduced with permission ref. 90 . Copyright 2017, Wiley-VCH.

portable electronic devices. ${ }^{208-211}$ Very recently, Wu et al. applied laser scribing technique assisted solvothermal synthesis to interdigitate $\mathrm{Ni}_{9}(\mathrm{HHTP})_{4}$ on 3D porous laser scribed graphene (LSG) on a polyimide (PI) substrate. ${ }^{100} \mathrm{~A} \mathrm{CO}_{2}$ laser was applied for patterning LSG, followed by $\mathrm{O}_{2}$-plasma treatment to serve as a current collector and the platform for the deposition of $\mathrm{Ni}_{9}(\mathrm{HHTP})_{4}$ (Fig. 27a and b). The hybrid delivered areal capacitance, energy density, and power density of $15.2 \mathrm{mF} \mathrm{cm}^{-2}, 4.1 \mu \mathrm{W} \mathrm{h} \mathrm{cm}{ }^{-2}$, and $7 \mathrm{~mW} \mathrm{~cm}^{-2}$, respectively. Benefiting from the ultrathin feature of our exfoliated $\mathrm{Ni}_{2}\left[\mathrm{CuPc}(\mathrm{NH})_{8}\right]$ nanosheets (Section 5) that allow facile solutionprocessibility through filtration, flexible MSC devices were constructed based on $\mathrm{Ni}_{2}\left[\mathrm{CuPc}(\mathrm{NH})_{8}\right]$ /graphene hybrids on PI as film electrodes in a PVA/LiCl gel electrolyte (Fig. 27c). ${ }^{55}$ The MSC device exhibited high areal capacitance and power density

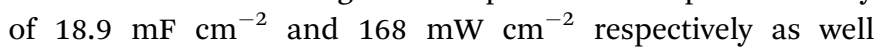
as excellent cycling stability. Even on alternating the flat and bent states, the device still maintained $86.2 \%$ of the initial performance after 3000 cycles (Fig. 27d).

Beside EDL storage, 2D $c$-MOFs have displayed pseudocapacitive charge storage $e^{25,26}$ in aqueous electrolytes, which differs from the EDL mechanism and involves surface reversible faradaic redox reactions. ${ }^{206}$ The incorporation of the pseudo-capacitive storage with redox reactions generally leads to enhancement in gravimetric and volumetric capacitances. In 2018, Feng et al. reported pseudo-capacitors based on $\mathrm{Cu}_{3}(\mathrm{HIB})_{2}$ and $\mathrm{Ni}_{3}(\mathrm{HIB})_{2}$ in $1 \mathrm{M} \mathrm{KOH}$ aqueous electrolyte. ${ }^{38}$ The reversible pseudo-capacitive behaviors enabled $\mathrm{Cu}_{3}(\mathrm{HIB})_{2}$ and $\mathrm{Ni}_{3}(\mathrm{HIB})_{2}$ pellets to present a high gravimetric capacitance of 215 and over $400 \mathrm{~F} \mathrm{~g}^{-1}$, respectively, while the contribution of EDL capacitances was estimated to be less than $20 \%$ and $10 \%$, respectively. A $50 \mu \mathrm{m}$ thick $\mathrm{Ni}_{3}(\mathrm{HIB})_{2}$ exhibited an excellent volumetric capacitance up to $760 \mathrm{~F} \mathrm{~cm}^{-3}$. By further a

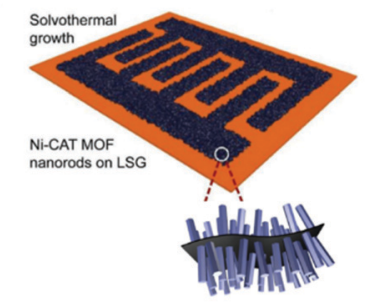

C

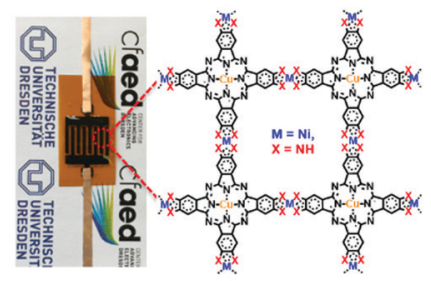

b

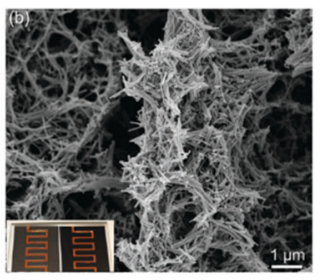

d

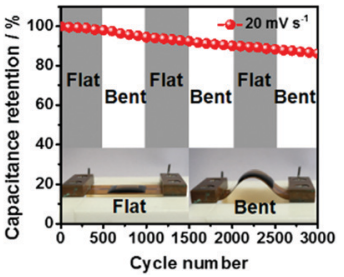

Fig. 27 2D c-MOFs based MSCs. (a and b) Illustration of LSG-supported $\mathrm{Ni}_{9}(\mathrm{HHTP})_{4}$ for MSC and its SEM image. Reproduced with permission ref. 100. Copyright 2019, Wiley-VCH. (c and d) Flexible MSC device based on $\mathrm{Ni}_{2}\left[\mathrm{CuPC}(\mathrm{NH})_{8}\right]$ nanosheets. ${ }^{55}$ Copyright 2020, Wiley-VCH.

thickening it to $360 \mu \mathrm{m}$, the volumetric capacitance was revealed to be $460 \mathrm{~F} \mathrm{~cm}^{-3}$ and an areal capacitance was determined to be $17 \mathrm{~F} \mathrm{~cm}^{-2}$. In addition, similar to those metal-ion batteries, ${ }^{35,36}$ the redox reaction in $\mathrm{Ni}_{3}(\mathrm{HIB})_{2}$ was speculated to be ligand-based, ${ }^{37}$ as evidenced by a set of redox peaks from the ligand and excluded valence change of $\mathrm{Ni}$ atoms by XPS. Pseudo-capacitive behavior was further exemplified by the DBC-based $\mathrm{Cu}_{2}\left[\mathrm{DBCO}_{8}\right] 2 \mathrm{D} c$-MOF. ${ }^{56}$ In a $1 \mathrm{M} \mathrm{NaCl}$ aqueous electrolyte, $\mathrm{Cu}_{2}\left[\mathrm{DBCO}_{8}\right]$ displayed a nearly rectangular cyclic voltammetry curve with distinct redox peaks, indicative of both EDL and pseudo-capacitance for this material with estimated contributions of 62 and $38 \%$, respectively. The supercapacitor provided high areal and volumetric capacitances of $\sim 0.9 \mathrm{~F} \mathrm{~cm}^{-2}$ and $22 \mathrm{~F} \mathrm{~cm}^{-3}$ respectively in solid-state.

As demonstrated, owing to the high intrinsic porosity and abundant redox active linkages, 2D $c$-MOFs have been integrated into multifunctional electrochemical energy storage devices. Hybridization ${ }^{212-216}$ of 2D $c$-MOFs with other materials has been proved to be an approach for advancing the performance. Accordingly, various templates, such as carbon fiber paper,${ }^{90}$ cellulose nanofibers ${ }^{101}$ graphene, ${ }^{100}$ copper metal, ${ }^{217}$ and polypyrrole membrane ${ }^{146}$ have been utilized as platforms for the deposition of $2 \mathrm{D} c$-MOFs. However, these samples are not structurally defined 2D $c$-MOFs anymore and exclude a neat structure-property relationship. Overall, the current studies provide guidelines corresponding to the rational design of chemical structure and modulation on the porosity and pore size.

\section{Conclusions and outlook}

In this review article, we introduce the diversity of the chemical structures of 2D $c$-MOFs based on various conjugated building blocks including benzene, triphenylene, trinaphthylene, truxene, hexaazatrinaphthylene, coronene, phthalocyanine, 
naphthalocyanine, and dibenzo $[g, p]$ chrysene with orthosubstituted functional groups $\left(-\mathrm{OH},-\mathrm{NH}_{2},-\mathrm{SH}\right.$, and $\left.-\mathrm{SeH}\right)$. In addition, the multicomponent $2 \mathrm{D} c$-MOFs comprising mixed ligands (e.g. $\mathrm{Cu}_{3}(\mathrm{HHTP})(\mathrm{HHB})$ ) or mixed metals (e.g. $\mathbf{M}_{n} \mathbf{M}_{3-n}^{\prime}(\mathrm{HITP})_{2}$ ) show great potential of tunability of the porosity, active sites, and electronic properties. The chemical tunability endows 2D $c$-MOFs with unique chemical and physical features, such as crystallinity, porosity, stability, tailorable band gaps, electrical conductivity, photoactivity, magnetic ordering, and topological state, thereby offering 2D $c$-MOFs great potential for broad applications in multiple MOFtronics. In this context, based on the thus-far reported examples, we provide an overview of the monomer/2D $c$-MOF design, controlled synthesis, device integration, and multifunction of 2D $c$-MOFs.

More specifically, we discuss the structure-electronic property relationship by considering the influence of molecular design (type and geometry of ligand, metal) and material structure (redox-activity in linkage, layer stacking and arrangement) as well as crystallinity. We further summarize the conductivities and mobilities for thus-far reported 2D $c$-MOFs, which reach $2500 \mathrm{~S} \mathrm{~cm}^{-1}\left(\mathrm{Cu}_{3}(\mathrm{HTB})\right.$, measured by 4-probe configuration) and $220 \mathrm{~cm}^{2} \mathrm{~V}^{-1} \mathrm{~s}^{-1}\left(\mathrm{Fe}_{3}(\mathrm{HTTP})_{2}\right.$, hole mobility measured by Hall effect and TRTS) respectively at room temperature. Theoretically, DFT calculations demonstrated that 2D $c$-MOFs are expected to exhibit some nontrivial physical properties, such as topological state (single-layer of $\mathrm{Ni}_{3}(\mathrm{HTB})_{2}$ ), high-temperature ferromagnetic ordering (Curie temperature of 170,212 , and $450 \mathrm{~K}$ for single-layer of $\mathrm{Ni}_{2}\left[\mathrm{MnPc}\left(\mathrm{NH}_{8}\right)\right]$, $\mathrm{Mn}_{3}(\mathrm{HTB})_{2}$, and $\mathrm{Mn}_{3}(\mathrm{HIB})_{2}$, respectively). ${ }^{34,84,85}$ Experimentally, various synthetic methodologies have been applied to the development of 2D c-MOFs as bulk powder (hydro-/solvothermal method) or film (wet-interface-assisted method) samples. The hydro-/solvothermal method is a straightforward strategy that allows diversity in the reaction conditions and offers large scale and high yield synthesis. Besides the generally obtained morphology of 2D $c$-MOFs as nanocrystals $<100 \mathrm{~nm}$, this method also provides the possibility to synthesize moderate-sized single-crystals $(1-10 \mu \mathrm{m})$. Among the reported systems, we highlight the significance of single-crystalline nanorods (e.g. $\mathrm{Cu}_{3}(\mathrm{HHTP})_{2}, \mathrm{Ni}_{3}(\mathrm{HITP})_{2}$ ) and nanoflakes (e.g. $\left.\mathrm{Cu}_{3}(\mathrm{HHTP})_{2}, \mathrm{Cu}_{3}(\mathrm{HHB})_{2}\right)$ on unambiguous understanding of the charge transport properties/mechanisms of $2 \mathrm{D} c$-MOFs. By comparison, the wet-interface-assisted strategies including gas-liquid, liquid-liquid, and liquid-solid interfacial approaches show promise in constructing large-area, free-standing 2D $c$-MOF thin films, which ensure sufficient charge transport and facile device integration and hold potential for the realization of practical application of this class of materials for MOFtronics. Particularly, we highlight the construction of single-layer of 2D $c$-MOFs (e.g. $\mathrm{Ni}_{3}(\mathrm{HTTP})_{2}$, at the LB air/water interface), which is beneficial to the high exposure of active sites and excellent processibility. Next, device fabrications based on the obtained bulk or film samples are presented. To address the processing challenges faced by the bulk crystals, top-down exfoliation and bottom-up surfactant-assisted synthesis achieving homogeneous nanosheets in solution are considered as efficient strategies to provide accessible active sites and solution-processibility.

Finally, we introduce the broad application of conductive 2D $c$-MOFs for MOFtronics covering (opto)electronics (e.g. FETs, superconductors, chemiresistors, photodetectors), spintronics (e.g. ferromagnetic semiconductors, spacers), and thermoelectrics, as well as the utilization of 2D $c$-MOFs as potential power sources for MOFtronics including battery and supercapacitor devices. Owing to the unique features of 2D $c$-MOFs combining large surface to volume ratio, defined active sites, and tailored electronic structure with high conductivity, photoactivity, or high-temperature ferromagnetic ordering, we especially emphasize the great potential of 2D $c$-MOFs for superconductors, photodetectors, and ferromagnetic semiconductors.

In addition to the foregoing applications, 2D $c$-MOFs are also potential alternatives to traditional electrocatalysts for promoting the reactivity and fundamentally understanding the mechanism ${ }^{218-221}$ in various electrochemical energy conversion reactions. $^{222-229} \mathrm{MO}_{4^{-}}$and $\mathrm{MS}_{4}$-linkages have been widely demonstrated as the active sites towards the hydrogen evolution reaction ${ }^{45,49,61,62,118,230,231}$ and oxygen evolution reaction. ${ }^{54,160,232,233}$ For instance, the constructed single-layer of $\mathrm{Ni}_{3}(\mathrm{HTTP})_{2}$ exhibited an overpotential of $333 \mathrm{mV}{ }^{45}$ Apart from water splitting, 2D $c$-MOFs have emerged as model catalysts to investigate the mechanism during the oxygen reduction reaction. ${ }^{52,99,103,234-238}$ For instance, $\mathrm{Co}_{2}\left[\mathrm{CuPcO}_{8}\right]$ delivered excellent activity with a half-wave overpotential of $830 \mathrm{mV}$, under a four-electron $\mathrm{O}_{2}$ reduction process. ${ }^{53}$ In regard to the emerging carbon dioxide reduction reaction $\left(\mathrm{CO}_{2} \mathrm{RR}\right)$, based on an array of $\mathrm{M}_{2}\left[\mathrm{M}^{\prime} \mathrm{PcO}_{8}\right]\left(\mathrm{M}=\mathrm{Cu}, \mathrm{Zn} ; \mathrm{M}^{\prime}=\mathrm{Cu}, \mathrm{Zn}\right) 2 \mathrm{D} c$ MOFs with finely tuned active sites, our group has established a structure-property relationship regarding the selectivity of active sites toward carbon dioxide reduction or the concomitant hydrogen evolution. ${ }^{53}$ Regardless of the preliminary success, the study towards complicated reactions such as $\mathrm{CO}_{2} \mathrm{RR}$ is still at infancy, and in-depth investigation on the mechanisms remains of growing interest. In the past few years, 2D $c$-MOFs stand out for electrochemical molecular capture ${ }^{109}$ and detection $^{239,240}$ as well.

The development of 2D $c$-MOFs discussed here leaves no doubt that the extension of this class of materials to MOFtronics is of substantial interest to the next generation of logic and memory devices. In this context, it is important to clarify that the traditional photo-/electroactive materials should not be the metric for 2D $c$-MOFs and MOFtronics. 2D $c$-MOFs may outperform traditional electronic materials, but, this judgement cannot be true without specifying the particular property and device/technique type. For instance, the intrinsic room-temperature mobilities of electrons and holes for crystalline silicon were recorded in the range of 1450-1610 and $300-400 \mathrm{~cm}^{2} \mathrm{~V}^{-1} \mathrm{~s}^{-1}$, respectively via the Hall effect, which is far beyond the thus-far reported highest values recorded by the same technique for $2 \mathrm{D} c$-MOFs $\left(220 \mathrm{~cm}^{2} \mathrm{~V}^{-1} \mathrm{~s}^{-1}\right)$. In spite of whether 2D $c$-MOFs become better than silicon in the future, MOFtronics will likely never replace the silicon-electronics. Besides, 2D c-MOFs represent layer-stacked framework materials 
and the development of single- or few-layer 2D $c$-MOFs is still in its infancy. The lack of control on layer number and the ambiguous contribution of in-plane charge transport lead to difficulties to compare the electronic properties of $2 \mathrm{D} c$-MOFs with other $2 \mathrm{D}$ materials. Nevertheless, the reported mobilities for 2D $c$-MOFs significantly exceed those of typical crystalline organic semiconductors (mostly $<20 \mathrm{~cm}^{2} \mathrm{~V}^{-1} \mathrm{~s}^{-1}$ ). The band gaps of 2D $c$-MOFs exhibit a range of values comparable to amorphous organic molecules/polymers. In addition, for semiconducting organic molecule/polymer single-crystals, it is quite complicated to thin the crystals into single- or few-layers, whereas free-standing 2D $c$-MOF nanosheets/films represent a kind of advantage in processing and device integration. Therefore, it is clear that 2D c-MOFs are a unique class of electronic materials that exhibit exceptional properties, i.e., highly tailorable band structure, high porosity and stability. The immense progress from synthesis to function are highly encouraging and validate their unique properties underlying the applications. Nevertheless, the practical utilization of $2 \mathrm{D}$ $c$-MOFs beyond laboratories is yet to be realized. Many challenges remain to be addressed for ascertaining a neat structure-property relationship and promoting the development of this field.

(i) As illustrated, the library of 2D $c$-MOFs have considerably expanded with the development of novel ligands and synthetic methodologies. The known layered 2D $c$-MOFs exhibit anisotropic transport features; the strong $\pi-\mathrm{d}$ hybridization and extended $\pi$-conjugation together with the interlayer $\pi-\pi$ interactions contribute to their charge transport. Nonetheless, the current ligand design and linkage chemistry are quite limited. Regardless of that multicomponent 2D $c$-MOFs allow further expansion of chemical structures and show promise in the tunability of band gaps in fine-scale, related investigation remains rare. Regarding the development of chemical methodologies, rational design of both conjugated ligands and metal nodes is responsible for the high electrical conductivity. More specifically, the molecular design (ligand type/geometry/ size, metal valence/type, and $\pi$-conjugation degree) and the material structure (redox-activity in linkage, layer stacking and arrangement) are considered to be the major influential factors on the overall high conductivity of $2 \mathrm{D} c$-MOFs but remain immature. Concerning the redox-activity in linkage, precise modulation of charge states via redox reactions leading to charge carrier (free radicals) injection plays an important role in the charge transport, which is however unexplored for many negatively charged 2D $c$-MOFs $\left(e . g\right.$. $\mathrm{Cu}_{3}(\mathrm{HHB})_{2}$ with a charge state of -1). Meanwhile, after the redox reactions, thorough structural characterization including compositional and crystal analysis of 2D $c$-MOFs thus excluding potential decomposition/ doping would be beneficial to ascertain a neat structure-conductivity relationship.

(ii) An important aspect with respect to the recorded charge transport properties (e.g. conductivity, metallicity, mobility, etc.) is the absence of the contributions of boundaries, defects, and edges in the polycrystalline samples having small domains, which results in an ambiguous charge transport mechanism. Regarding the extraordinary high-temperature ferromagnetic ordering, despite the highlighted significance of strong $\pi-\mathrm{d}$ hybridization and $\pi$-conjugation, current investigation did not exclude these foregoing extrinsic factors. Thus, in terms of the fundamental physical property studies, it is clear that their boundaries and effects need to be considered and understood at a deeper level based on reliable methods and devices (e.g. single-crystal device). Improving the quality of crystals (crystallinity and domain size) to achieve single-crystals or singledomain films, controlling their morphology, and tuning the orientation of the nanocrystals thus minimizing the extrinsic influence are of key significance before establishing structureproperty relationships.

(iii) Proof-of-concept functional investigation of conductive 2D $c$-MOFs have been successfully demonstrated in various MOFtronics. Yet the thus-far fabricated devices through the compressing or drop-casting method based on bulk 2D $c$-MOFs have brought difficulties in realizing the mass production of devices. Unsurprisingly, the reported performances are still far from satisfaction. Furthermore, the current functional studies rely largely on triphenylene-based systems; MOFtronics towards other 2D $c$-MOFs is still in its infancy. In-depth thinking on improving the quality and mechanical strength of $2 \mathrm{D} c$-MOF thin films, optimizing the producing/integrating methods leading to high smoothness and good contacts, and exploring active materials with appropriate conductivity and band gap/structure remain to be explored. Moreover, fundamental understanding of the mechanism behind properties thus ascertaining neat structure-property relationship requires investigation. In addition to MOFtronics, the conductive and porous 2D $c$-MOFs also show great promise for developing future energy storage devices. Despite the substantial progress, challenges of probing the accurate charge storage mechanism on redox behavior and ion/electron diffusion remain ahead. In addition, utilization of these bulk porous materials through mechanical compression or deposition with support of binders on conductive platforms correlates with drawbacks including sacrificed pore volume and poor contact hence minimizing the performance in general, which rely on further optimizing the processing methods to overcome.

(iv) Currently, the novel device and new functions for $2 \mathrm{D}$ $c$-MOFs are largely limited. In regard to the nontrivial organic topological insulators, a single-crystalline monolayer of the $2 \mathrm{D}$ $c$-MOF (e.g. $\mathrm{Ni}_{3}(\mathrm{HTB})_{2}$ ) with a large size is still missing. The monolayer of 2D $c$-MOFs offers also other advantages: tunability at the atomic/molecular level and fabrication of 2D $c-\mathrm{MOF} /$ 2D $c$-MOF or 2D $c$-MOF/2D materials (e.g. graphene, $\mathrm{MoS}_{2}$ ) van der Waals heterostructures. Particularly, van der Waals heterostructures provide the possibility toward atomic/molecularlevel structure design and multifunctional properties by selecting appropriate candidates but remained unexplored. Therefore, the major challenges for pushing MOFtronics still rely on the controlled chemical synthesis.

(v) Except for the foregoing points, we face many questions on the synthetic methodologies of 2D $c$-MOFs, which hinder in principle the advancement of $2 \mathrm{D} c$-MOFs and MOFtronics with respect to property analysis, device integration, and function. Hydro-/solvothermal syntheses represent the most 
representative strategy, but they barely allow control of the crystal growth and the obtained bulk samples are associated with difficulties in sample processing and device integration. Exfoliation of the bulk crystals into nanosheets has been recently explored, but the quality of the obtained nanosheets relying on the bulk samples remains unsatisfied. As mentioned, the development of large-area, free-standing 2D $c$-MOFs thin/thick films and monolayers synthesized via wet interfaceassisted strategies allow facile device integration, sufficient charge transport, and fast signal transduction, hence offering the possibility toward high performance and long-term stability of MOFtronics. Nevertheless, the interfacial synthetic methods are limited to a certain range of systems (mainly based on benzene or triphenylene building blocks) hence limiting the application of other 2D $c$-MOFs. Towards controlled synthesis of 2D $c$-MOF crystals, rational design of the ligand structure/ chemistry, favoring high/moderate solubility in certain solvents and high reversibility during the metal coordination as well as re-balancing the competition between in-plane coordination and out-of-plane van der Waals interactions shall be essential to enlarge the crystal sizes and reduce defects and grain boundaries. In addition, high mechanical strength, controlled orientation, as well as fine-tuned thickness and lateral size of the films are still synthetic challenges. Given all that, the synthesis of 2D $c$-MOFs as single-crystals or single-domain monolayers is urgently required for future advancement. ${ }^{241}$

Over eight years of development, despite many open questions and the huge gap between this stage and the commercialization of MOFtronics, 2D $c$-MOFs have exhibited extraordinary electrical conductivity up to $2500 \mathrm{~S} \mathrm{~cm}^{-1}$, band-like transport with mobility reaching $\sim 220 \mathrm{~cm}^{2} \mathrm{~V}^{-1} \mathrm{~s}^{-1}$ and tailorable band gaps that particularly encourages further studies. Furthermore, 2D $c$-MOFs feature many other unique properties of tunable porosity, redox and photoactivity, magnetic ordering and high stability, which make them appealing advanced electronic materials for the next-generation of electronic devices. It is expected that scalable production of 2D c-MOFs via controlled synthesis and facile processing will realize the MOFtronics in the near future.

\section{Conflicts of interest}

There are no conflicts of interest to declare.

\section{Acknowledgements}

We thank financial support from ERC Starting Grant (FC2DMOF, No. 852909), ERC Consolidator Grant (T2DCP), Coordination Networks: Building Blocks for Functional Systems (SPP 1928, COORNETs), European Union's Horizon 2020 research and innovation programme (GrapheneCore3, No. 881603), H2020-MSCAITN (ULTIMATE, No. 813036), DFG funding (CRC1415, No. 417590517) and the German Science Council, Center for Advancing Electronics Dresden, EXC1056.

\section{References}

1 H.-C. Zhou, J. R. Long and O. M. Yaghi, Chem. Rev., 2012, 112, 673.

2 J. Liu and C. Wöll, Chem. Soc. Rev., 2017, 46, 5730.

3 S. Krause, V. Bon, I. Senkovska, U. Stoeck, D. Wallacher, D. M. Többens, S. Zander, R. S. Pillai, G. Maurin, F.-X. Coudert and S. Kaskel, Nature, 2016, 532, 348.

4 S. Yuan, L. Feng, K. Wang, J. Pang, M. Bosch, C. Lollar, Y. Sun, J. Qin, X. Yang, P. Zhang, Q. Wang, L. Zou, Y. Zhang, L. Zhang, Y. Fang, J. Li and H.-C. Zhou, Adv. Mater., 2018, 30, 1704303.

5 L. Liu, L. Li, M. E. Ziebel and T. D. Harris, J. Am. Chem. Soc., 2020, 142, 4705.

6 S. Yuan, L. Huang, Z. Huang, D. Sun, J.-S. Qin, L. Feng, J. Li, X. Zou, T. Cagin and H.-C. Zhou, J. Am. Chem. Soc., 2020, 142, 4732.

7 S. Yuan, J.-S. Qin, J. Li, L. Huang, L. Feng, Y. Fang, C. Lollar, J. Pang, L. Zhang, D. Sun, A. Alsalme, T. Cagin and H.-C. Zhou, Nat. Commun., 2018, 9, 808.

8 H. Furukawa, K. E. Cordova, M. O’Keeffe and O. M. Yaghi, Science, 2013, 341, 1230444.

9 L. J. Murray, M. Dincă and J. R. Long, Chem. Soc. Rev., 2009, 38, 1294.

10 E. J. Kim, R. L. Siegelman, H. Z. H. Jiang, A. C. Forse, J.-H. Lee, J. D. Martell, P. J. Milner, J. M. Falkowski, J. B. Neaton, J. A. Reimer, S. C. Weston and J. R. Long, Science, 2020, 369, 392.

11 E. D. Bloch, W. L. Queen, R. Krishna, J. M. Zadrozny, C. M. Brown and J. R. Long, Science, 2012, 335, 1606.

12 Q. Yang, Q. Xu and H.-L. Jiang, Chem. Soc. Rev., 2017, 46, 4774.

13 L. Jiao, Y. Wang, H.-L. Jiang and Q. Xu, Adv. Mater., 2018, 30, 1703663.

14 H. Wang, Q.-L. Zhu, R. Zou and Q. Xu, Chem, 2017, 2, 52. 15 A. Schneemann, V. Bon, I. Schwedler, I. Senkovska, S. Kaskel and R. A. Fischer, Chem. Soc. Rev., 2014, 43, 6062.

16 L. E. Kreno, K. Leong, O. K. Farha, M. Allendorf, R. P. Van Duyne and J. T. Hupp, Chem. Rev., 2012, 112, 1105.

17 I. Stassen, N. Burtch, A. Talin, P. Falcaro, M. Allendorf and R. Ameloot, Chem. Soc. Rev., 2017, 46, 3185.

18 A. A. Talin, A. Centrone, A. C. Ford, M. E. Foster, V. Stavila, P. Haney, R. A. Kinney, V. Szalai, F. El Gabaly, H. P. Yoon, F. Léonard and M. D. Allendorf, Science, 2014, 343, 66.

19 M. Hmadeh, Z. Lu, Z. Liu, F. Gándara, H. Furukawa, S. Wan, V. Augustyn, R. Chang, L. Liao, F. Zhou, E. Perre, V. Ozolins, K. Suenaga, X. Duan, B. Dunn, Y. Yamamto, O. Terasaki and O. M. Yaghi, Chem. Mater., 2012, 24, 3511.

20 L. Sun, M. G. Campbell and M. Dincă, Angew. Chem., Int. Ed., 2016, 55, 3566.

21 R. Dong, T. Zhang and X. Feng, Chem. Rev., 2018, 118, 6189.

22 W.-H. Li, W.-H. Deng, G.-E. Wang and G. Xu, EnergyChem, 2020, 2, 100029.

23 M. Ko, L. Mendecki and K. A. Mirica, Chem. Commun., 2018, 54, 7873.

24 Z. Meng, R. M. Stolz, L. Mendecki and K. A. Mirica, Chem. Rev., 2019, 119, 478. 
25 L. S. Xie, G. Skorupskii and M. Dincă, Chem. Rev., 2020, 120, 8536.

26 W.-T. Koo, J.-S. Jang and I.-D. Kim, Chem, 2019, 5, 1938.

27 M. G. Campbell and M. Dincă, Sensors, 2017, 17.

28 M. G. Campbell, D. Sheberla, S. F. Liu, T. M. Swager and M. Dincă, Angew. Chem., Int. Ed., 2015, 54, 4349.

29 G. Wu, J. Huang, Y. Zang, J. He and G. Xu, J. Am. Chem. Soc., 2017, 139, 1360.

30 H. Arora, R. Dong, T. Venanzi, J. Zscharschuch, H. Schneider, M. Helm, X. Feng, E. Cánovas and A. Erbe, Adv. Mater., 2020, 32, 1907063.

31 X. Huang, P. Sheng, Z. Tu, F. Zhang, J. Wang, H. Geng, Y. Zou, C.-a. Di, Y. Yi, Y. Sun, W. Xu and D. Zhu, Nat. Commun., 2015, 6, 7408.

32 R. Dong, Z. Zhang, D. C. Tranca, S. Zhou, M. Wang, P. Adler, Z. Liao, F. Liu, Y. Sun, W. Shi, Z. Zhang, E. Zschech, S. C. B. Mannsfeld, C. Felser and X. Feng, Nat. Commun., 2018, 9, 2637.

33 C. Yang, R. Dong, M. Wang, P. S. Petkov, Z. Zhang, M. Wang, P. Han, M. Ballabio, S. A. Bräuninger, Z. Liao, J. Zhang, F. Schwotzer, E. Zschech, H.-H. Klauss, E. Cánovas, S. Kaskel, M. Bonn, S. Zhou, T. Heine and X. Feng, Nat. Commun., 2019, 10, 3260.

34 W. Li, L. Sun, J. Qi, P. Jarillo-Herrero, M. Dincă and J. Li, Chem. Sci., 2017, 8, 2859.

35 J. Park, M. Lee, D. Feng, Z. Huang, A. C. Hinckley, A. Yakovenko, X. Zou, Y. Cui and Z. Bao, J. Am. Chem. Soc., 2018, 140, 10315.

36 K. Wada, K. Sakaushi, S. Sasaki and H. Nishihara, Angew. Chem., Int. Ed., 2018, 57, 8886.

37 D. Sheberla, J. C. Bachman, J. S. Elias, C. J. Sun, Y. ShaoHorn and M. Dincă, Nat. Mater., 2017, 16, 220.

38 D. Feng, T. Lei, M. R. Lukatskaya, J. Park, Z. Huang, M. Lee, L. Shaw, S. Chen, A. A. Yakovenko, A. Kulkarni, J. Xiao, K. Fredrickson, J. B. Tok, X. Zou, Y. Cui and Z. Bao, Nat. Energy, 2018, 3, 30.

39 J. Park, A. C. Hinckley, Z. Huang, D. Feng, A. A. Yakovenko, M. Lee, S. Chen, X. Zou and Z. Bao, J. Am. Chem. Soc., 2018, 140, 14533.

40 J. H. Dou, L. Sun, Y. Ge, W. Li, C. H. Hendon, J. Li, S. Gul, J. Yano, E. A. Stach and M. Dincă, J. Am. Chem. Soc., 2017, 139, 13608.

41 X. Huang, H. Li, Z. Tu, L. Liu, X. Wu, J. Chen, Y. Liang, Y. Zou, Y. Yi, J. Sun, W. Xu and D. Zhu, J. Am. Chem. Soc., 2018, 140, 15153.

42 Y. Cui, J. Yan, Z. Chen, J. Zhang, Y. Zou, Y. Sun, W. Xu and D. Zhu, Adv. Sci., 2019, 6, 1802235.

43 Y. Jiang, I. Oh, S. H. Joo, O. Buyukcakir, X. Chen, S. H. Lee, M. Huang, W. K. Seong, S. K. Kwak, J.-W. Yoo and R. S. Ruoff, J. Am. Chem. Soc., 2019, 141, 16884.

44 L. Chen, J. Kim, T. Ishizuka, Y. Honsho, A. Saeki, S. Seki, H. Ihee and D. Jiang, J. Am. Chem. Soc., 2009, 131, 7287.

45 R. Dong, M. Pfeffermann, H. Liang, Z. Zheng, X. Zhu, J. Zhang and X. Feng, Angew. Chem., Int. Ed., 2015, 54, 12058.

46 Y. Cui, J. Yan, Z. Chen, W. Xing, C. Ye, X. Li, Y. Zou, Y. Sun, C. Liu, W. Xu and D. Zhu, iScience, 2020, 23, 100812.
47 Z. Meng and K. A. Mirica, Nano Res., 2021, 14, 369.

48 Q. Zhao, S. H. Li, R. L. Chai, X. Ren and C. Zhang, ACS Appl. Mater. Interfaces, 2020, 12, 7504.

49 H. Huang, Y. Zhao, Y. Bai, F. Li, Y. Zhang and Y. Chen, Adv. Sci., 2020, 7, 2000012.

50 H. Nagatomi, N. Yanai, T. Yamada, K. Shiraishi and N. Kimizuka, Chem. - Eur. J., 2018, 24, 1806.

51 Z. Meng, A. Aykanat and K. A. Mirica, J. Am. Chem. Soc., 2019, 141, 2046.

52 H. Zhong, K. H. Ly, M. Wang, Y. Krupskaya, X. Han, J. Zhang, J. Zhang, V. Kataev, B. Büchner, I. M. Weidinger, S. Kaskel, P. Liu, M. Chen, R. Dong and X. Feng, Angew. Chem., Int. Ed., 2019, 58, 10677.

53 H. Zhong, M. Ghorbani-Asl, K. H. Ly, J. Zhang, J. Ge, M. Wang, Z. Liao, D. Makarov, E. Zschech, E. Brunner, I. M. Weidinger, J. Zhang, A. V. Krasheninnikov, S. Kaskel, R. Dong and X. Feng, Nat. Commun., 2020, 11, 1721.

54 H. Jia, Y. Yao, J. Zhao, Y. Gao, Z. Luo and P. Du, J. Mater. Chem. A, 2018, 6, 1188.

55 M. Wang, H. Shi, P. Zhang, Z. Liao, M. Wang, H. Zhong, F. Schwotzer, A. S. Nia, E. Zschech, S. Zhou, S. Kaskel, R. Dong and X. Feng, Adv. Funct. Mater., 2020, 30, 2002664.

56 J. Liu, Y. Zhou, Z. Xie, Y. Li, Y. Liu, J. Sun, Y. Ma, O. Terasaki and L. Chen, Angew. Chem., Int. Ed., 2020, 59, 1081.

57 D. Sheberla, L. Sun, M. A. Blood-Forsythe, S. Er, C. R. Wade, C. K. Brozek, A. Aspuru-Guzik and M. Dincă, J. Am. Chem. Soc., 2014, 136, 8859.

58 T. Chen, J.-H. Dou, L. Yang, C. Sun, N. J. Libretto, G. Skorupskii, J. T. Miller and M. Dincă, J. Am. Chem. Soc., 2020, 142, 12367.

59 M. S. Yao, J. J. Zheng, A. Q. Wu, G. Xu, S. S. Nagarkar, G. Zhang, M. Tsujimoto, S. Sakaki, S. Horike, K. Otake and S. Kitagawa, Angew. Chem., Int. Ed., 2020, 59, 172.

60 V. Rubio-Giménez, S. Tatay and C. Martí-Gastaldo, Chem. Soc. Rev., 2020, 49, 5601.

61 R. Dong, Z. Zheng, D. C. Tranca, J. Zhang, N. Chandrasekhar, S. Liu, X. Zhuang, G. Seifert and X. Feng, Chem. - Eur. J., 2017, 23, 2255.

62 X. Sun, K.-H. Wu, R. Sakamoto, T. Kusamoto, H. Maeda, X. Ni, W. Jiang, F. Liu, S. Sasaki, H. Masunaga and H. Nishihara, Chem. Sci., 2017, 8, 8078.

63 X. Sun, K.-H. Wu, R. Sakamoto, T. Kusamoto, H. Maeda and H. Nishihara, Chem. Lett., 2017, 46, 1072.

64 J. Cui and Z. Xu, Chem. Commun., 2014, 50, 3986.

65 T. Pal, T. Kambe, T. Kusamoto, M. L. Foo, R. Matsuoka, R. Sakamoto and H. Nishihara, ChemPlusChem, 2015, 80, 1255.

66 T. Pal, S. Doi, H. Maeda, K. Wada, C. M. Tan, N. Fukui, R. Sakamoto, S. Tsuneyuki, S. Sasaki and H. Nishihara, Chem. Sci., 2019, 10, 5218.

67 Y. Misumi, A. Yamaguchi, Z. Zhang, T. Matsushita, N. Wada, M. Tsuchiizu and K. Awaga, J. Am. Chem. Soc., 2020, 142, 16513.

68 X. Huang, S. Zhang, L. Liu, L. Yu, G. Chen, W. Xu and D. Zhu, Angew. Chem., Int. Ed., 2018, 57, 146. 
69 N. T. T. Nguyen, H. Furukawa, F. Gándara, C. A. Trickett, H. M. Jeong, K. E. Cordova and O. M. Yaghi, J. Am. Chem. Soc., 2015, 137, 15394.

70 J. Huang, Y. He, M.-S. Yao, J. He, G. Xu, M. Zeller and Z. Xu, J. Mater. Chem. A, 2017, 5, 16139.

71 R. Matheu, E. Gutierrez-Puebla, M. Á. Monge, C. S. Diercks, J. Kang, M. S. Prévot, X. Pei, N. Hanikel, B. Zhang, P. Yang and O. M. Yaghi, J. Am. Chem. Soc., 2019, 141, 17081.

72 D. L. Turner, T. P. Vaid, P. W. Stephens, K. H. Stone, A. G. DiPasquale and A. L. Rheingold, J. Am. Chem. Soc., 2008, 130, 14.

73 X. Huang, Y. Qiu, Y. Wang, L. Liu, X. Wu, Y. Liang, Y. Cui, Y. Sun, Y. Zou, J. Zhu, W. Fang, J. Sun, W. Xu and D. Zhu, Angew. Chem., Int. Ed., 2020, 59, 2.

74 T. Kambe, R. Sakamoto, K. Hoshiko, K. Takada, M. Miyachi, J. H. Ryu, S. Sasaki, J. Kim, K. Nakazato, M. Takata and H. Nishihara, J. Am. Chem. Soc., 2013, 135, 2462.

75 T. Kambe, R. Sakamoto, T. Kusamoto, T. Pal, N. Fukui, K. Hoshiko, T. Shimojima, Z. Wang, T. Hirahara, K. Ishizaka, S. Hasegawa, F. Liu and H. Nishihara, J. Am. Chem. Soc., 2014, 136, 14357.

76 M. D. Allendorf, R. Dong, X. Feng, S. Kaskel, D. Matoga and V. Stavila, Chem. Rev., 2020, 120, 8581.

77 L.-P. Tang, L.-M. Tang, D. Wang, H.-X. Deng and K.-Q. Chen, J. Phys.: Condens. Matter, 2018, 30, 465301.

78 R. Dong, P. Han, H. Arora, M. Ballabio, M. Karakus, Z. Zhang, C. Shekhar, P. Adler, P. S. Petkov, A. Erbe, S. C. B. Mannsfeld, C. Felser, T. Heine, M. Bonn, X. Feng and E. Cánovas, Nat. Mater., 2018, 17, 1027.

79 A. J. Clough, J. M. Skelton, C. A. Downes, A. A. de la Rosa, J. W. Yoo, A. Walsh, B. C. Melot and S. C. Marinescu, J. Am. Chem. Soc., 2017, 139, 10863.

80 A. J. Clough, N. M. Orchanian, J. M. Skelton, A. J. Neer, S. A. Howard, C. A. Downes, L. F. J. Piper, A. Walsh, B. C. Melot and S. C. Marinescu, J. Am. Chem. Soc., 2019, 141, 16323.

81 D. Herebian, E. Bothe, F. Neese, T. Weyhermüller and K. Wieghardt, J. Am. Chem. Soc., 2003, 125, 9116.

82 X. Chen, Y. Hu, D. Wu, L. Weng and B. Kang, Polyhedron, 1991, 10, 2651.

83 A. Das, Z. Han, W. W. Brennessel, P. L. Holland and R. Eisenberg, ACS Catal., 2015, 5, 1397.

84 J. Liu and Q. Sun, ChemPhysChem, 2015, 16, 614.

85 M. Zhao, A. Wang and X. Zhang, Nanoscale, 2013, 5, 10404. 86 M. A. Springer, T.-J. Liu, A. Kuc and T. Heine, Chem. Soc. Rev., 2020, 49, 2007.

87 Q. Jiang, P. Xiong, J. Liu, Z. Xie, Q. Wang, X. Q. Yang, E. Hu, Y. Cao, J. Sun, Y. Xu and L. Chen, Angew. Chem., Int. Ed., 2020, 59, 5273.

88 Z. Wang, G. Wang, H. Qi, M. Wang, M. Wang, S. Park, H. Wang, M. Yu, U. Kaiser, A. Fery, S. Zhou, R. Dong and X. Feng, Chem. Sci., 2020, 11, 7665.

89 K. W. Nam, S. S. Park, R. dos Reis, V. P. Dravid, H. Kim, C. A. Mirkin and J. F. Stoddart, Nat. Commun., 2019, 10, 4948.
90 W.-H. Li, K. Ding, H.-R. Tian, M.-S. Yao, B. Nath, W.-H. Deng, Y. Wang and G. Xu, Adv. Funct. Mater., 2017, 27, 1702067.

91 M. G. Campbell, S. F. Liu, T. M. Swager and M. Dincă, J. Am. Chem. Soc., 2015, 137, 13780.

92 M. Ko, A. Aykanat, M. K. Smith and K. A. Mirica, Sensors, 2017, 17.

93 B. Hoppe, K. D. J. Hindricks, D. P. Warwas, H. A. Schulze, A. Mohmeyer, T. J. Pinkvos, S. Zailskas, M. R. Krey, C. Belke, S. König, M. Fröba, R. J. Haug and P. Behrens, CrystEngComm, 2018, 20, 6458.

94 A. Mähringer, A. C. Jakowetz, J. M. Rotter, B. J. Bohn, J. K. Stolarczyk, J. Feldmann, T. Bein and D. D. Medina, ACS Nano, 2019, 13, 6711.

95 R. W. Day, D. K. Bediako, M. Rezaee, L. R. Parent, G. Skorupskii, M. Q. Arguilla, C. H. Hendon, I. Stassen, N. C. Gianneschi, P. Kim and M. Dincă, ACS Cent. Sci., 2019, 5, 1959.

96 M.-S. Yao, X.-J. Lv, Z.-H. Fu, W.-H. Li, W.-H. Deng, G.-D. Wu and G. Xu, Angew. Chem., Int. Ed., 2017, 56, 16510.

97 X. Song, X. Wang, Y. Li, C. Zheng, B. Zhang, C.-a. Di, F. Li, C. Jin, W. Mi, L. Chen and W. Hu, Angew. Chem., Int. Ed., 2020, 59, 1118.

98 V. Rubio-Giménez, M. Galbiati, J. Castells-Gil, N. AlmoraBarrios, J. Navarro-Sánchez, G. Escorcia-Ariza, M. Mattera, T. Arnold, J. Rawle, S. Tatay, E. Coronado and C. MartíGastaldo, Adv. Mater., 2018, 30, 1704291.

99 E. M. Miner, L. Wang and M. Dincă, Chem. Sci., 2018, 9, 6286.

100 H. Wu, W. Zhang, S. Kandambeth, O. Shekhah, M. Eddaoudi and H. N. Alshareef, Adv. Energy Mater., 2019, 9, 1900482.

101 S. Zhou, X. Kong, B. Zheng, F. Huo, M. Strømme and C. Xu, ACS Nano, 2019, 13, 9578.

102 M. K. Smith and K. A. Mirica, J. Am. Chem. Soc., 2017, 139, 16759.

103 Y. Lian, W. Yang, C. Zhang, H. Sun, Z. Deng, W. Xu, L. Song, Z. Ouyang, Z. Wang, J. Guo and Y. Peng, Angew. Chem., Int. Ed., 2020, 59, 286.

104 Y. Zang, F. Pei, J. Huang, Z. Fu, G. Xu and X. Fang, Adv. Energy Mater., 2018, 8, 1802052.

105 L. Sun, B. Liao, D. Sheberla, D. Kraemer, J. Zhou, E. A. Stach, D. Zakharov, V. Stavila, A. A. Talin, Y. Ge, M. D. Allendorf, G. Chen, F. Léonard and M. Dincă, Joule, 2017, 1, 168.

106 K. Yuan, T. Song, X. Zhu, B. Li, B. Han, L. Zheng, J. Li, X. Zhang and W. Hu, Small, 2019, 15, 1804845.

107 B. Wang, Y. Luo, B. Liu and G. Duan, ACS Appl. Mater. Interfaces, 2019, 11, 35935.

108 X. Huang, H. Yao, Y. Cui, W. Hao, J. Zhu, W. Xu and D. Zhu, ACS Appl. Mater. Interfaces, 2017, 9, 40752.

109 L. Mendecki, M. Ko, X. Zhang, Z. Meng and K. A. Mirica, J. Am. Chem. Soc., 2017, 139, 17229.

110 Z. Chen, Y. Cui, Y. Jin, L. Liu, J. Yan, Y. Sun, Y. Zou, Y. Sun, W. Xu and D. Zhu, J. Mater. Chem. C, 2020, 8, 8199.

111 Z. Chen, Y. Cui, C. Ye, L. Liu, X. Wu, Y. Sun, W. Xu and D. Zhu, Chem. - Eur. J., 2020, 26, 12868. 
112 Z. F. Wang, N. Su and F. Liu, Nano Lett., 2013, 13, 2842.

113 S. Chen, J. Dai and X. C. Zeng, Phys. Chem. Chem. Phys., 2015, 17, 5954.

114 L. Sun, C. H. Hendon, S. S. Park, Y. Tulchinsky, R. Wan, F. Wang, A. Walsh and M. Dincă, Chem. Sci., 2017, 8, 4450.

115 M. L. Aubrey, B. M. Wiers, S. C. Andrews, T. Sakurai, S. E. Reyes-Lillo, S. M. Hamed, C.-J. Yu, L. E. Darago, J. A. Mason, J.-O. Baeg, F. Grandjean, G. J. Long, S. Seki, J. B. Neaton, P. Yang and J. R. Long, Nat. Mater., 2018, 17, 625 .

116 J. G. Park, M. L. Aubrey, J. Oktawiec, K. Chakarawet, L. E. Darago, F. Grandjean, G. J. Long and J. R. Long, J. Am. Chem. Soc., 2018, 140, 8526.

117 X. Wu, Y. Qiu, Z. Chen, B. Guan, X. Hao, A. I. Rykov, Y. Sun, L. Liu, Y. Zou, J. Sun, W. Xu and D. Zhu, Angew. Chem., Int. Ed., 2020, 59, 1.

118 A. J. Clough, J. W. Yoo, M. H. Mecklenburg and S. C. Marinescu, J. Am. Chem. Soc., 2015, 137, 118.

119 A. C. Hinckley, J. Park, J. Gomes, E. Carlson and Z. Bao, J. Am. Chem. Soc., 2020, 142, 11123.

120 L.-P. Tang, L.-M. Tang, H. Geng, Y.-P. Yi, Z. Wei, K.-Q. Chen and H.-X. Deng, Appl. Phys. Lett., 2018, 112, 012101.

121 G. Skorupskii, B. A. Trump, T. W. Kasel, C. M. Brown, C. H. Hendon and M. Dincă, Nat. Chem., 2020, 12, 131.

122 V. Rubio-Gimenez, N. Almora-Barrios, G. Escorcia-Ariza, M. Galbiati, M. Sessolo, S. Tatay and C. Marti-Gastaldo, Angew. Chem., Int. Ed., 2018, 57, 15086.

123 L. Dong, Y. Kim, D. Er, A. M. Rappe and V. B. Shenoy, Phys. Rev. Lett., 2016, 116, 096601.

124 M. E. Foster, K. Sohlberg, C. D. Spataru and M. D. Allendorf, J. Phys. Chem. C, 2016, 120, 15001.

125 M. E. Foster, K. Sohlberg, M. D. Allendorf and A. A. Talin, J. Phys. Chem. Lett., 2018, 9, 481.

126 X. Zhang, Y. Zhou, B. Cui, M. Zhao and F. Liu, Nano Lett., 2017, 17, 6166.

127 Y. Jiang, I. Oh, S. H. Joo, Y.-S. Seo, S. H. Lee, W. K. Seong, Y. J. Kim, J. Hwang, S. K. Kwak, J.-W. Yoo and R. S. Ruoff, J. Am. Chem. Soc., 2020, 142, 18346.

128 B. Lukose, A. Kuc and T. Heine, Chem. - Eur. J., 2011, 17, 2388.

129 A. Kuc, M. A. Springer, K. Batra, R. Juarez-Mosqueda, C. Wöll and T. Heine, Adv. Funct. Mater., 2020, 30, 1908004.

130 A. P. Côté, A. I. Benin, N. W. Ockwig, M. O'Keeffe, A. J. Matzger and O. M. Yaghi, Science, 2005, 310, 1166.

131 M. J. Kory, M. Wörle, T. Weber, P. Payamyar, S. W. van de Poll, J. Dshemuchadse, N. Trapp and A. D. Schlüter, Nat. Chem., 2014, 6, 779.

132 T. Ma, E. A. Kapustin, S. X. Yin, L. Liang, Z. Zhou, J. Niu, L.H. Li, Y. Wang, J. Su, J. Li, X. Wang, W. D. Wang, W. Wang, J. Sun and O. M. Yaghi, Science, 2018, 361, 48.

133 A. M. Evans, L. R. Parent, N. C. Flanders, R. P. Bisbey, E. Vitaku, M. S. Kirschner, R. D. Schaller, L. X. Chen, N. C. Gianneschi and W. R. Dichtel, Science, 2018, 361, 52.

134 J. Ogle, N. Lahiri, C. Jaye, C. J. Tassone, D. A. Fischer, J. Louie and L. Whittaker-Brooks, Adv. Funct. Mater., 2020, 2006920, DOI: 10.1002/adfm.202006920.
135 N. Lahiri, N. Lotfizadeh, R. Tsuchikawa, V. V. Deshpande and J. Louie, J. Am. Chem. Soc., 2017, 139, 19.

136 J. Sakamoto, J. van Heijst, O. Lukin and A. D. Schlüter, Angew. Chem., Int. Ed., 2009, 48, 1030.

137 L. Wang, H. Sahabudeen, T. Zhang and R. Dong, npj 2D Mater. Appl., 2018, 2, 26.

138 T. Zhang, H. Qi, Z. Liao, Y. D. Horev, L. A. Panes-Ruiz, P. S. Petkov, Z. Zhang, R. Shivhare, P. Zhang, K. Liu, V. Bezugly, S. Liu, Z. Zheng, S. Mannsfeld, T. Heine, G. Cuniberti, H. Haick, E. Zschech, U. Kaiser, R. Dong and X. Feng, Nat. Commun., 2019, 10, 4225.

139 K. Liu, L. Wang and R. Dong, J. Mater. Chem. C, 2020, 8, 10696.

140 K. Liu, H. Qi, R. Dong, R. Shivhare, M. Addicoat, T. Zhang, H. Sahabudeen, T. Heine, S. Mannsfeld, U. Kaiser, Z. Zheng and X. Feng, Nat. Chem., 2019, 11, 994.

141 X. Zhuang, Y. Mai, D. Wu, F. Zhang and X. Feng, Adv. Mater., 2015, 27, 403.

142 X. Feng and A. D. Schlüter, Angew. Chem., Int. Ed., 2018, 57, 13748.

143 S. Liu, Y. C. Wang, C. M. Chang, T. Yasuda, N. Fukui, H. Maeda, P. Long, K. Nakazato, W. B. Jian, W. Xie, K. Tsukagoshi and H. Nishihara, Nanoscale, 2020, 12, 6983.

144 A. Ciesielski and P. Samorì, Chem. Soc. Rev., 2014, 43, 381. 145 V. Nicolosi, M. Chhowalla, M. G. Kanatzidis, M. S. Strano and J. N. Coleman, Science, 2013, 340, 1226419.

146 R. Hou, M. Miao, Q. Wang, T. Yue, H. Liu, H. S. Park, K. Qi and B. Y. Xia, Adv. Energy Mater., 2020, 10, 1901892.

147 X. Du, J. Zhang, H. Wang, Z. Huang, A. Guo, L. Zhao, Y. Niu, X. Li, B. Wu and Y. Liu, Mater. Chem. Front., 2020, 4, 243.

148 M. Wang, M. Ballabio, M. Wang, H.-H. Lin, B. P. Biswal, X. Han, S. Paasch, E. Brunner, P. Liu, M. Chen, M. Bonn, T. Heine, S. Zhou, E. Cánovas, R. Dong and X. Feng, J. Am. Chem. Soc., 2019, 141, 16810.

149 B. P. Biswal, S. Valligatla, M. Wang, T. Banerjee, N. A. Saad, B. M. K. Mariserla, N. Chandrasekhar, D. Becker, M. Addicoat, I. Senkovska, R. Berger, D. N. Rao, S. Kaskel and X. Feng, Angew. Chem., Int. Ed., 2019, 58, 6896.

150 S. Xu, G. Wang, B. P. Biswal, M. Addicoat, S. Paasch, W. Sheng, X. Zhuang, E. Brunner, T. Heine, R. Berger and X. Feng, Angew. Chem., Int. Ed., 2019, 58, 849.

151 L. E. Darago, M. L. Aubrey, C. J. Yu, M. I. Gonzalez and J. R. Long, J. Am. Chem. Soc., 2015, 137, 15703.

152 R. Sakamoto, K. Takada, T. Pal, H. Maeda, T. Kambe and H. Nishihara, Chem. Commun., 2017, 53, 5781.

153 Q. Jiang, C. Zhou, H. Meng, Y. Han, X. Shi, C. Zhan and R. Zhang, J. Mater. Chem. A, 2020, 8, 15271.

154 K. Zhao, W. Zhu, S. Liu, X. Wei, G. Ye, Y. Su and Z. He, Nanoscale Adv., 2020, 2, 536.

155 A.-Q. Wu, W.-Q. Wang, H.-B. Zhan, L.-A. Cao, X.-L. Ye, J.-J. Zheng, P. N. Kumar, K. Chiranjeevulu, W.-H. Deng, G.-E. Wang, M.-S. Yao and G. Xu, Nano Res., 2021, 14, 438.

156 L.-A. Cao, M.-S. Yao, H.-J. Jiang, S. Kitagawa, X.-L. Ye, W.-H. Li and G. Xu, J. Mater. Chem. A, 2020, 8, 9085.

157 G. Iannaccone, F. Bonaccorso, L. Colombo and G. Fiori, Nat. Nanotechnol., 2018, 13, 183. 
158 E. Pomerantseva and Y. Gogotsi, Nat. Energy, 2017, 2, 17089.

159 G. Fiori, F. Bonaccorso, G. Iannaccone, T. Palacios, D. Neumaier, A. Seabaugh, S. K. Banerjee and L. Colombo, Nat. Nanotechnol., 2014, 9, 768.

160 M. Zhang, B.-H. Zheng, J. Xu, N. Pan, J. Yu, M. Chen and H. Cao, Chem. Commun., 2018, 54, 13579.

161 R. Makiura, S. Motoyama, Y. Umemura, H. Yamanaka, O. Sakata and H. Kitagawa, Nat. Mater., 2010, 9, 565.

162 Z. A. Gao, C.-H. Hsu, J. Liu, F.-C. Chuang, R. Zhang, B. Xia, H. Xu, L. Huang, Q. Jin, P. N. Liu and N. Lin, Nanoscale, 2019, 11, 878.

163 M. Lischka, R. Dong, M. Wang, N. Martsinovich, M. Fritton, L. Grossmann, W. M. Heckl, X. Feng and M. Lackinger, Chem. - Eur. J., 2019, 25, 1975.

164 X. Meng, E. Kolodzeiski, X. Huang, A. Timmer, B. Schulze Lammers, H.-Y. Gao, H. Mönig, L. Liu, W. Xu, S. Amirjalayer, D. Zhu and H. Fuchs, ChemNanoMat, 2020, 6, 1.

165 R. Zhang, J. Liu, Y. Gao, M. Hua, B. Xia, P. Knecht, A. C. Papageorgiou, J. Reichert, J. V. Barth, H. Xu, L. Huang and N. Lin, Angew. Chem., Int. Ed., 2020, 59, 2669.

166 H. Qi, H. Sahabudeen, B. Liang, M. Položij, M. A. Addicoat, T. E. Gorelik, M. Hambsch, M. Mundszinger, S. Park, B. V. Lotsch, S. C. B. Mannsfeld, Z. Zheng, R. Dong, T. Heine, X. Feng and U. Kaiser, Sci. Adv., 2020, 6, eabb5976.

167 Y. Peng, Y. Li, Y. Ban, H. Jin, W. Jiao, X. Liu and W. Yang, Science, 2014, 346, 1356.

168 I.-Y. Jeon, Y.-R. Shin, G.-J. Sohn, H.-J. Choi, S.-Y. Bae, J. Mahmood, S.-M. Jung, J.-M. Seo, M.-J. Kim, D. Wook Chang, L. Dai and J.-B. Baek, Proc. Natl. Acad. Sci. U. S. A., 2012, 109, 5588.

169 P.-Z. Li, Y. Maeda and Q. Xu, Chem. Commun., 2011, 47, 8436.

170 S. Yang, P. Zhang, F. Wang, A. G. Ricciardulli, M. R. Lohe, P. W. M. Blom and X. Feng, Angew. Chem., Int. Ed., 2018, 57, 15491.

171 Z. Zhang, S. Yang, P. Zhang, J. Zhang, G. Chen and X. Feng, Nat. Commun., 2019, 10, 2920.

172 M. Zhao, Y. Huang, Y. Peng, Z. Huang, Q. Ma and H. Zhang, Chem. Soc. Rev., 2018, 47, 6267.

173 H. Shi, M. Li, A. Shaygan Nia, M. Wang, S. Park, Z. Zhang, M. R. Lohe, S. Yang and X. Feng, Adv. Mater., 2020, 32, 1907244.

174 S. C. Junggeburth, L. Diehl, S. Werner, V. Duppel, W. Sigle and B. V. Lotsch, J. Am. Chem. Soc., 2013, 135, 6157.

175 M. Zhao, Y. Wang, Q. Ma, Y. Huang, X. Zhang, J. Ping, Z. Zhang, Q. Lu, Y. Yu, H. Xu, Y. Zhao and H. Zhang, Adv. Mater., 2015, 27, 7372.

176 M. K. Smith, K. E. Jensen, P. A. Pivak and K. A. Mirica, Chem. Mater., 2016, 28, 5264.

177 L. Mendecki and K. A. Mirica, ACS Appl. Mater. Interfaces, 2018, 10, 19248.

178 I. Stassen, J. H. Dou, C. Hendon and M. Dincă, ACS Cent. Sci., 2019, 5, 1425.
179 Z. Jin, J. Yan, X. Huang, W. Xu, S. Yang, D. Zhu and J. Wang, Nano Energy, 2017, 40, 376.

180 S. Datta and B. Das, Appl. Phys. Lett., 1990, 56, 665.

181 J. Wunderlich, B.-G. Park, A. C. Irvine, L. P. Zârbo, E. Rozkotová, P. Nemec, V. Novák, J. Sinova and T. Jungwirth, Science, 2010, 330, 1801.

182 X. Sun, S. Vélez, A. Atxabal, A. Bedoya-Pinto, S. Parui, X. Zhu, R. Llopis, F. Casanova and L. E. Hueso, Science, 2017, 357, 677.

183 V. A. Dediu, L. E. Hueso, I. Bergenti and C. Taliani, Nat. Mater., 2009, 8, 707.

184 L. Yang, X. He and M. Dincă, J. Am. Chem. Soc., 2019, 141, 10475. 185 I.-R. Jeon, B. Negru, R. P. Van Duyne and T. D. Harris, J. Am. Chem. Soc., 2015, 137, 15699.

186 J. A. DeGayner, I.-R. Jeon, L. Sun, M. Dincă and T. D. Harris, J. Am. Chem. Soc., 2017, 139, 4175.

187 K. S. Pedersen, P. Perlepe, M. L. Aubrey, D. N. Woodruff, S. E. Reyes-Lillo, A. Reinholdt, L. Voigt, Z. Li, K. Borup, M. Rouzières, D. Samohvalov, F. Wilhelm, A. Rogalev, J. B. Neaton, J. R. Long and R. Clérac, Nat. Chem., 2018, 10, 1056.

188 A. E. Thorarinsdottir and T. D. Harris, Chem. Rev., 2020, 120, 8716.

189 X. Li, X. Li and J. Yang, J. Phys. Chem. Lett., 2020, 11, 4193.

190 D. Sun, E. Ehrenfreund and Z. Valy Vardeny, Chem. Commun., 2014, 50, 1781.

191 Y. Shi, M. R. Momeni, Y.-J. Chen, Z. Zhang and F. A. Shakib, Chem. Mater., 2020, 32, 9664.

192 J. Sun, L. Guo, X. Sun, J. Zhang, Y. Liu, L. Hou and C. Yuan, J. Mater. Chem. A, 2019, 7, 24788.

193 S. Gu, Z. Bai, S. Majumder, B. Huang and G. Chen, J. Power Sources, 2019, 429, 22.

194 W. Zhou, S. Lv, X. Liu, Y. Li and J. Liu, Chem. Commun., 2019, 55, 11207.

195 A. Nazir, H. T. T. Le, C. W. Min, A. Kasbe, J. Kim, C. S. Jin and C. J. Park, Nanoscale, 2020, 12, 1629.

196 F. Xu, Y. Zhai, E. Zhang, Q. Liu, G. Jiang, X. Xu, Y. Qiu, X. Liu, H. Wang and S. Kaskel, Angew. Chem., Int. Ed., 2020, 59, 19460.

197 M. Yu, R. Dong and X. Feng, J. Am. Chem. Soc., 2020, 142, 12903.

198 J. Liu, X. Song, T. Zhang, S. Liu, H. Wen and L. Chen, Angew. Chem., Int. Ed., 2020, 59, 2.

199 X. Li, X. Yang, H. Xue, H. Pang and Q. Xu, EnergyChem, 2020, 2, 100027.

200 K. Wada, H. Maeda, T. Tsuji, K. Sakaushi, S. Sasaki and H. Nishihara, Inorg. Chem., 2020, 59, 10604.

201 B. Xu, H. Zhang, H. Mei and D. Sun, Coord. Chem. Rev., 2020, 420, 213438.

202 Z. Wu, D. Adekoya, X. Huang, M. J. Kiefel, J. Xie, W. Xu, Q. Zhang, D. Zhu and S. Zhang, ACS Nano, 2020, 14, 12016.

203 F. Wang, Z. Liu, C. Yang, H. Zhong, G. Nam, P. Zhang, R. Dong, Y. Wu, J. Cho, J. Zhang and X. Feng, Adv. Mater., 2020, 32, 1905361.

204 P. Zhang, F. Wang, M. Yu, X. Zhuang and X. Feng, Chem. Soc. Rev., 2018, 47, 7426. 
205 S. Bi, H. Banda, M. Chen, L. Niu, M. Chen, T. Wu, J. Wang, R. Wang, J. Feng, T. Chen, M. Dincă, A. A. Kornyshev and G. Feng, Nat. Mater., 2020, 19, 552.

206 M. Yu, H. Shao, G. Wang, F. Yang, C. Liang, P. Rozier, C.Z. Wang, X. Lu, P. Simon and X. Feng, Nat. Commun., 2020, 11, 1348.

207 M. Yu, D. Lin, H. Feng, Y. Zeng, Y. Tong and X. Lu, Angew. Chem., Int. Ed., 2017, 56, 5454.

208 K. Jiang, I. A. Baburin, P. Han, C. Yang, X. Fu, Y. Yao, J. Li, E. Cánovas, G. Seifert, J. Chen, M. Bonn, X. Feng and X. Zhuang, Adv. Funct. Mater., 2020, 30, 1908243.

209 P. Zhang, Y. Li, G. Wang, F. Wang, S. Yang, F. Zhu, X. Zhuang, O. G. Schmidt and X. Feng, Adv. Mater., 2019, 31, 1806005.

210 C. Yang, K. S. Schellhammer, F. Ortmann, S. Sun, R. Dong, M. Karakus, Z. Mics, M. Löffler, F. Zhang, X. Zhuang, E. Cánovas, G. Cuniberti, M. Bonn and X. Feng, Angew. Chem., Int. Ed., 2017, 56, 3920.

211 Y. He, S. Yang, Y. Fu, F. Wang, J. Ma, G. Wang, G. Chen, M. Wang, R. Dong, P. Zhang and X. Feng, Small Struct., 2020, 2000095, DOI: 10.1002/sstr.202000095.

212 Y. Zhong, B. Cheng, C. Park, A. Ray, S. Brown, F. Mujid, J.U. Lee, H. Zhou, J. Suh, K.-H. Lee, A. J. Mannix, K. Kang, S. J. Sibener, D. A. Muller and J. Park, Science, 2019, 366, 1379.

213 F. Bonaccorso, L. Colombo, G. Yu, M. Stoller, V. Tozzini, A. C. Ferrari, R. S. Ruoff and V. Pellegrini, Science, 2015, 347, 1246501.

214 G. Wang, J. Zhang, S. Yang, F. Wang, X. Zhuang, K. Müllen and X. Feng, Adv. Energy Mater., 2018, 8, 1702254.

215 H. Peng, J. Raya, F. Richard, W. Baaziz, O. Ersen, A. Ciesielski and P. Samorì, Angew. Chem., Int. Ed., 2020, 59, 2.

216 M. Yu, N. Chandrasekhar, R. K. M. Raghupathy, K. H. Ly, H. Zhang, E. Dmitrieva, C. Liang, X. Lu, T. D. Kühne, H. Mirhosseini, I. M. Weidinger and X. Feng, J. Am. Chem. Soc., 2020, 142, 19570.

217 Q. Ma, P. Yin, M. Zhao, Z. Luo, Y. Huang, Q. He, Y. Yu, Z. Liu, Z. Hu, B. Chen and H. Zhang, Adv. Mater., 2019, 31, 1808249.

218 J. Zhang, Z. Zhao, Z. Xia and L. Dai, Nat. Nanotechnol., 2015, 10, 444.

219 L. Dai, Y. Xue, L. Qu, H.-J. Choi and J.-B. Baek, Chem. Rev., 2015, 115, 4823.

220 G. Chen, J. Zhang, F. Wang, L. Wang, Z. Liao, E. Zschech, K. Müllen and X. Feng, Chem. - Eur. J., 2018, 24, 18413.

221 J. Zhang, G. Chen, K. Müllen and X. Feng, Adv. Mater., 2018, 30, 1800528.

222 G. Chen, T. Wang, J. Zhang, P. Liu, H. Sun, X. Zhuang, M. Chen and X. Feng, Adv. Mater., 2018, 30, 1706279.

223 H. Wang, X.-B. Li, L. Gao, H.-L. Wu, J. Yang, L. Cai, T.B. Ma, C.-H. Tung, L.-Z. Wu and G. Yu, Angew. Chem., Int. Ed., 2018, 57, 192.
224 M. Yu, Z. Wang, C. Hou, Z. Wang, C. Liang, C. Zhao, Y. Tong, X. Lu and S. Yang, Adv. Mater., 2017, 29, 1602868.

225 H. Zhong, J. Wang, F. Meng and X. Zhang, Angew. Chem., Int. Ed., 2016, 55, 9937.

226 F. Wei, T. Wang, X. Jiang, Y. Ai, A. Cui, J. Cui, J. Fu, J. Cheng, L. Lei, Y. Hou and S. Liu, Adv. Funct. Mater., 2020, 30, 2002092.

227 D. Xing, Y. Wang, P. Zhou, Y. Liu, Z. Wang, P. Wang, Z. Zheng, H. Cheng, Y. Dai and B. Huang, Appl. Catal., B, 2020, 278, 119295.

228 W. Xiong, X. Cheng, T. Wang, Y. Luo, J. Feng, S. Lu, A. M. Asiri, W. Li, Z. Jiang and X. Sun, Nano Res., 2020, 13, 1008.

229 W. Sheng, I. Amin, C. Neumann, R. Dong, T. Zhang, E. Wegener, W.-L. Chen, P. Förster, H. Q. Tran, M. Löffler, A. Winter, R. D. Rodriguez, E. Zschech, C. K. Ober, X. Feng, A. Turchanin and R. Jordan, Small, 2019, 15, 1805228.

230 C. A. Downes, A. J. Clough, K. Chen, J. W. Yoo and S. C. Marinescu, ACS Appl. Mater. Interfaces, 2018, 10, 1719.

231 J. Wang, J. Wang, X. Song, S. Qi and M. Zhao, Appl. Surf. Sci., 2020, 511, 145393.

232 W.-H. Li, J. Lv, Q. Li, J. Xie, N. Ogiwara, Y. Huang, H. Jiang, H. Kitagawa, G. Xu and Y. Wang, J. Mater. Chem. A, 2019, 7, 10431.

233 C. Li, L. Shi, L. Zhang, P. Chen, J. Zhu, X. Wang and Y. Fu, J. Mater. Chem. A, 2020, 8, 369.

234 E. M. Miner, T. Fukushima, D. Sheberla, L. Sun, Y. Surendranath and M. Dincă, Nat. Commun., 2016, 7, 10942.

235 E. M. Miner, S. Gul, N. D. Ricke, E. Pastor, J. Yano, V. K. Yachandra, T. Van Voorhis and M. Dincă, ACS Catal., 2017, 7, 7726.

236 H. Yoon, S. Lee, S. Oh, H. Park, S. Choi and M. Oh, Small, 2019, 15, 1805232.

237 X.-H. Liu, W.-L. Hu, W.-J. Jiang, Y.-W. Yang, S. Niu, B. Sun, J. Wu and J.-S. Hu, ACS Appl. Mater. Interfaces, 2017, 9, 28473.

238 S. S. Shinde, C. H. Lee, J.-Y. Jung, N. K. Wagh, S.-H. Kim, D.-H. Kim, C. Lin, S. U. Lee and J.-H. Lee, Energy Environ. Sci., 2019, 12, 727.

239 F. Wu, W. Fang, X. Yang, J. Xu, J. Xia and Z. Wang, J. Chin. Chem. Soc., 2019, 66, 522.

240 M. Ko, L. Mendecki, A. M. Eagleton, C. G. Durbin, R. M. Stolz, Z. Meng and K. A. Mirica, J. Am. Chem. Soc., 2020, 142, 11717.

241 J.-H. Dou, M. Q. Arguilla, Y. Luo, J. Li, W. Zhang, L. Sun, J. L. Mancuso, L. Yang, T. Chen, L. R. Parent, G. Skorupskii, N. J. Libretto, C. Sun, M. C. Yang, P. V. Dip, E. J. Brignole, J. T. Miller, J. Kong, C. H. Hendon, J. Sun and M. Dincă, Nat. Mater., 2020, DOI: 10.1038/s41563-020-00847-7. 